\author{
UNIVERSIDADE DE SÃO PAULO \\ ESCOLA DE ENGENHARIA DE SÃO CARLOS \\ DEPARTAMENTO DE ENGENHARIA DE ESTRUTURAS
}

EDWIN ALEXANDER MORANTES RODRÍGUEZ

DESENVOLVIMENTO DE MODELO DE LIGAÇÃO DESLIZANTE PARA A SIMULAÇÃO DE DISPOSITIVOS DE CONTROLE DE VIBRAÇÃO NA ANÁLISE NÃO LINEAR GEOMÉTRICA DE ESTRUTURAS

SÃO CARLOS 



\section{DESENVOLVIMENTO DE MODELO DE LIGAÇÃO DESLIZANTE PARA A SIMULAÇÃO DE DISPOSITIVOS DE CONTROLE DE VIBRAÇÃO NA ANÁLISE NÃO LINEAR GEOMÉTRICA DE ESTRUTURAS}

\section{VERSÃO CORRIGIDA}

A versão original encontra-se na Escola de Engenharia de São Carlos

Dissertação apresentada ao Programa de Pós Graduação em Engenharia de Estruturas da Escola de Engenharia de São Carlos, como parte dos requisitos para obtenção do título de Mestre em Ciências, Programa: Engenharia Civil (Estruturas).

Áreas de concentração: Estruturas

Orientador: Prof. Tit. Humberto Breves Coda

SÃO CARLOS 
AUTORIZO A REPRODUÇÃO TOTAL OU PARCIAL DESTE TRABALHO, POR QUALQUER MEIO CONVENCIONAL OU ELETRONICO, PARA FINS DE ESTUDO E PESQUISA, DESDE QUE CITADA A FONTE.

Morantes Rodriguez, Edwin Alexander

Desenvolvimento de modelo de ligaçäo deslizante

para a simulaçäo de dispositivos de controle de

vibração na analise nâo linear geometrica de estruturas

/ Edrin Alexander Morantes Rodriguer; orientador

Humberto Breves Coda. Bão Carlos, 2017.

Dissertação (Mestrado) - Programa de Pós-Graduação em Engenharia Civil (Engenharia de Estruturas) e Area de Concentração em Estruturas -- Escola de Engenharia de são Carlos da Universidade de 3ăo Paulo, 2017.

1. Nétodo dos elementos finitos posicional. 2. Analise não linear geometrica de estruturas. 3 . Ligações deslizantes. 4. Dinâmica das estruturas e mecanismos. 5. Controle de vibração. I. Titulo. 


\section{FOLHA DE JULGAMENTO}

Candidato: Engenheiro EDWIN ALEXANDER MORANIES RODRIGUEZ.

Título da dissertação: "Desenvolvimento de modelo de ligação deslizante para a simulação de dispositivos de controle de vibração na análise não linear geométrica de estruturas ".

Data da defesa: 03/04/2017.

Comissão Julgadora:

Resultado:

Prof. Titular Humberto Breves Coda (Orientador)

(Escola de Engenharia de São Carlos/EESC)

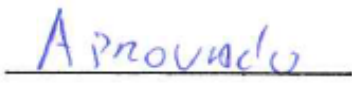

Prof. Dr. Leopoldo Pisanelli Rodrigues de Oliveira

(Escola de Engenharia de São Carlos/EESC)

Prof. Dr. José Benaque Rubert

APROVADO

(Universidade Federal de São Carlos/UFSCar)

Coordenador do Programa de Pós-Graduação em Engenheira Civil (Engenharia de Estruturas):

Prof. Titular Humberto Breves Coda

Presidente da Comissão de Pós-Graduação:

Prof. Associado Luís Fernando Cosła Alberło 

Para minha família:

Rafael, Marina, Claudia,

Shirley e Mauricio. 



\section{AGRADECIMENTOS}

Em primeiro lugar gostaria de agradecer a Deus pelas grandes oportunidades que tem me dado ao longo da minha vida.

Agradecer à minha família, Rafael, Marina, Claudia, Shirley e Mauricio, pelo suporte, pelos grandes sacrifícios e pelos sábios conselhos que formam a base do meu ser.

Ao professor Humberto Breves Coda pela orientação, pelo basto conhecimento transmitido, pela paciência e as valiosas conversas nos momentos necessários.

Aos grandes amigos do SET que fizeram parte importante deste processo e que ficam para o resto da vida. Especialmente à minha família São Carlos: Juan Camilo, Sergio Andrés e Silvia Margarita.

A todos os professores do SET que aportaram, com grande disposição e amabilidade, à minha formação.

Ao departamento de Engenharia de Estruturas da Escola de Engenharia de São Carlos da Universidade de São Paulo e aos seus funcionários.

À coordenação de Aperfeiçoamento de Pessoal de Nível Superior (CAPES) pela apoio financeiro. 

"Lo difícil se hace inmediatamente, [...] lo imposible lleva un poco más de tiempo".

(Mario Moreno) 



\section{RESUMO}

MORANTES, E.A. Desenvolvimento de modelo de ligação deslizante para a simulação de dispositivos de controle de vibração na análise não linear geométrica de estruturas. 2017. 124 p. Dissertação (Mestrado em Engenharia de Estruturas) Departamento de Engenharia de Estruturas, Escola de Engenharia de São Carlos, Universidade de São Paulo, São Carlos, 2017.

Neste estudo se propõe o desenvolvimento de um modelo numérico para a ligação deslizante entre elementos sólidos bidimensionais, aplicável à simulação de sistemas deslizantes de isolação de base para estruturas. A formulação implementada é baseada no Método dos Elementos Finitos Posicional (MEFP) para análise dinâmica não linear geométrica de estruturas escrita na forma Lagrangeana total. Elementos triangulares planos e isoparamétricos de aproximação cúbica com matriz de massa completa são utilizados principalmente na elaboração da parte sólida dos dispositivos de ligação entre estruturas reticuladas e a base móvel. Esses elementos também poderm ser utilizados na modelagem da estrutura em si, porém, para esse fim, elementos finitos isoparamétricos de barra geral com massa distribuída por unidade de comprimento foram implementados. As equações de movimento são integradas no tempo aplicando o método de Newmark e o problema de deslizamento é resolvido com o algoritmo baseado na técnica dos multiplicadores de Lagrange, onde a restrição das posições de um nó escravo é feita em relação a uma sequência de superfícies mestres. Elementos de barra geral foram usados para simular as superfícies mestres de contato, o que aumenta as possibilidades de aplicações, incluindo mecanismos compostos apenas por barras gerais. Analisam-se exemplos disponíveis na literatura para a validação da formulação proposta e propõem-se aplicações diversas na engenharia das estruturas.

Palavras-chave: Método dos elementos finitos posicional. Análise não linear geométrica. Ligações deslizantes. Dinâmica das estruturas e mecanismos. Controle de vibração. 



\section{ABSTRACT}

MORANTES, E.A. Development of sliding joint model for simulation of vibration control devices in geometric nonlinear analysis of structures. 2017. 124 p. Dissertation (Master in Structural Engineering) - Departament of Structural Engineering, Escola de Engenharia de São Carlos, Universidade de São Paulo, São Carlos, 2017.

This study proposes the development of a numerical model for the sliding joint between two-dimensional solid elements, applicable to the simulation of sliding base isolation systems. The implemented formulation is based on the Positional Finite Element Method (PFEM) for geometrical nonlinear dynamic analysis of structures written in the total Lagrangian form. Plane and isoparametric triangular cubic approximation elements with full mass matrix are mainly used in the elaboration of the solid part of the devices of joints between reticulated structures and mobile base. These elements can also be used in the modeling of the structure itself, however, for that purpose, isoparametric elements of general bar with mass distributed per unit of length were implemented. The motion equations are integrated in time by applying the Newmark method and the sliding problem is solved with the algorithm based on the technique of Lagrange multipliers, where the constraint of the positions of a slave node is made in relation to a sequence of master surfaces. General bar elements were used to simulate the master contact surface, which increases the possibilities of applications, including mechanisms composed only of general bars. Analyze examples available in the literature for the validation of the proposed formulation and proposed diverse applications in the engineering of the structures.

Keywords: Positional finite element method. Geometrical nonlinear analysis. Sliding joints. Dynamics of structures and mechanisms. Vibration control. 



\section{LISTA DE FIGURAS}

FigurA 1 - (A) PENDULO de ATRITO SIMPLES. (B) TMD INSTALADO NO PRÉDIO TAIPEI 101.

FIGURA 3 -DETALHE DO PENDULO DE ATRITO SIMPLES.

FIGURA 4 - (A) CONDIÇÃO DESLOCADA DO FPS (B) REPRESENTAÇÃO DA RIGIDEZ LATERAL DO FPS.

FIGURA 2 -IDENTIFICAÇÃO DOS COMPONENTES DO DISPOSITIVO FPS PARA A MODELAGEM.

Figura 5 - Modelo KelVIN/Voight MOdificado. Tomado de MAdeira e Coda (2016) .44

FIGURA 6 - DisPositivo MASSA/MOLA/AMORTECEDOR. TOMADO DE MADEIRA E CODA (2016). .46

FIGURA 7 - TRELIÇA DE VON MISES. .49

FIGURA 8 - TRAJETÓRIA DE EQUILÍBRIO DO NÓ B DA TRELIÇA DE VON MISES. 50

FIGURA 9 - GEOMETRIA DO EXEMPLO DE VALIDAÇÃO DISPOSITIVO

MASSA/MOLA/AMORTECEDOR. TOMADO DE MADEIRA E CODA (2016). 51

FIGURA 10 - RESPOSTA QUASE-ESTÁTICA DO DISPOSITIVO.

FIGURA 11 - MAPEAMENTO DAS CONFIGURAÇÕES INICIAL E ATUAL E ATUAL E SUA

REPRESENTAÇÃO NO ESPAÇO ADIMENSIONAL.

FIGURA 12 - VIGA ENGASTADA SUBMETIDA A UM CARREGAMENTO TRANSVERSAL VARIÁVEL NO TEMPO.

FIGURA 13 - FORÇA TRANSVERSAL APLICADA NO EXTREMO LIVRE DA VIGA VERSUS TEMPO.

FIgURA 14 - DESLOCAMENTO HORIZONTAL PARA VALORES DE FORÇA APLICADA MÁXIMA DE

$\mathrm{F}=1 \mathrm{E}+05 \mathrm{E} F=5 \mathrm{E}+05$ LIBRAS.

FIGURA 15 - DESLOCAMENTO VERTICAL PARA VALORES DE FORÇA APLICADA MÁXIMA DE

$$
\mathrm{F}=1 \mathrm{E}+05 \mathrm{E} F=5 \mathrm{E}+05 \text { LIBRAS. }
$$

FIGURA 16 - CONFIGURAÇÃO DESLOCADA T $=0.25$ SEGUNDOS. .62

FIGURA 17 - CONFIGURAÇÃO DESLOCADA PARA T=0.35 SEGUNDOS... 63

FIGURA 18 - DESLOCAMENTO VERTICAL DO EXTREMO LIVRE DA VIGA. 64

FIGURA 19. MAPEAMENTO DAS CONFIGURAÇÕES E A SUA RELAÇÃO COM O ESPAÇO 
FIGURA 20 - ESQUEMA PARA ANÁLISE DE FLAMBAGEM DE COLUNA.

FIGURA 21 - (A) CURVA ADIMENSIONAL FORÇA-DESLOCAMENTO TRANSVERSAL; (B) CURVA

ADIMENSIONAL FORÇA-DESLOCAMENTO LONGITUDINAL.

FIGURA 22 - EVOLUÇÃO DO DESLOCAMENTO TRANSVERSAL E CONFIGURAÇÃO DESLOCADA

PARA DIFERENTES PORCENTAGENS DE FORÇA APLICADA. .74

FIGURA 23 - ESQUEMA DO MECANISMO BIELA-MANIVELA. .75

FIGURA 24 - HISTÓRICO DA POSIÇÃO HORIZONTAL DO APOIO DIREITO PARA AS FUNÇÕES DE MOMENTO APLICADAS. .77

FIGURA 25 - EVOLUÇÃO DO DESLOCAMENTO HORIZONTAL E CONFIGURAÇÃO DESLOCADA PARA DIFERENTES INSTANTES DE TEMPO AO APLICAR A FUNÇÃO DE MOMENTO M2(T). 77 FIGURA 26 - DESCRIÇÃO ESQUEMÁTICA DAS LIGAÇÕES DESLIZANTES. (A) LIGAÇÃO PRISMÁTICA. (B) LIGAÇÃO CILÍNDRICA. TOMADO DE SIQUEIRA (2016). .79

FIGURA 27 - ACOPLAMENTO ENTRE ELEMENTOS SÓLIDOS BIDIMENSIONAIS E ELEMENTOS DE PÓRTICO. .86

FIGURA 28 - ESQUEMA DA ESTRUTURA PARA ANÁLISE DE FLAMBAGEM APLICANDO FORÇA DE TRAÇÃO. TOMADO DE SIQUEIRA (2016) .88

FIGURA 29 - EVOLUÇÃO DO ÂNGULO DE INCLINAÇÃO DA BARRA RÍGIDA. .89

FIGURA 30 - EVOLUÇÃO DO DESLOCAMENTO HORIZONTAL DO APOIO DIREITO. .89

FIgURA 31 - ESQUEMA DO MECANISMO DE RETORNO. TOMADO DE SIQUEIRA (2016).........90

FIGURA 32 - VELOCIDADE VERTICAL DO NÓ N. 91

FIGURA 33 - DEFLEXÃO DO BRAÇO NO PONTO A.

FIgURA 34 - TERREMOTO 'SUPERSTITION HILLS, 1987'. COMPONENTE HORIZONTAL E VERTICAL DOS DESLOCAMENTOS. .94

FiguRA 35 - - (A) MOdo 1. (B) Modo 2. (C) Modo 3. 95

FIGURA 36 - ESTRUTURA DEFORMADA (A) CARREGAMENTO ESTÁTICO. (B) ANÁLISE DINÂMICA $\mathrm{T}=7.19[\mathrm{~S}]$. .96

FIGURA 37 - ANÁLISE DINÂMICA (A) T = 12.36 [S] (B) T = 21.99 [S] ............................

FIGURA 38 - DESLOCAMENTO HORIZONTAL. .97

FIGURA 39 - ACELERAÇÃO HORIZONTAL O TOPO DA ESTRUTURA COMO FRACÇÃO DA GRAVIDADE. 98

FIGURA 40 - DESLOCAMENTO HORIZONTAL DO PILAR DO EIXO ESQUERDO. .98

FIgURA 41 - DETALHE DO DISPOSTIVO FPS SELECIONADO. .99

FIGURA 42 - DISCRETIZAÇÃO DO DISPOSITIVO FPS. .99 
FIGURA 43 - DESCRIÇÃO GRÁFICA DO ACOPLAMENTO DOS ELEMENTOS DE PÓRTICO NAS

FACES DESLIZANTES DOS SÓLIDOS BIDIMENSIONAIS.

FIGURA 44 - MALHA DE ELEMENTOS FINITOS PARA ANÁLISE DA ESTRUTURA ISOLADA COM DISPOSITIVOS FPS.

FIGURA 45 - DESLOCAMENTO HORIZONTAL E VERTICAL DURANTE O TERREMOTO

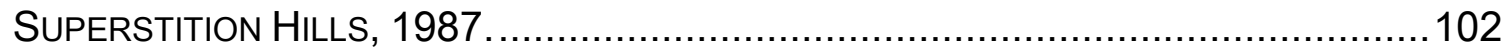

FIGURA 46 - DESLOCAMENTO HORIZONTAL. ..................................................... 103

FIGURA 47 - ACELERAÇÃO HORIZONTAL DO TOPO DA ESTRUTURA. ............................. 104

FIGURA 48 - DESLOCAMENTO HORIZONTAL DO PILAR DO EIXO ESQUERDO.....................104

FIGURA 49 - FORÇA DE CONTATO ENTRE O DESLIZADOR ARTICULADO E A SUPERFÍCIE

CÔNCAVA NO FPS ESQUERDO. (A) SOMENTE CARREGAMENTO ESTÁTICO (B) INSTANTE DO MÁXIMO DESLOCAMENTO HORIZONTAL 15.625 SEGUNDOS (C) INSTANTE DO MÍNIMO DESLOCAMENTO HORIZONTAL 17.065 SEGUNDOS (D) INSTANTE DE DESLOCAMENTO HORIZONTAL NULO 16.305 SEGUNDOS. 105

FIGURA 50 - DEFORMADA APÓS A APLICAÇÃO DO CARREGAMENTO ESTÁTICO ( T = 0.0S). (A)

ISOLADOR ESQUERDO (B). ISOLADOR DIREITO. 106

FIGURA 51 - DEFORMADA INSTANTE T = 15.625 S (A) ISOLADOR ESQUERDO (B) ISOLADOR DIREITO. 107

FIGURA 52 - DEFORMADA INSTANTE T = 17.065 S (A) ISOLADOR ESQUERDO (B) ISOLADOR DIREITO. 108

FIGURA 53 - DEFORMADA INSTANTE T = 16.305 S (A) ISOLADOR ESQUERDO (B) ISOLADOR DIREITO. 109

FIGURA 54 - DESLOCAMENTO HORIZONTAL RELATIVO MEDIDO ENTRE O CENTRO DO CONTORNO DA SUPERFÍCIE CÔNCAVA E O CENTRO DO CONTORNO DO DESLIZADOR ARTICULADO. 110

FIGURA 55 - DESLOCAMENTO HORIZONTAL.

FIGURA 56 - FORÇA DE CONTATO ENTRE O DESLIZADOR ARTICULADO E A SUPERFÍCIE CÔNCAVA NO FPS ESQUERDO. (A) SOMENTE CARREGAMENTO ESTÁTICO (B) INSTANTE DO MÁXIMO DESLOCAMENTO HORIZONTAL 15.625 SEGUNDOS (C) INSTANTE DO MÍNIMO DESLOCAMENTO HORIZONTAL 17.065 SEGUNDOS (D) INSTANTE DE DESLOCAMENTO HORIZONTAL NULO 16.305 SEGUNDOS.

FIGURA 57 - DETALHE DO MODELO DE ELEMENTOS FINITOS DA ESTRUTURA COM O SISTEMA TMD. 


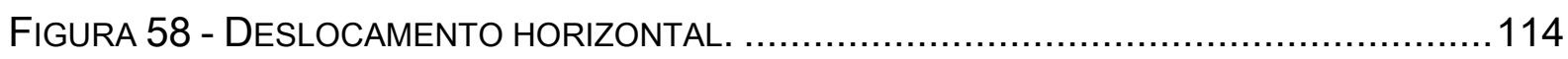

FIGURA 59 - DESLOCAMENTO HORIZONTAL DO PILAR DO EIXO ESQUERDO....................115

FIGURA 60 - ACELERAÇÃO HORIZONTAL DO TOPO DA ESTRUTURA. ...............................115

FIGURA 61 - ACELERAÇÃO HORIZONTAL DO TMD. ........................................... 116

FIGURA 62 - DETALHE DO MODELO DE ELEMENTOS FINITOS. .................................117

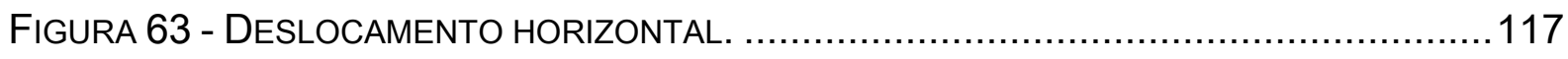




\section{LISTA DE SÍMBOLOS}

O significado dos símbolos empregados no desenvolvimento da formulação implementada encontra-se descrito no texto. 



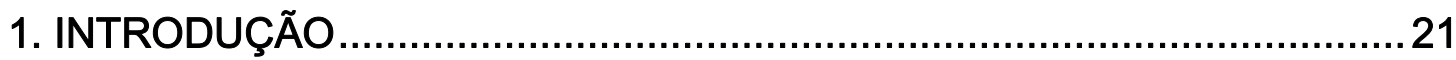

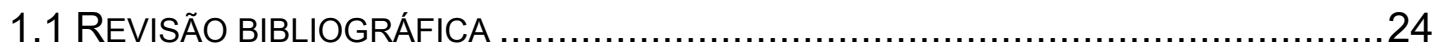

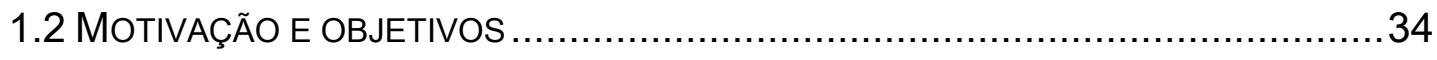

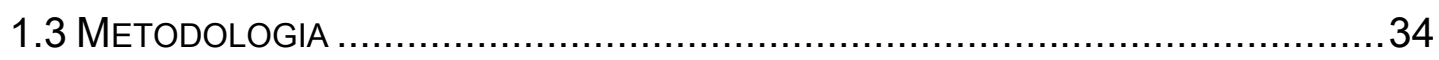

2. FORMULAÇÃO POSICIONAL DO MÉTODO DOS ELEMENTOS FINITOS PARA ANÁLISE NÃO LINEAR GEOMÉTRICA DE ESTRUTURAS ...............37

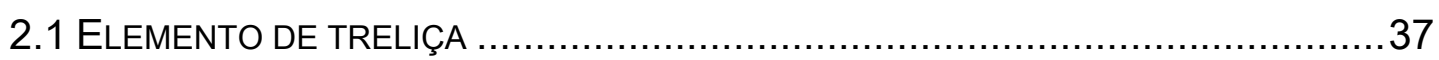

2.1.1 Calculo da força inercial e da força de amortecimento viscoso.......38

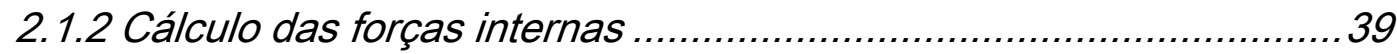

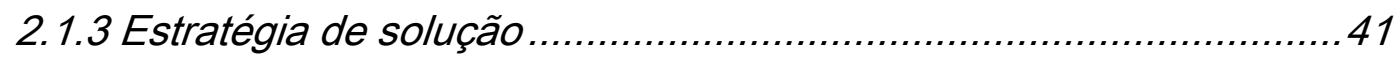

2.1.4 Modelo de amortecimento Kelvin/Voight modificado .......................44

2.1.5 Dispositivo massa/mola/amortecedor .........................................46

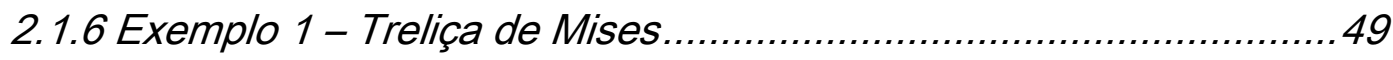

2.1.7 Exemplo 2 - Validação do dispositivo massa/mola/amortecedor. ...50

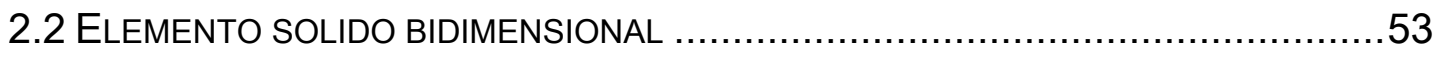

2.2.1 Forças internas do elemento tipo chapa.....................................54

2.2.2 Matriz hessiana do elemento tipo chapa ....................................57

2.2.3 Matriz de massa do elemento tipo chapa .....................................59

2.2.4 Exemplo 1 - Viga engastada submetida a um carregamento

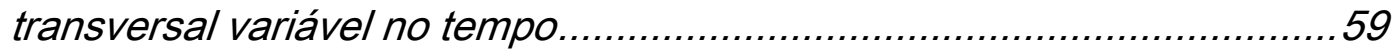

2.2.5 Exemplo 2 - Viga engastada com amortecimento submetida a um carregamento transversal variável no tempo.........................................63

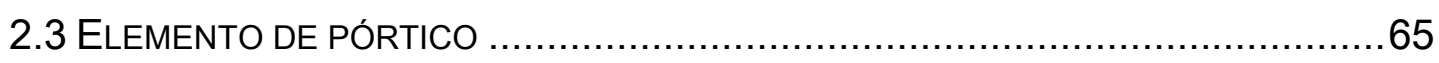

2.3.1 Forças internas do elemento tipo pórtico.......................................68

2.3.2 Matriz hessiana do elemento tipo pórtico .................................... 70

2.3.3 Matriz de massa do elemento tipo pórtico ..................................... 71

2.3.4 Exemplo 1 - Flambagem elástica de coluna ............................... 72

2.3.5 Exemplo 2 - Mecanismo biela-manivela .................................... 75

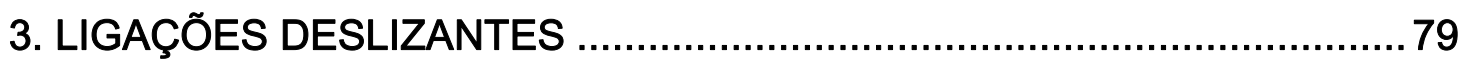


3.1 RESTRIÇÃO CINEMÁTICA VIA MULTIPLICADORES DE LAGRANGE ....................80

3.2 CÁLCULO DA VARIÁVEL CURVILÍNEA E ADIMENSIONAL .............................8 84

3.3 CONSIDERAÇÕES ADICIONAIS PARA A MODELAGEM DE DESLIZAMENTO ENTRE

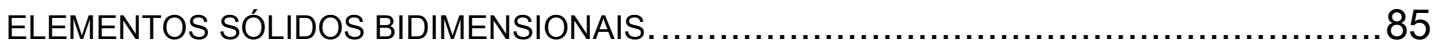

3.4 EXEMPLO 1 - FLAMBAGEM DE UMA ESTRUTURA APLICANDO FORÇA DE TRAÇÃO .87

3.5 EXEMPLO 2 - MECANISMO DE RETORNO RÁPIDO. .90

\section{APLICAÇÕES: MODELAGEM DE SISTEMAS DE CONTROLE DE} VIBRAÇÃO

4.1 ESTRUTURA ENGASTADA NA BASE SUBMETIDA A TERREMOTO. 94

4.2 ESTRUTURA ISOLADA NA BASE COM DISPOSITIVOS FPS. .99

4.2.1 Caso a. Estrutura isolada na base com dispositivos FPS submetida às componentes horizontal e vertical de um terremoto real. 102

4.2.2 Caso b. Estrutura isolada na base com dispositivos FPS submetida à componente horizontal de um terremoto real. 111

4.3 ESTRUTURA COM SISTEMA TMD ................................................. 113

4.4 ESTRUTURA COM SISTEMA TMD E ISOLADA COM DISPOSITIVOS FPS. 116

5. CONCLUSÕES 119

5.1 TRABALHOS FUTUROS. 121 


\section{INTRODUÇÃO}

O controle estrutural de vibrações induzidas por sismos e vento pode ser exercido modificando as propriedades dinâmicas da estrutura: rigidez, massa e amortecimento. Com esse objetivo, têm surgido vários dispositivos baseados nos conceitos de isolamento e absorção.

Nas estruturas civis, mudar a massa da estrutura pode ser uma alternativa inviável, já que na maioria das vezes questionaria o uso para o qual a estrutura está sendo projetada. Portanto, a abordagem clássica na pratica é modificar a rigidez aumentando o tamanho das seções transversais ou adicionando elementos estruturais. Continuando com o mesmo enfoque e aproveitando os avanços da ciência dos materiais, surgiram outras técnicas de reforço de estruturas que empregam materiais de alta resistência ou ductilidade, por exemplo, os tecidos de fibra de carbono, aramida ou vidro.

A outra abordagem para o controle de vibrações em estruturas civis encontrase fortemente vinculada às áreas do conhecimento da engenharia mecânica e aeronáutica. Dado que a introdução dos conceitos de isolamento, amortecimento e absorção deve-se ao motor de combustão interna, desenvolvido no início do século $X X$, e às estruturas de aeronaves usadas durante a segunda guerra mundial. Seguidamente, na década de 1960, a engenharia de estruturas adaptou esses conceitos para aplica-los às estruturas civis localizadas em regiões de ameaça sísmica ou com presença de fortes ventos (HOUSNER et al., 1997).

A formalização do conceito de controle estrutural como uma alternativa segura no projeto de estruturas civis atribui-se ao trabalho de Yao (1972), dando início a inumeráveis pesquisas focadas em otimizar o comportamento dos dispositivos ou nos métodos de análise.

Os avanços nessa direção têm sido tão significativos que atualmente existe um grande número de sistemas de controle. Alguns autores os classificam em três grupos: sistemas de controle ativo, passivo, e hibrido, sendo esse último uma combinação dos dois anteriores. Os sistemas ativos caracterizam-se por usar uma fonte externa de potência para realizar a ação de controle, encontram-se nesse 
grupo os tendões ativos, tirantes ativos e sistemas de amortecedores de massa ativos. Em contrapartida, os sistemas passivos dispensam de energia externa, tais como: isoladores de base, amortecedores de massa sintonizada, amortecedores metálicos de cedência, amortecedores visco-elásticos, amortecedores de fluido viscoso e de atrito (HOUSNER et al., 1997).

Esse trabalho interessa-se especificamente em dois dispositivos de controle de vibração. O primeiro deles, o Friction Pendulum System (FPS) que consiste em apoiar a estrutura em uma superfície côncava deslizante com um coeficiente de atrito baixo que se comporte como uma ligação flexível perante forças horizontais (ZAYAS et al., 1990). Assim sendo, essa técnica aplica o conceito de isolamento para controlar a amplitude de vibração.

O segundo sistema de controle a simular é o Tuned Mass Damper (TMD) que consiste em ancorar um sistema massa/mola/amortecedor à estrutura primaria e sintonizar sua frequência natural à frequência de excitação. Desta forma, o sistema auxiliar absorve grande parte da energia demandada (HARTOG, 1985).

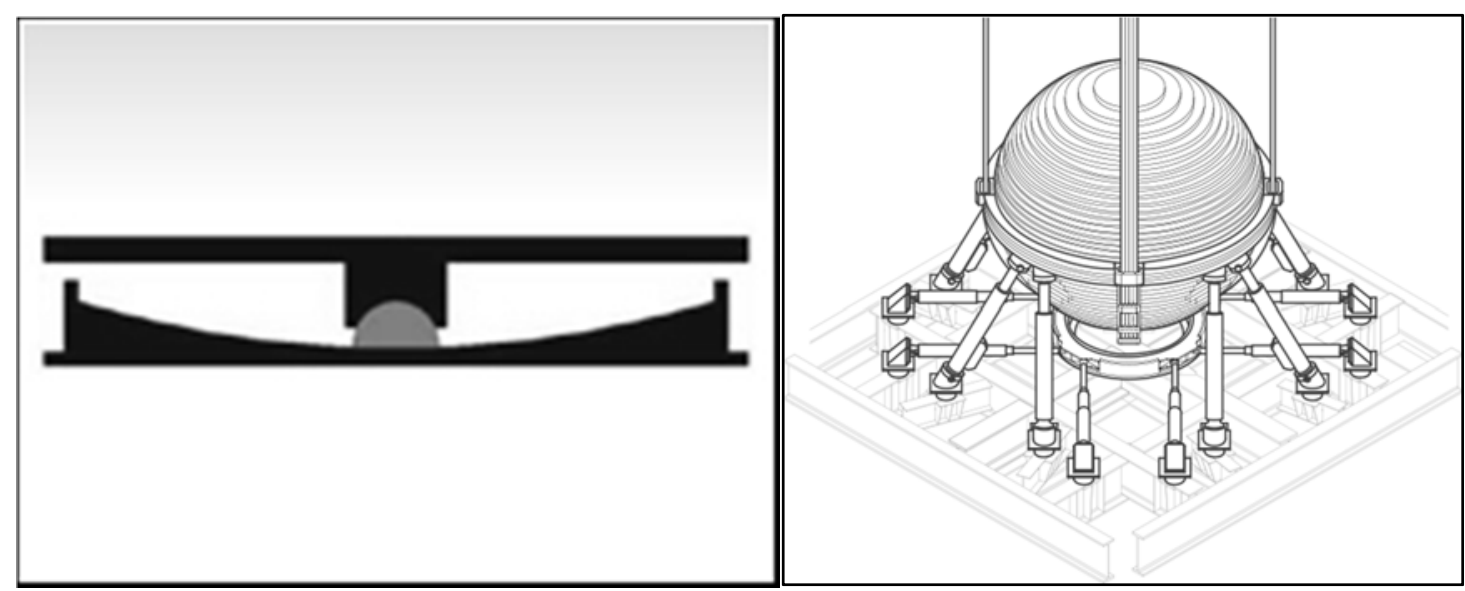

Figura 1 - (a) Pendulo de atrito simples. ${ }^{1}$ (b) TMD instalado no prédio Taipei $101 .{ }^{2}$

1 Disponível em: http://www.earthquakeprotection.com/triple_vs_single_pendulum.html. Consultado em Set 2016.

2 Disponível em: http://www.taipei-101.com.tw/images/ob-damper-system-0.png. Consultado em Set 2016. 
No que diz respeito à simulação numérica, nas últimas décadas múltiplos trabalhos aplicando o método dos elementos finitos demostram a sua versatilidade na solução de uma ampla faixa de problemas de engenharia. Entre eles, evidentemente, encontram-se os problemas de dinâmica das estruturas.

A simulação de estruturas civis convencionais parte da hipótese linear em deslocamentos, uma aproximação razoável para esse tipo de casos. No entanto, a simulação numérica de estruturas com dispositivos de controle de vibração, como os descritos nos parágrafos anteriores, exige que essa hipótese não seja diretamente aplicada, já que em geral, os dispositivos sofrem grandes deslocamentos por meio dos quais se consegue a dissipação de energia, o isolamento da estrutura ou a absorção da energia, dependendo do caso estudado.

Por esse motivo, alguns autores propuseram estratégias alternativas de modelagem para cada dispositivo, que buscam manter a praticidade do método escrito na sua forma geometricamente linear. No caso do FPS, destaca-se o trabalho de Nagarajaiah et al. (1991), que sugere analisar tanto a estrutura quanto o dispositivo com as hipóteses validas para pequenos deslocamentos e introduzir as forças de interação por meio de elementos com relação constitutiva não linear localizados no nível dos dispositivos de isolamento.

Para modelar o TMD, a metodologia mais empregada também parte das hipóteses de pequenos deslocamentos e transforma o sistema de múltiplos graus de liberdade da estrutura em um sistema de um grau de liberdade equivalente ao qual vincula-se o sistema auxiliar. Desse modo, analisa-se a resposta de um sistema de dois graus de liberdade (SOONG; DARGUSH, 1997).

Fazer uma modelagem mais completa e precisa implica a consideração da não linearidade geométrica na formulação do método dos elementos finitos. Além disso, em alguns casos deve ser tratada a interação entre os corpos, estrutura e dispositivos de controle, para o qual tem que apelar-se às técnicas de solução da mecânica de multicorpos flexíveis.

No que diz respeito à consideração da não linearidade geométrica em estruturas, a formulação co-rotacional do método dos elementos finitos tem sido mais empregada na literatura, a qual, apesar de ter vários trabalhos que validam os resultados obtidos, mostra-se como uma alternativa complexa na sua 
implementação computacional. Nesse trabalho emprega-se a formulação posicional do método dos elementos finitos proposta por Coda (2003) que, por ser Lagrangiana total, apresenta operacionalidade mais simples que suas análogas.

\subsection{Revisão bibliográfica}

Os sistemas de controle passivo possuem grandes vantagens como: ser usualmente econômicos, apresentar inerente estabilidade e dispensar uma fonte externa de energia, já que ela nem sempre é garantida, especialmente durante eventos extremos como terremotos, vendavais ou furações (HOUSNER et al, 1997)

A estratégia da maioria dos sistemas de controle de vibração consiste em concentrar o comportamento não linear nos dispositivos para conseguir a maior isolação, dissipação ou absorção de energia, dessa forma, a demanda de força, deslocamentos e/ou deformações na estrutura primaria se reduz.

Esse trabalho interessa-se na modelagem de dois dispositivos usados extensivamente como sistemas de controle estrutural passivo. O primeiro deles é um sistema de isolamento de base para pontes e estruturas de baixa e mediana altura chamado de pêndulo de atrito simples, desenvolvido em 1985 pelo engenheiro Ph.D Victor Zayas para prevenir colapso e danos excessivos em estruturas submetidas a eventos sísmicos (ZAYAS; LOW; MAHIN, 1987). Consiste em um deslizador articulado revestido com PTFE (Politetrafluoretileno) assentado sobre uma superfície esférica côncava recoberta por aço inoxidável polido (na figura 3 , refere-se ao deslizador articulado como 'Moving Disc'). 


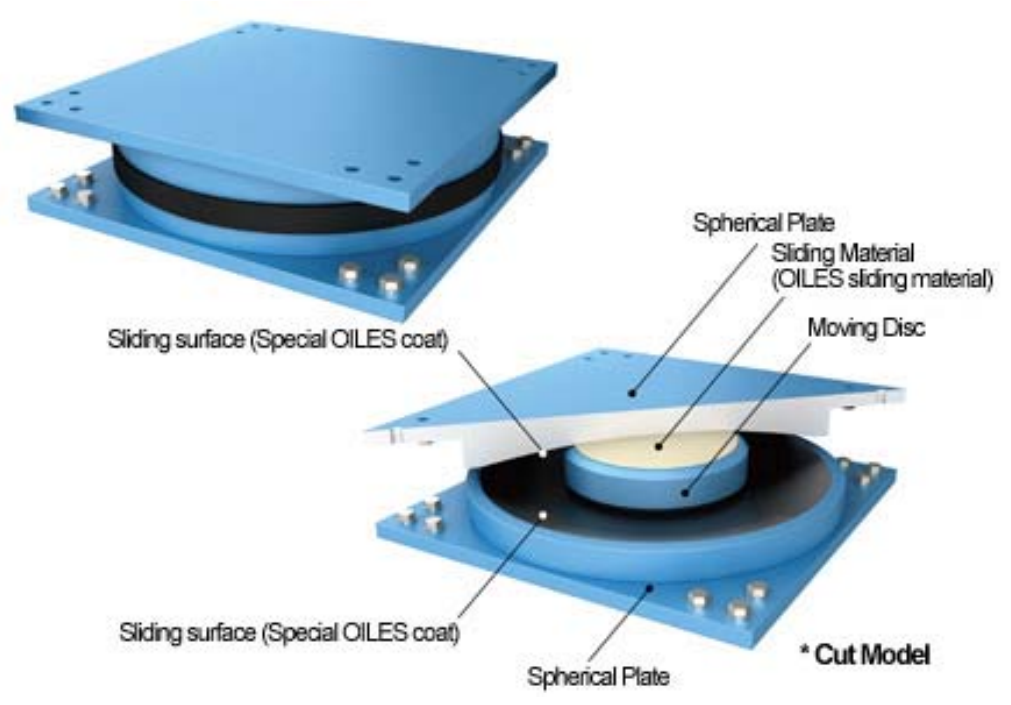

Figura 2 -Detalhe do pendulo de atrito simples 3.

O nível de instalação dos dispositivos, usualmente na base, chama-se de nível de isolamento. Nesse nível os dispositivos fazem a estrutura altamente flexível perante forças horizontais, dessa forma, desacopla-se a estrutura das altas frequências de vibração do solo, que se traduz em uma redução nas acelerações relativas da estrutura primaria. Além disso, o modo de vibração predominante da estrutura passa a ser um movimento de corpo rígido cujo período de vibração definese com a geometria do dispositivo (FPS).

$\mathrm{Na}$ condição deslocada (ver figura 3), a rigidez lateral do dispositivo é dada pela relação: peso suportado/raio de curvatura da superfície côncava. Percebe-se que a rigidez é diretamente proporcional ao peso suportado, logo, o centro de rigidez coincide com o centro de massa e, portanto, os efeitos torcionais são mínimos, inclusive em estruturas fortemente assimétricas. No trabalho numérico-experimental de Zayas et al. (1990) verificou-se essa propriedade do sistema FPS.

3 Disponível em: http://www.oiles.co.jp/en/menshin/building/menshin/products/fps/img/indexfigure-01.jpg. Consultado em Fev 2017. 


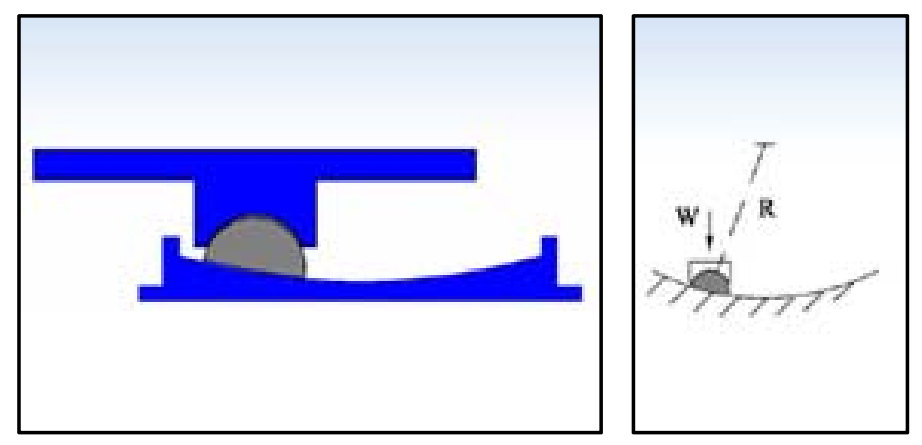

Figura 3 - (a) Condição deslocada do FPS (b) Representação da rigidez lateral do FPS4.

No mesmo trabalho de Zayas et al. (1990) comenta-se outra propriedade do dispositivo FPS, especificamente relacionada com a geometria do deslizador articulado. A configuração semiesférica do deslizador resulta em uma distribuição uniforme de pressões na superfície de contato para qualquer tipo de solicitação vertical e horizontal. Assim evita-se o efeito de cinzelamento das superfícies e se reduzem as altas frequências de movimento do efeito 'Stick-slip', presente nos dispositivos deslizantes similares.

Mais tarde no trabalho experimental de Mokha et al. (1991) testou-se uma estrutura de seis pavimentos isolada com sistemas de pêndulos de atrito simples. Ressaltam-se duas conclusões, a primeira delas em relação ao comportamento elástico da estrutura afirmando: "O sistema é efetivo na proteção do sistema estrutural sob condições de força sísmica extrema com conteúdo de frequência significativamente diferente. Em todos os testes o modelo se manteve elástico". E a seguinte conclusão foi apresentada a respeito da natureza do contato apresentado: "Não ocorreu levantamento no isolador apesar da grande relação entre a altura e a separação dos isoladores".

Destacam-se também os trabalhos experimentais de Constantinou et al. (1990), Bondonet; Filiatrault (1997) e Constantinou et al. (1999) para calibrar um modelo matemático que represente o comportamento friccional do teflon nos dispositivos deslizantes, isto é, as forças de interação entre o dispositivo e a estrutura.

4 Disponível em: http://www.earthquakeprotection.com/product2.html. Consultado em Fev 2017. 
Em relação à simulação numérica de um sistema estrutural com dispositivos de isolamento de base o trabalho de Nagarajaiah et al. (1991) apresenta um algoritmo de solução onde se propõe representar a estrutura primaria como sendo elástica linear e a não linearidade da relação força-deslocamento horizontal, presente no nível de isolamento devido aos FPS instalados, é introduzida por meio de um vetor de pseudo-forças não lineares corrigido iterativamente. Aplica-se o método de Newmark como integrador temporal e o método de Runge-Kutta para solucionar as equações diferenciais que governam o comportamento não linear dos elementos de isolação. Essa estratégia de solução tem sido implementada em alguns softwares comerciais para análise de estruturas que empregam o método dos elementos finitos.

Nota-se que essa abordagem se baseia em leis constitutivas para pequenos deslocamentos dos dispositivos de isolamento. Apesar das hipóteses de pequenos deslocamentos terem se mostrado precisas em estimar a resposta global da estrutura, o trabalho de Almazán et al. (1998) mostra que um dos aspectos mais importantes na simulação numérica de estruturas com isoladores FPS é a avaliação precisa da força normal gerada pela restrição cinemática imposta pela superfície de deslizamento e para tanto, o modelo numérico deve levar em consideração a componente vertical do solo e o acoplamento real do movimento vertical e horizontal da estrutura.

Além disso, nos terremotos de Northridge (1994), Kobe (1995), Turkey (1999) e Taiwan (1999) observaram-se grandes deslocamentos que exigem a avaliação dos efeitos não lineares geométricos (usualmente chamados de $\mathrm{P}-\Delta$ nos trabalhos da prática) associados no dimensionamento desse tipo de estruturas. Em vista disso, no trabalho de Almazán e De la Llera (2002) apresenta-se um modelo analítico, especificamente para dispositivos FPS, que considera os efeitos mencionados anteriormente, incluindo as forças de atrito, e emprega a formulação aumentada da dinâmica de multicorpos para a imposição das restrições cinemáticas.

No trabalho de Clarke et al. (2005), utilizou-se um elemento conhecido como superfície rígida analítica, disponível no software ABAQUS, que cria um contato deslizante mestre/escravo entre nós coincidentes. A superfície de deslizamento côncava do pendulo foi representada por uma superfície rígida, enquanto o 
deslizador convexo definiu-se como o elemento escravo. A ligação articulada entre o deslizador e o sólido superior, ou melhor, o acoplamento dos graus de liberdade translacionais foi definido implicitamente.

O segundo dispositivo de interesse específico desse trabalho é o amortecedor de massa sintonizado (Tuned Mass Damper - TMD). Este dispositivo é um absorvedor clássico de vibrações e consiste em uma massa auxiliar (aproximadamente $1 \%$ da massa total da estrutura primaria) instalada no topo da estrutura e conectada por meio de amortecedores e molas passivas. Segundo Housner et al. (1997), atribui-se a introdução dessa estratégia na engenharia civil a Hartog (1985).

Os desenvolvimentos iniciais estiveram limitados ao uso de absorvedores dinâmicos na indústria de engenharia mecânica, onde se tem uma frequência de operação em ressonância que coincide com a frequência fundamental da máquina. Porém, estruturas civis estão sujeitas a carregamentos ambientais, tais como vento e terremotos, que se caracterizam por compreender uma larga banda de frequências. Por conseguinte, muita pesquisa foca-se no estudo da eficiência do TMD operando nessas vibrações ambientais. Para o cálculo dos parâmetros ótimos do TMD destacam-se as seguintes estratégias: aceleração mínima da estrutura principal, velocidade mínima da estrutura principal, deslocamento mínimo da estrutura principal, força mínima dos elementos da estrutura principal (WARBURTON, 1982), máxima rigidez dinâmica da estrutura principal e máximo amortecimento efetivo do sistema estrutura/TMD combinado, entre outros.

Nota-se ainda que a estratégia de modelagem principalmente empregada na literatura é representar a estrutura como um oscilador elástico de um grau de liberdade com um sistema auxiliar ligado. Soong \& Dargush (1997) apresentam algumas estratégias para o cálculo dos parâmetros ótimos do TMD e o processo de transformação do sistema de múltiplos graus de liberdade em um sistema de um grau de liberdade equivalente ao qual se acopla o TMD. Além disso, se discute o problema quando o primeiro modo de vibração não exibe uma participação alta na resposta, dado que a influência dos modos altos de vibração começam a ser preponderantes. 
Atualmente, a prática da engenharia estrutural encontra-se fortemente ligada ao desenvolvimento computacional devido à utilidade dos métodos numéricos na solução de uma ampla faixa de problemas. Destacando-se nas últimas décadas o método dos elementos finitos (MEF). Segundo Cook et al. (2002), o MEF originou-se com o trabalho de Courant (1943) que determinou a rigidez a torção de um eixo oco dividindo a seção transversal em elementos triangulares dentro dos quais empregou funções lineares para interpolar o campo de tensão. Depois disso, grandes avanços na interpretação e nas possíveis aplicações da estratégia surgiram na década de 1950, na qual destaca-se o trabalho de Turner et al. (1956). Finalmente. Em 1960 o nome "elementos finitos" foi empregado pela primeira vez em CLOUGH (1960) que criou, em Berkeley, o primeiro grupo de pesquisa formal nesse assunto para impulsionar a ideia em aplicações na engenharia civil. Em 1963, o MEF adquiriu maior aceitação no meio acadêmico dado que foi reconhecido como uma variante do método de Rayleigh-Ritz, por conseguinte encerra uma base matemática que 0 sustenta.

Desde então começou-se a busca de algoritmos mais eficientes que possibilitassem reduzir o tempo de cálculo e a memória requerida dos computadores. Surgindo assim formulações baseadas no método dos deslocamentos, em modelos de equilíbrios e métodos híbridos ou mistos, sendo o método dos deslocamentos o mais aplicado devido a sua versatilidade e relativa simplicidade (ZIENKIEWICZ; TAYLOR, 1988).

Assim, o MEF tornou-se uma ferramenta extremamente útil para resolver equações diferenciais associadas a problemas físicos com geometrias complexas. No entanto, a resolução de um problema de análise de estruturas precisa de outras definições importantes, tais como o grau flexibilidade da estrutura e o tipo de lei constitutiva do material, dado que esses dois aspectos podem introduzir não linearidades no sistema de equações gerado pela técnica dos elementos finitos. Finalmente, a natureza das solicitações aplicadas define se a análise é estática, quase estática ou dinâmica.

Esse trabalho pode ser enquadrado em um conjunto de formulações associadas à análise estática e dinâmica não linear geométrica de estruturas flexíveis articuladas ou não via método dos elementos finitos. As potencialidades da 
formulação a ser desenvolvida e aplicada possibilitam, inclusive, a análise de multicorpos flexíveis. É interessante observar que as estruturas analisadas apresentam pequenas deformações, apesar de desenvolver grandes deslocamentos, o que permite se adotar lei constitutiva linear baseada na deformação de Green sem alterar o comportamento elástico linear de material constituinte.

Problemas da análise não linear geométrica de estruturas tem sido abordados analítica e numericamente. Devido à complexidade da análise, as soluções analíticas disponíveis na literatura são poucas, quando comparadas com as abordagens numéricas, e restringem-se a estruturas com geometria simples. Destacam-se os trabalhos de Bisshopp e Drucker (1945), Kerr (1964) e Mattiasson (1981) entre outros. Nesse trabalho, as soluções analíticas são utilizadas na validação dos códigos computacionais desenvolvidos.

As soluções numéricas para problemas geometricamente não lineares partem de formulações matemáticas que podem ser classificadas como Lagrangeanas ou Eulereanas, segundo o referencial adotado para a descrição das variáveis envolvidas. Na análise de estruturas, uma descrição Lagrangeana utiliza a configuração inicial como sistema de referência para medir as deformações nos elementos, enquanto uma descrição Eulereana emprega a configuração atual do corpo. Por sua vez, as formulações Lagrangeanas podem ser subdivididas, dependendo da atualização do sistema de referência, como: formulação Lagrangeana atualizada, parcialmente atualizada ou fixa. Os enfoques Lagrangeanos que atualizam o seu referencial diferenciam-se pela forma em que ele é atualizado, sendo chamados de parcialmente atualizados quando a atualização ocorre ao início de cada passo de tempo (M. B. WONG; F. TIN-LOIS, 1990).

A formulação co-rotacional é outra técnica bastante empregada na análise não linear geométrica de estruturas e caracteriza-se por empregar formulações lineares em relação a um sistema de referência responsável pela introdução da não linearidade através da sua própria rotação (CRISFIELD, 1991). A dificuldade dessa formulação deve-se à natureza não vetorial das variáveis da rotação (CRISFIELD, 1990). A formulação co-rotacional pode ser classificada como Lagrangeana atualizada, porém, como já mencionado, os deslocamentos são considerados 
pequenos a partir da configuração atualizadas pelas torações de corpo rígido, veja por exemplo Jiang, Chernuka e Pegg (1994).

No departamento de engenharia de estruturas da Escola de Engenharia de São Carlos (SET) foi proposta uma formulação Lagrangeana total do método dos elementos finitos que utiliza as posições (MEFP) como incógnitas principais, não os deslocamentos, com a vantagem de ser uma formulação com operacionalidade mais simples que as apresentadas por suas análogas (CODA; GRECO, 2004), (MACIEL; CODA, 2005).

Vários trabalhos têm sido desenvolvidos para demostrar a eficiência do método dos elementos finitos posicional para análise não linear geométrica de estruturas, entre eles, podem ser citados os seguintes: Análise não linear geométrica com impacto de domínios elásticos bidimensionais (MARQUES, 2006); Análise não linear geométrica de problemas modelados por pórticos planos e sólidos tridimensionais (MACIEL, 2008); Sobre modelos constitutivos não lineares para materiais com gradação funcional exibindo grandes deformações: implementação numérica em formulação não linear geométrica (PASCON, 2012); Análise não linear geométrica de estruturas com interação fluido-casca (SANCHES, 2011); Análise dinâmica não linear geométrica de estruturas e mecanismos reticulados planos com ligações deslizantes (SIQUEIRA, 2016), entre outros.

Tendo em mente que o presente trabalho é destinado à modelagem 'exata' de sistemas que incluem entidades estruturais complexas (i.e. dispositivos FPS e TMD) que não podem ser representadas diretamente com elementos finitos convencionais (i.e. pórticos, vigas, chapas, treliças, etc.), introduzem-se conceitos de ligações deslizantes, usualmente aplicados na área do conhecimento da dinâmica de multicorpos. Nos seguintes parágrafos comentam-se as estratégias numéricas que permitem tratar problemas que envolvem a interação entre dois ou mais corpos.

As restrições cinemáticas associadas a uma determinada ligação/interação entre corpos representam-se, matematicamente, por meio de equações algébricas ou diferenciais chamadas de equações de compatibilidade. Dependendo do tipo de ligação, algumas delas podem ser expressas explicitamente, de forma que basta empregar um algoritmo de identificação dos graus de liberdade envolvidos para dar solução à cinemática do problema, como pode ser visto no caso das rótulas. O outro 
grupo de ligações caracteriza-se por empregar equações implícitas na sua formulação, desta forma, precisam de técnicas numéricas para sua solução.

As restrições cinemáticas por sua vez classificam-se em holonômicas ou não holonômicas. As primeiras são formuladas como funções implícitas das coordenadas generalizadas e, eventualmente, do tempo. Enquanto, as restrições não holonômicas são escritas na forma de equações diferenciais ou envolvem desigualdades. Fisicamente, a diferença entre esses tipos de condições é que as condições holonômicas ao expressar restrições no número de graus de liberdade, representam um conjunto de configurações possíveis do sistema, enquanto as condições não holonômicas constituem restrições no comportamento de um sistema para ir de uma configuração a outra (GÉRADIN; CARDONA, 2001).

Existem diferentes técnicas numéricas para introduzir as condições de compatibilidade, na literatura encontram-se os seguintes métodos: eliminação das restrições, multiplicadores de Lagrange, funções de penalização, lagrangeano aumentado e lagrangeano perturbado.

Na mecânica computacional, a técnica dos multiplicadores de Lagrange tem sido amplamente empregada para condicionar problemas mecânicos, graças a sua praticidade na implementação computacional quando comparada aos métodos de eliminação de coordenadas dependentes por substituição das equações de compatibilidade. A técnica trabalha com um sistema de equações expandido já que as restrições cinemáticas são adicionadas ao sistema de equações que descreve a mecânica dos corpos, sendo os multiplicadores de Lagrange os termos independentes adicionados ao sistema global. Desta forma, resolve-se simultaneamente as coordenadas generalizadas segundo as quais foi escrito 0 problema e os multiplicadores de Lagrange que, fisicamente, representam a força interna necessária para garantir a condição cinemática imposta.

Assim sendo, infere-se que um problema dinâmico com restrições cinemáticas impostas via multiplicadores de Lagrange envolve dois subsistemas acoplados, um deles descrevendo o comportamento dinâmico por meio das equações diferencias de equilíbrio e o outro subsistema composto pelas equações algébricas de restrição cinemática. 
Com o objetivo de melhorar o condicionamento numérico da técnica dos multiplicadores de Lagrange surgiram o método de Lagrange aumentado (CARDONA et al, 1991) e o método de Lagrange perturbado. O primeiro deles adiciona uma função de penalização com amplitude moderada e um escalamento da restrição cinemática que garante a mesma ordem de magnitude nas matrizes do sistema. De forma análoga, o método lagrangeano perturbado introduz um termo chamado de regularização que gera uma contribuição positiva definida na diagonal da matriz hessiana dos multiplicadores de Lagrange (GÉRADIN; CARDONA, 2001).

Em relação à integração temporal de sistemas de equações diferenciais e algébricas, vários trabalhos têm demonstrado que a maioria de algoritmos disponíveis apresentam instabilidade numérica ou altas frequências de oscilação indesejáveis de origem exclusivamente numérica. Isto se deve à rigidez das equações da dinâmica das estruturas e ao fato de que os multiplicadores de Lagrange não estão associados a uma massa. Uma fonte de imprecisão nas integrações temporais de estratégias Lagrangeanas atualizadas é o fato da matriz de massa não ser constante, pois depende da rotação da referência atualizada. Este problema não existe na formulação posicional. No ambiente das formulações Lagrangeanas atualizadas, surgiram algoritmos alternativos que permitem contornar esses problemas. Destacam-se aqueles que introduzem amortecimento numérico, e outros formulados para conservar ou decair a energia do sistema. Para uma abordagem mais detalhada dos diversos integradores temporais usados em formulações Lagrangeanas atualizadas recomendam-se as referências (GÉRADIN; CARDONA, 2001) (SIMO; TARNOW, 1992) (ROMERO; ARMERO, 2002) (ROMERO, 2008).

Nas referências Kane et al. (2000), Sanches e Coda (2013) e Coda e Paccola (2009), mostra-se que para formulações Lagrangeanas totais com matriz de massa constante pode-se utilizar o algoritmo de Newmark com suficiente precisão em análise de estruturas. Além desse motivo, com base nas conclusões de Siqueira (2016), utiliza-se o algoritmo de Newmark nesse trabalho, incluindo dinâmica de multicorpos nas aplicações. 


\subsection{Motivação e objetivos}

O trabalho tem como objetivo o estudo de estruturas com dispositivos FPS e TMD como sistemas de controle de vibração aplicando a formulação não linear geométrica pelo método dos elementos finitos posicional (MEFP). Dessa forma, ampliar as aplicações do método proposto pelo grupo de pesquisa de métodos numéricos do SET. Especificamente, objetiva-se a implementação computacional de elementos de chapa, barra geral, treliça e restrições cinemáticas via multiplicadores de Lagrange.

Como visto na revisão bibliográfica, a modelagem de estruturas com sistemas de controle estrutural aplicando formulações geometricamente não lineares não tem sido abordada com a devida frequência na literatura especializada, portanto, o presente trabalho pode ser um ponto de partida para a implementação de análises geometricamente exatas do problema além de fornecer exemplos de comparação com analises aproximadas atualmente disponíveis na literatura.

Além disso, busca-se incrementar a formação do bolsista no que diz respeito à implementação computacional e à modelagem dinâmica não linear de estruturas e mecanismos, incluindo o comportamento de dispositivos de controle de vibração.

\subsection{Metodologia}

De modo a alcançar os objetivos traçados, desenvolveu-se um código computacional empregando a formulação posicional do método dos elementos finitos para análise estática e dinâmica não linear geométrica de elementos tipo pórtico plano, solido bidimensional (Chapa) e treliça plana apresentadas nos trabalhos de Coda e Paccola (2014), Marques (2006) e Madeira e Coda (2016), respectivamente.

Seguidamente, introduziram-se as restrições cinemáticas via multiplicadores de Lagrange que permitem a simulação de ligações deslizantes entre elementos tipo pórtico como feito no trabalho de Siqueira (2016) e estendeu-se sua aplicação ao 
deslizamento entre elementos sólidos bidimensionais. Além disso, implementou-se a formulação específica apresentada em Madeira e Coda (2016) para a modelagem de dispositivos massa/mola/amortecedor.

Assim sendo, dispõe-se de um conjunto de ferramentas que permitem a modelagem numérica de estruturas planas com dispositivos FPS e TMD, onde a estrutura primaria representa-se com elementos tipo pórtico com cinemática de Reissner.

No que diz respeito à modelagem do FPS, utilizam-se elementos tipo chapa para discretizar o corpo de todos os componentes do dispositivo, isto é, a chapa superior, inferior e o deslizador. Empregam-se elementos tipo pórtico nas linhas do contorno dos elementos sólidos que se deslizam. Portanto, a rigor, o deslizamento ocorre entre elementos tipo pórtico que encarregam-se de transmitir as forças para os elementos sólidos bidimensionais (Ver figura 2).
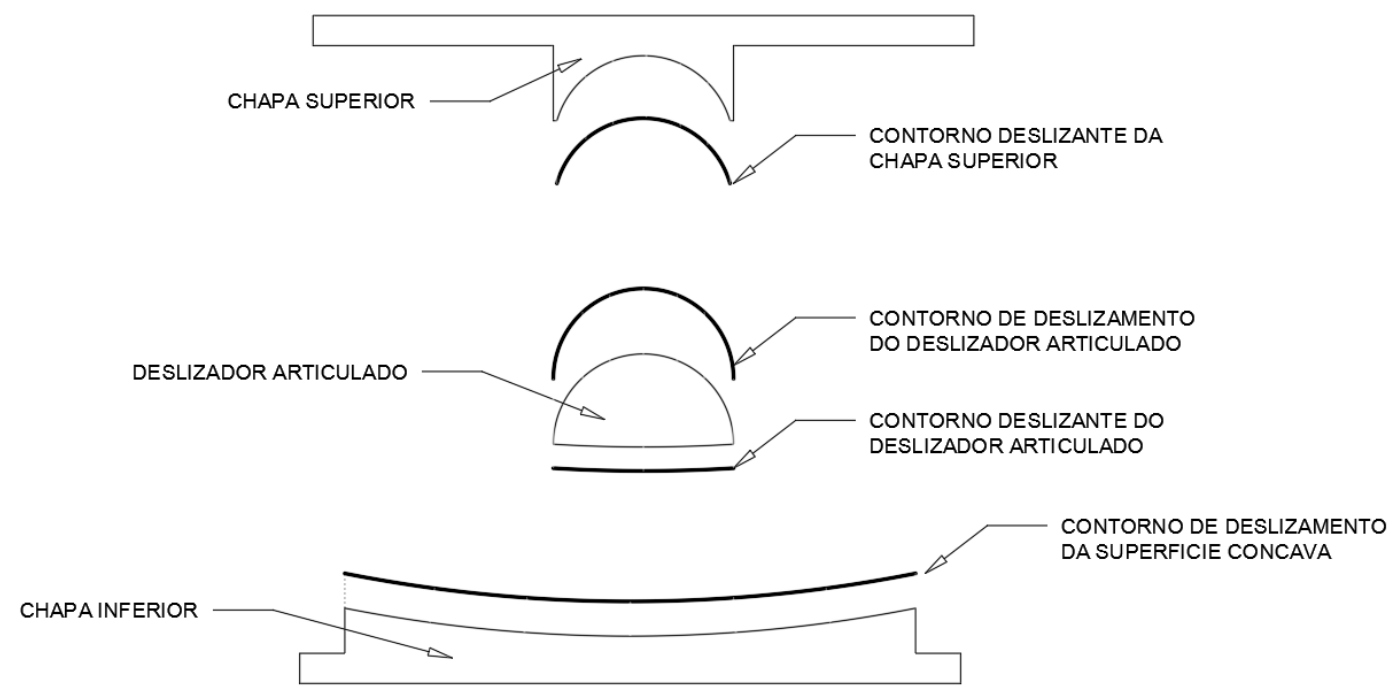

Figura 4 -Identificação dos componentes do dispositivo FPS para a modelagem.

Aos elementos pórtico pode ou não ser atribuída uma rigidez que represente as camadas de revestimento do sólido. Além disso, comenta-se que a formulação implementada representa uma condição de deslizamento liso, portanto, não leva em consideração o atrito que apesar de ser baixo, no caso de dispositivos FPS, existe. 
Para a modelagem do TMD emprega-se 0 dispositivo massa/mola/amortecedor instalado no topo da estrutura. O dispositivo consiste em duas barras de treliça alinhadas que seguram uma massa concentrada. Por sua vez, a cada barra de treliça atribui-se um valor de rigidez e de amortecimento viscoso. $\mathrm{O}$ alinhamento entre as barras garante-se por meio de multiplicadores de Lagrange.

No capítulo 4 encontra-se descrito, detalhadamente, a modelagem dos dispositivos descritos. 


\section{FORMULAÇÃO POSICIONAL DO MÉTODO DOS ELEMENTOS FINITOS PARA ANÁLISE NÃO LINEAR GEOMÉTRICA DE ESTRUTURAS}

Apesar das formulações aqui empregadas serem bem conhecidas no SET é importante retomá-las tendo em vista que estas foram implementadas integralmente para o desenvolvimento do programa de análise estrutural apresentado.

O desenvolvimento da formulação parte do funcional de energia total para sistemas isotérmicos escrito em função das posições nodais, em lugar dos deslocamentos como é feito na abordagem clássica do método dos elementos finitos. A seguir aplica-se o princípio da estacionariedade para obter a configuração de equilíbrio. O sistema de equações resultante é integrado no tempo pelo algoritmo de Newmark e a não linearidade do sistema é então resolvida pelo método incremental-iterativo de Newton-Raphson.

Na formulação implementada consideram-se forças conservativas. A seguir apresenta-se uma descrição breve dos elementos utilizados bem como de implentações específicas são mostradas neste capítulo.

\subsection{Elemento de treliça}

Uma forma simples de se introduzir o método dos elementos finitos posicional é a descrição do elemento finito de treliça plana que possui formas fechadas em seu desenvolvimento, dispensando técnicas de integração numérica. Além disso neste item se apresenta todo o processo de solução dinâmico não linear geométrico incluindo a formulação de um dispositivo de controle de vibração de estruturas do tipo amortecedor viscoso.

Partindo da energia total para sistemas isotermicos, dada por:

$$
\Pi=\mathbb{K}+\mathbb{Q}+U_{e}-\mathbb{P}
$$


Nesta, $\Pi$ designa o funcional de energia total, $\mathbb{K}$ é a energia cinética, $\mathbb{Q}$ é a dissipação de energia por amortecimento viscoso, $U_{e}$ denota a energia de deformação do corpo e $\mathbb{P}$ representa a energia potencial das forças externas.

Em seguida aplica-se o princípio da energia total estacionaria para a obtenção das equações do movimento.

$$
\delta \Pi=\frac{d \mathbb{K}}{d t} d t+\frac{\partial \mathbb{Q}}{\partial Y_{\alpha}^{\beta}} \delta Y_{\alpha}^{\beta}+\frac{\partial U e}{\partial Y_{\alpha}^{\beta}} \delta Y_{\alpha}^{\beta}-\frac{\partial P}{\partial Y_{\alpha}^{\beta}} \delta Y_{\alpha}^{\beta}=0
$$

Na qual, o termo $Y_{\alpha}^{\beta}$ refere-se à posição do nó $\beta$ na direção $\alpha$ e $t$ simboliza o tempo.

Tendo em vista que a equação [2.2] representa o equilíbrio de forças, os termos que a compõem correspondem à força inercial, à força de dissipação (modelo de amortecimento viscoso), à força interna e à força externa, respectivamente. Nos seguintes itens apresenta-se o cálculo de cada parcela da equação anterior.

\subsubsection{Calculo da força inercial e da força de amortecimento viscoso}

A energia cinética para um sistema continuo pode ser escrita como:

$$
\mathbb{K}=\frac{1}{2} \int_{V o} \rho_{o} \dot{Y}_{i} \dot{Y}_{i} d V o
$$

Sendo, $\rho_{0}$ e $V o$ à densidade do material e o volume das barras de treliça na configuração inicial, respectivamente, $\dot{Y}_{i}$ a velocidade na direção $i$ de um ponto genérico contido no domínio $V o$. Em relação à notação, é importante mencionar que o número de pontos em cima de um variável indica o grau da derivada em relação ao tempo.

Levando em consideração o princípio da conservação da massa, a variação da energia cinética fica dada por:

$$
\delta \mathbb{K}=\frac{d \mathbb{K}}{d t} d t=\int_{V o} \rho_{o} \ddot{Y}_{i} \dot{Y}_{i} d t d V o=\int_{V o} \rho_{o} \ddot{Y}_{i} \delta Y_{i} d V o
$$


$\mathrm{Na}$ equação anterior pode ser introduzida a aproximação da técnica dos elementos finitos para elementos de barra de aproximação linear o qual geraria a matriz de massa consistente. No presente trabalho, os elementos de treliça foram analisados com o modelo de massa concentrada, dessa forma a variação da energia cinética é expressa por:

$$
\delta \mathbb{K}=\sum_{e f=1}^{n f} \int_{V o} \rho_{o} d V o \ddot{Y}_{\alpha}^{\beta} \delta Y_{\alpha}^{\beta}=M^{(\beta)} \ddot{Y}_{\alpha}^{\beta} \delta Y_{\alpha}^{\beta}
$$

Onde $M^{(\beta)}$ representa a matriz de massa associada ao nó $\beta$ calculada como a metade da soma das massas dos elementos finitos conectados naquele nó além das massas concentradas sobrepostas e ef o índice que percorre o número de elementos finitos $(n f)$ da estrutura.

No que diz respeito à variação da energia de dissipação, no presente trabalho foi adotado o modelo de amortecimento de Rayleigh, também chamado amortecimento clássico. O modelo de amortecimento de Rayleigh expressa a matriz de amortecimento como uma combinação linear da massa e da matriz de rigidez, isto é:

$$
C=\alpha_{1} M+\alpha_{2} K
$$

$\mathrm{Na}$ qual, $\alpha_{1}$ e $\alpha_{2}$ são escalares, $C$ é a matriz de amortecimento e $K$ representa a matriz de rigidez. Nesse trabalho adotou-se a matriz de amortecimento proporcional à matriz de massa, de modo que foi empregado o modelo de Rayleigh com $\alpha_{2}=0$.

Finalmente, a parcela da variação da energia de dissipação é escrita como:

$$
\delta \mathbb{Q}=C \dot{Y}_{i}^{\beta} \delta Y_{i}^{\beta}
$$

\subsubsection{Cálculo das forças internas}

Observa-se da equação [2.2] que a variação da energia de deformação está associada às forças internas como resultado do conceito de conjugado energético. Portanto, para continuar com o desenvolvimento, é pertinente escrever a função que 
define a energia de deformação. A formulação empregada, adota como relação constitutiva o modelo mais empregado para análise elástica não linear geométrica de estruturas que apresentam grandes deslocamentos e pequenas deformações, isto é, o modelo de Saint-Venant-Kirchoff (SVK) que expressa uma relação linear, análoga à Lei de Hooke, entre a medida de deformação de Green-Lagrange $(E)$ e o segundo tensor de tensão de Piola-Kirchoff $(S)$. Para cada elemento finito do tipo treliça essa relação constitutiva pode ser escrita da seguinte forma:

$$
U e_{(e f)}=\int_{V o_{(e f)}} u_{e} d V o=\frac{1}{2} \mathfrak{c} E^{2} V o_{(e f)}
$$

Sendo, $u_{e}$ a energia especifica de deformação e c o modulo elástico que coincide com o módulo de Young para deformações infinitesimais. Observa-se que todas as grandezas são constates ao longo da barra, por tanto dispensa-se de uma técnica numérica para sua determinação.

A medida de deformação de Green-Lagrange uniaxial define-se como:

$$
\begin{gathered}
E=\frac{1}{2}\left(\frac{L^{2}-L o^{2}}{L o^{2}}\right) \\
L=\sqrt{\left(Y_{1}^{1}-Y_{1}^{2}\right)^{2}+\left(Y_{2}^{1}-Y_{2}^{2}\right)^{2}} \\
L o=\sqrt{\left(X_{1}^{1}-X_{1}^{2}\right)^{2}+\left(X_{2}^{1}-X_{2}^{2}\right)^{2}}
\end{gathered}
$$

$\mathrm{Na}$ qual $L$ e $L o$ denotam o comprimento na configuração atual e inicial, respectivamente, $Y$ representa a posição na configuração atual e $X$ a posição na configuração inicial. $O$ índice sobrescrito define o nó do elemento na numeração local e o índice subscrito denota a direção no plano.

Agora é possível definir a parcela da variação da energia de deformação da seguinte forma:

$$
\delta U e=\sum_{e f=1}^{n f} \mathfrak{C} E A o L o \frac{\partial E}{\partial Y_{\alpha}^{\beta}} \delta Y_{\alpha}^{\beta}
$$

Sendo $A o$ a área da seção transversal da barra de treliça na configuração inicial. A derivada do tensor de deformação de Green-Lagrange em relação aos parâmetros nodais determina-se como: 


$$
\frac{\partial E}{\partial Y_{\alpha}^{\beta}}=\frac{(-1)^{\beta}}{L o^{2}}\left(Y_{\alpha}^{2}-Y_{\alpha}^{1}\right)
$$

\subsubsection{Estratégia de solução}

Substituindo as equações [2.5], [2.7] e [2.11] na equação [2.2] e incluindo a variação do potencial das forças externas para o caso de forças conservativas, obtém-se a seguinte expressão:

$$
\delta \Pi=\sum_{e f=1}^{n f}\left[M \ddot{Y}_{\alpha}^{\beta}+C \dot{Y}_{\alpha}^{\beta}+\subseteq E A o L o \frac{\partial E}{\partial Y_{\alpha}^{\beta}}-F_{\alpha}^{\beta}\right] \delta Y_{\alpha}^{\beta}=0
$$

$\mathrm{Na}$ qual, devido à arbitrariedade da variação $\delta Y_{\alpha}^{\beta}$ obtém-se a equação de equilíbrio dinâmico que pode ser reescrita em notação diádica como:

$$
\frac{\partial \Pi}{\partial \vec{Y}}=M \cdot \ddot{\vec{Y}}+C \cdot \dot{\vec{Y}}+\vec{F} \text { int }-\vec{F} \text { ext }=\overrightarrow{0}
$$

A estratégia de solução para resolver o sistema de equações [2.14] combina o algoritmo de integração temporal de Newmark com o método aberto-incrementaliterativo de Newton-Raphson. À vista disso, a seguir apresentam-se as aproximações de Newmark, onde 3 e $\gamma$ são parâmetros da formula e os indices $s$ e $s+1$ designam o instante de tempo passado e atual, respectivamente.

$$
\begin{gathered}
Y_{S+1}=Y_{S}+\Delta t \dot{Y}_{S}+\Delta t^{2}\left(\left(\frac{1}{2}-\mathcal{B}\right) \ddot{Y}_{S}+\mathcal{B} \ddot{Y}_{S+1}\right) \\
\dot{Y}_{S+1}=\dot{Y}_{S}+\Delta t(1-\gamma) \dot{Y}_{S}+\gamma \Delta t \ddot{Y}_{S+1}
\end{gathered}
$$

Dependendo dos valores adotados para os parâmetros 3 e $\gamma$ determina-se a característica dissipação de energia e a estabilidade do método e $\Delta t$ refere-se à discretização temporal. Vários autores têm compilado e discutido as características dos métodos de integração temporal, por exemplo, PAULTRE (2010). No presente trabalho, foi implementado o método da aceleração média $\left(\gamma=\frac{1}{2}\right.$ e $\left.3=\frac{1}{4}\right)$.

Substituindo as equações [2.15] na equação de movimento [2.14] encontrase: 


$$
\begin{aligned}
& \left.\frac{\partial \Pi}{\partial \vec{Y}}\right|_{s+1}=\vec{F} \text { int }_{s+1}-\vec{F} \text { ext }_{s+1}+\frac{M}{\mathcal{G} \Delta t^{2}} \cdot \vec{Y}_{s+1}+\frac{\gamma C}{\mathcal{G} \Delta t} \cdot \vec{Y}_{s+1}-M \cdot \vec{Q}_{s}+C \cdot \vec{R}_{s} \\
& -\gamma \Delta t C \cdot \vec{Q}_{s}=\overrightarrow{0}
\end{aligned}
$$

Onde $Q_{s}$ e $R_{S}$ representam as contribuições do instante de tempo passado e são determinadas por:

$$
\begin{gathered}
\vec{Q}_{s}=\frac{\vec{Y}_{s}}{\mathcal{B} \Delta t^{2}}+\frac{\dot{\vec{Y}}_{s}}{\mathcal{B} t}+\left(\frac{1}{2 \mathcal{B}}-1\right) \ddot{\vec{Y}}_{s} \\
\vec{R}_{s}=\dot{\vec{Y}}_{s}+\Delta t(1-\gamma) \ddot{\vec{Y}}_{s}
\end{gathered}
$$

Percebe-se que a equação de equilíbrio dinâmico [2.16] é uma equação não linear com respeito às posições no instante atual $\left(\vec{Y}_{s+1}\right)$. Além disso, pode ser entendida como uma função do vetor posição $\left(\vec{g}_{s+1}\left(\vec{Y}_{s+1}\right)=\overrightarrow{0}\right)$ para facilitar o seu entendimento ao expandi-la em série de Taylor truncada em primeira ordem conforme segue:

$$
\overrightarrow{0}=\vec{g}\left(\vec{y}_{s+1}\right) \cong \vec{g}\left({\overrightarrow{y_{0}}}_{s+1}\right)+\nabla \vec{g}\left({\overrightarrow{y_{0}}}_{s+1}\right) \cdot \Delta Y
$$

$\mathrm{Na}$ qual, ${\overrightarrow{y_{0}}}_{s+1}$ representa a posição tentativa para o cálculo de $\vec{Y}_{s+1}$ e portanto o vetor $\vec{g}$ corresponde ao vetor de desbalanceamento mecânico do sistema quando a posição tentativa ${\overrightarrow{y_{0}}}_{s+1}$ não coincide com o vetor posição correto $\vec{Y}_{s+1}$. O gradiente do vetor de desbalanceamento mecânico $\nabla \vec{g}$ representa a matriz hessiana $H$ do funcional de energia total dada por:

$$
\begin{aligned}
H=\left.\frac{\partial^{2} \Pi}{\partial \vec{Y}^{2}}\right|_{s+1} & =\nabla \vec{g}\left({\overrightarrow{y_{0}}}_{s+1}\right)=\left.\frac{\partial^{2} U e}{\partial \vec{Y}^{2}}\right|_{s+1}+\frac{M}{\mathcal{B} \Delta t^{2}}+\frac{\gamma C}{\mathcal{B} \Delta t} \\
& =\left(H^{\text {estatica }}+\frac{M}{\mathcal{B} \Delta t^{2}}+\frac{\gamma C}{\mathcal{B} \Delta t}\right)
\end{aligned}
$$

Onde o termo $\left.\frac{\partial^{2} U e}{\partial \vec{Y}^{2}}\right|_{S+1}$ é chamado de matriz hessiana estática ( $\left.H^{\text {estatica }}\right)$ e está dada por:

$$
H_{\alpha \beta z \theta}^{\text {estatica }}=\sum_{e f=1}^{n f}\left(\mathfrak{C} A o L o \frac{\partial E}{\partial Y_{Z}^{\theta}} \frac{\partial E}{\partial Y_{\alpha}^{\beta}}+\mathfrak{C} E A o L o \frac{\partial^{2} E}{\partial Y_{\alpha}^{\beta} \partial Y_{Z}^{\theta}}\right)
$$

Sendo: 


$$
\frac{\partial^{2} E}{\partial Y_{\alpha}^{\beta} \partial Y_{z}^{\theta}}=\frac{\delta_{\alpha z}(-1)^{\beta}(-1)^{\theta}}{L o^{2}}
$$

Onde os índices $z$ e $\theta$ representam a direção e o nó, respectivamente, de forma análoga aos índices $\alpha$ e $\beta$.

Da expressão [2.18] obtém-se o sistema de equações linear utilizado para calcular a correção do vetor posição tentativa, isto é:

$$
0=\vec{g}\left(\overrightarrow{y_{0}}\right)+\left.\frac{\partial \vec{g}}{\partial \vec{y}}\right|_{\overrightarrow{y_{0}}} \cdot \overrightarrow{\Delta Y} \rightarrow \overrightarrow{\Delta Y}=-H^{-1} * \vec{g}\left(\overrightarrow{y_{0}}\right)
$$

Resolvendo a equação [2.22] para obter a correção $\Delta Y$ é possível recalcular o vetor tentativa como segue:

$$
\vec{Y}_{s+1}={\overrightarrow{y_{0}+1}}+\overrightarrow{\Delta Y}
$$

Assim sendo, o vetor posição tentativa ${\overrightarrow{y_{0}}}_{s+1}$ é corrigido iterativamente dentro de cada passo de tempo e para o seguinte passo de tempo adota-se como primeira tentativa o vetor posição do passo de tempo anterior.

Em seguida é recalculada a aceleração para cada iteração por:

$$
\ddot{\vec{Y}}_{s+1}=\frac{\vec{Y}_{s+1}}{\mathcal{B} \Delta t^{2}}-\vec{Q} s
$$

O critério de convergência do processo iterativo pode ser adotado em termos de força ou posição segundo as expressões abaixo:

$$
\left\|\vec{g}\left(\overrightarrow{y_{0}}\right)\right\| \leq \text { Tol ou }\|\overrightarrow{\Delta Y}\| /\|\vec{X}\| \leq \text { Tol }
$$

Sendo Tol o valor de tolerância predefinido. É importante ressaltar que os vetores $\vec{Q} s$ e $\vec{R}_{s}$ somente serão atualizados quando o critério de convergência, no instante de tempo atual, for atendido. Ainda destaca-se que no primeiro passo de tempo a aceleração determina-se como:

$$
\ddot{\vec{Y}}_{o}=M^{-1}\left(F o-\left.\frac{\partial U e}{\partial Y}\right|_{o}-C \dot{Y}_{o}\right)
$$


Onde o índice subscrito " $o$ " denota $\mathrm{o}$ instante de tempo em que a análise dinâmica inicia.

\subsubsection{Modelo de amortecimento Kelvin/Voight modificado}

O modelo constitutivo de Kelvin que emprega a deformação de Green foi proposto por Madeira e Coda (2016) e chamado de modelo Kelvin/Voight modificado. Neste item apresenta-se brevemente o seu desenvolvimento.

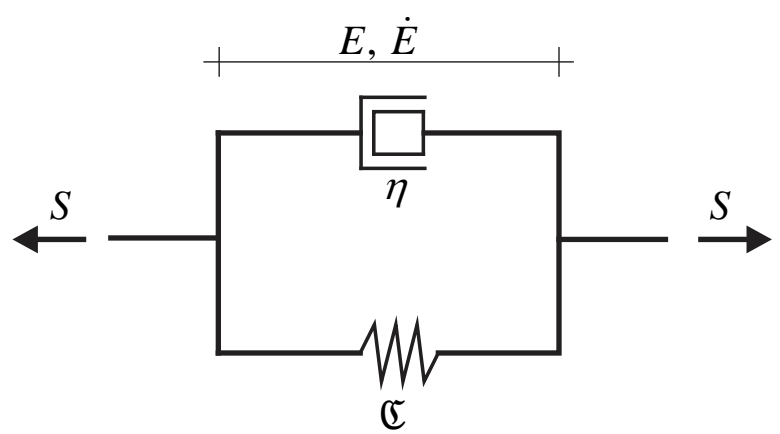

Figura 5 - Modelo Kelvin/Voight modificado. Tomado de Madeira e Coda (2016)

Segundo a figura, a segunda tensão de Piola-Kirchoff é função da deformação de Green $E$, da velocidade de deformação $\dot{E}$, da constante elástica do material $\mathfrak{C}$ e da viscosidade dinâmica $\eta$ conforme segue:

$$
S=\mathfrak{C} E+\eta \dot{E}
$$

Onde o primeiro termo é a derivada da energia específica de deformação e o segundo termo corresponde à parcela associada à dissipação de energia do modelo viscoso (Q). Para o elemento finito de treliça, a variação do potencial de dissipação em relação aos parâmetros nodais na formulação posicional, pode ser escrita como:

$$
\delta \mathrm{Q}_{(e f)}=\eta \dot{E} A o L o \frac{(-1)^{\beta}}{L o^{2}}\left(Y_{\alpha}^{2}-Y_{\alpha}^{1}\right) \delta Y_{\alpha}^{\beta}=F_{\alpha}^{\beta(v i s)} \delta Y_{\alpha}^{\beta}
$$


O cálculo da velocidade de deformação obtém-se aplicando o método das diferencias finitas e considerando que o passo de tempo é o suficientemente pequeno para assumir $\left(Y_{\alpha}^{2}-Y_{\alpha}^{1}\right)_{s} \approx\left(Y_{\alpha}^{2}-Y_{\alpha}^{1}\right)_{s+1}$, dessa forma, determina-se como:

$$
\left(F_{\alpha}^{\beta(v i s)}\right)_{e f}=\frac{\eta}{\mathbb{C} \Delta t}\left[\left(F_{\alpha(s+1)}^{\beta(\text { interna })}\right)_{e f}-\left(F_{\alpha(s)}^{\beta(\text { interna })}\right)_{e f}\right]
$$

Logo, a força interna viscosa esta numericamente relacionada à força interna elástica.

Levando em consideração a variação da energia de dissipação e aproveitando a arbitrariedade da variação $\delta Y_{\alpha}^{\beta}$ a equação de equilíbrio dinâmico fica expressa por:

$$
\vec{g}=\frac{\partial \Pi}{\partial \vec{Y}}=M \cdot \ddot{\vec{Y}}+C \cdot \dot{\vec{Y}}+\vec{F} \text { int }+\vec{F} v i s-\vec{F} \text { ext }=\overrightarrow{0}
$$

Reescrevendo a equação [2.30] para o instante atual $s+1$ e substituindo a expressão dada para a força viscosa, chega-se a:

$$
\vec{g}_{s+1}=M \cdot \ddot{\vec{Y}}_{s+1}+C \cdot \dot{\vec{Y}}_{s+1}+\left(1+\frac{\eta}{\mathbb{C} \Delta t}\right) \vec{F}_{s+1}^{i n t}-\frac{\eta}{\mathfrak{C} \Delta t} \vec{F}_{s}^{\text {int }}-\vec{F}_{s+1}^{\text {ext }}=\overrightarrow{0}
$$

Introduzindo as aproximações de Newmark, encontra-se:

$$
\begin{gathered}
\vec{g}_{s+1}=\left(1+\frac{\eta}{\mathfrak{C} \Delta t}\right) \vec{F}_{s+1}^{i n t}-\frac{\eta}{\mathfrak{C} \Delta t} \vec{F}_{s}^{i n t}-\vec{F} \text { ext } t_{s+1}+\frac{M}{\mathcal{B} \Delta t^{2}} \cdot \vec{Y}_{S+1}+\frac{\gamma C}{\mathcal{B} \Delta t} \cdot \vec{Y}_{S+1} \\
-M \cdot \vec{Q}_{s}+C \cdot \vec{R}_{s}-\gamma \Delta t C \cdot \vec{Q}_{s}=\overrightarrow{0}
\end{gathered}
$$

Observa-se que a equação [2.32] é similar à equação [2.16] já que também é um sistema não linear respeito às posições $\vec{Y}_{s+1}$. Desse modo, pode ser aplicado o mesmo algoritmo de solução descrito no item anterior nas equações [2.22] até [2.26]. No entanto, para levar em consideração o modelo viscoso apresentado, o vetor de desbalanceamento mecânico deve ser calculado segundo a equação [2.32] e a matriz hessiana segundo a seguinte expressão:

$$
\begin{aligned}
H=\left.\frac{\partial^{2} \Pi}{\partial \vec{Y}^{2}}\right|_{s+1} & =\nabla \vec{g}\left({\overrightarrow{y_{0}+1}}_{s}\right)=\left.\frac{\partial^{2}(U e+Q)}{\partial \vec{Y}^{2}}\right|_{s+1}+\frac{M}{\mathcal{B} \Delta t^{2}}+\frac{\gamma C}{\mathcal{B} \Delta t} \\
& =\left(\left(1+\frac{\eta}{\mathfrak{C} \Delta t}\right) H^{\text {estatica }}+\frac{M}{\mathcal{B} \Delta t^{2}}+\frac{\gamma C}{\mathcal{B} \Delta t}\right)
\end{aligned}
$$


Apesar da formulação aqui apresentada ser aplicável a qualquer elemento finito, é de interesse desenvolver a formulação específica para simular o dispositivo passivo massa/mola/amortecedor empregado como sistema de controle de vibração. No seguinte item mostra-se seu desenvolvimento.

\subsubsection{Dispositivo massa/mola/amortecedor}

Como mencionado no capítulo 1 , os sistemas de controle de vibração de estruturas classificam-se segundo o seu comportamento. Aqueles dispositivos que não precisam de uma fonte externa de potência para o seu funcionamento são chamados de passivos. Dentro desse grupo encontra-se o dispositivo implementado nesse trabalho.

$\mathrm{Na}$ figura 6 apresenta-se um esquema do dispositivo massa/mola/amortecedor, chamado $z$, conformado por duas barras de treliça, definidas pelos nós $k, l$ e $r$, com rigidez elástica $(K)$ e viscosidade dinâmica $(\eta)$ e com uma massa concentrada de valor $m$ no nó comum. Lembra-se que as coordenadas $x_{1}$ e $x_{2}$ representam a posição horizontal e vertical, respectivamente, do corpo na configuração inicial. De forma análoga, $y_{1}$ e $y_{2}$ representam a posição na configuração atual do corpo.

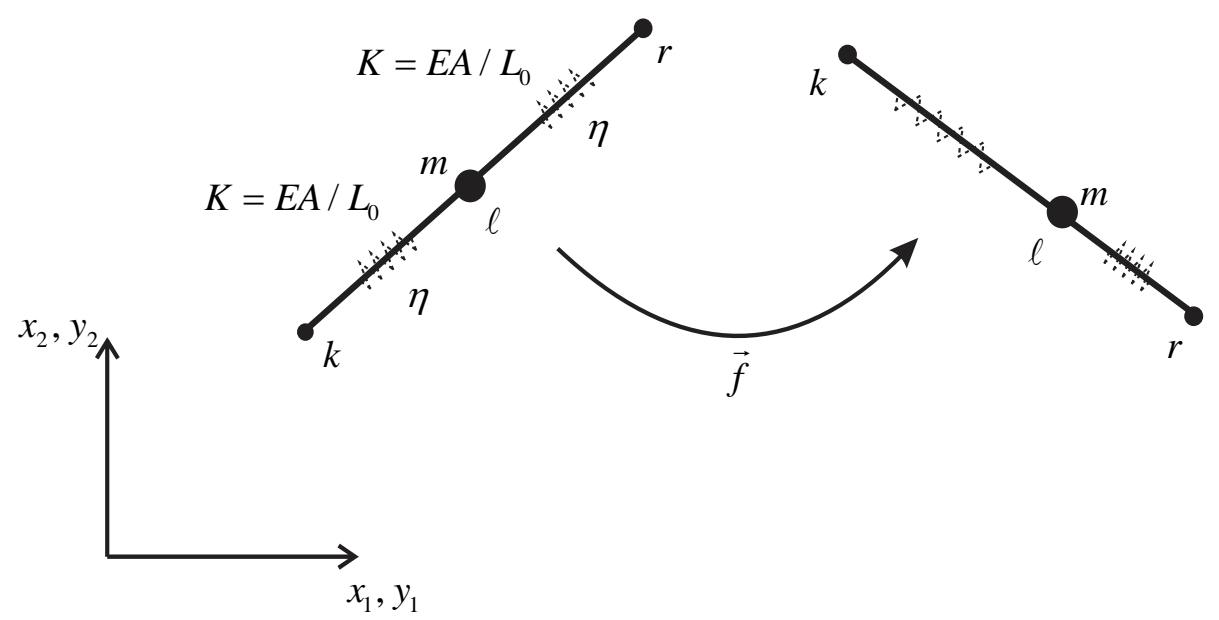

Figura 6 - Dispositivo massa/mola/amortecedor. Tomado de Madeira e Coda (2016). 
Devido à geometria do dispositivo e tendo em vista que o elemento de treliça não suporta cargas transversais ao eixo da barra é necessário impor uma restrição cinemática que garanta que o deslocamento relativo entre os nós seja puramente longitudinal. Para o caso bidimensional, essa restrição cinemática é dada por:

$$
\left(Y_{2}^{r}-Y_{2}^{l}\right)\left(Y_{1}^{r}-Y_{1}^{k}\right)-\left(Y_{2}^{r}-Y_{2}^{k}\right)\left(Y_{1}^{r}-Y_{1}^{l}\right)=0
$$

A restrição cinemática é introduzida no funcional de energia total somando o potencial Lagrangeano (L) a seguir:

$$
\underline{\mathrm{L}}=\sum_{z} \mathrm{~L}_{z}=\sum_{z} \lambda^{z}\left[\left(Y_{2}^{r}-Y_{2}^{l}\right)\left(Y_{1}^{r}-Y_{1}^{k}\right)-\left(Y_{2}^{r}-Y_{2}^{k}\right)\left(Y_{1}^{r}-Y_{1}^{l}\right)\right]
$$

Onde o somatório varia de 1 até o número de dispositivos instalados. Dessa forma a energia total do sistema e sua variação fica dada pelas expressões [2.36] e [2.37], respectivamente.

$$
\begin{gathered}
\Pi=\mathbb{K}+\mathbb{Q}+U_{e}-\mathbb{P}+\underline{\mathrm{L}} \\
\delta \Pi=\delta \mathbb{K}+\delta \mathbb{Q}+\delta U_{e}-\delta \mathbb{P}+\delta \underline{L}=0
\end{gathered}
$$

Nota-se que os multiplicadores de Lagrange também são variáveis livres, portanto a variação da restrição cinemática L escreve-se como segue:

$$
\delta \underline{\mathrm{L}}=\frac{\partial \underline{\mathrm{L}}}{\partial Y_{\alpha}^{\beta}} \delta Y_{\alpha}^{\beta}+\frac{\partial \underline{\mathrm{L}}}{\partial \lambda_{j}^{Z}} \delta \lambda_{j}^{Z}=\hat{F}_{\alpha}^{\beta} \delta Y_{\alpha}^{\beta}+\Lambda_{j}^{Z} \delta \lambda_{j}^{Z}
$$

Onde $\hat{F}_{\alpha}^{\beta}$ refere-se à força devida à restrição cinemática na direção $\alpha$ quando o nó ativo $\beta$ pertença ao dispositivo $z$ e $\lambda_{j}^{z}$ são os multiplicadores de Lagrange associados. No apêndice do trabalho de Madeira e Coda (2016) apresenta-se o cálculo detalhado do vetor $\hat{F}_{\alpha}^{\beta}$ e $\Lambda_{j}^{z}$.

De forma análoga como foi mostrado no item 2.1.3 devido à arbitrariedade da variação da posição $\delta Y_{\alpha}^{\beta}$ e da variação dos multiplicadores $\delta \lambda_{j}^{Z}$ associados ao dispositivo $z$, chega-se à equação de equilíbrio dinâmico:

$$
\left(\hat{F}_{\alpha}^{\beta}+F_{\alpha}^{\beta(i n t)}\right)+F_{\alpha}^{\beta(v i s)}+M \cdot \ddot{Y}_{\alpha}^{\beta}+C \cdot \dot{Y}_{\alpha}^{\beta}-F_{\alpha}^{\beta(e x t)}=0
$$


E às equações que contém as restrições cinemáticas:

$$
\Lambda_{j}^{Z}=0_{j}^{Z}
$$

Dessa forma, o fato de ter introduzido as restrições cinemáticas via multiplicadores de Lagrange acrescentou o número de variáveis e equações a considerar. Para o caso bidimensional, o número de equações a mais é igual ao número de dispositivos instalados $n z$.

O algoritmo de solução é idêntico àqueles apresentados nos itens $2.1 .3 \mathrm{e}$ 2.1.4 mas modificando o vetor de desbalanceamento mecânico e a matriz hessiana segundo o mostrado no presente item. Para o caso bidimensional, os termos a serem adicionados na matriz hessiana são dados por:

$$
H_{z}=\left[\begin{array}{cc}
\frac{\partial^{2} L_{z}}{\partial Y_{\alpha}^{\beta} \partial Y_{\gamma}^{\theta}} & \frac{\partial^{2} L_{z}}{\partial Y_{\alpha}^{\beta} \partial \lambda_{j}^{(z)}} \\
\frac{\partial^{2} L_{z}}{\partial \lambda_{i}^{(z)} \partial Y_{\gamma}^{\theta}} & \frac{\partial^{2} L_{z}}{\partial \lambda_{i}^{(z)} \partial \lambda_{j}^{(z)}}
\end{array}\right]
$$

Onde os termos representados pela segunda linha e coluna da equação [2.41] correspondem aos graus de liberdade adicionados pela inclusão do dispositivo massa/mola/amortecedor. Os outros termos são adicionados nas posições existentes da matriz hessiana original. Em Madeira e Coda (2016) mostra-se em detalhe os termos que compõem a matriz $H_{z}$.

O algoritmo de solução do sistema não linear mostrado nos itens anteriores também aplica-se para resolver o sistema de equações aqui mostrado. Deve-se levar em conta que tanto o vetor solução $\vec{Y}$ quanto o vetor correção $\overrightarrow{\Delta Y}$ contêm a atualização dos multiplicadores de Lagrange para não inclui-los no cálculo do critério de convergência dado pelas equações [2.25].

Os itens até agora apresentados nesse capítulo mostram o algoritmo de solução base para resolver sistemas dinâmicos considerando a não linearidade geométrica pelo método dos elementos finitos posicional. Ainda foi apresentada a formulação do dispositivo massa/mola/amortecedor para controle de vibração de estruturas, na qual é importante destacar a imposição de restrições cinemáticas via 
multiplicadores de Lagrange dado que no capítulo 3 essa técnica será empregada para o desenvolvimento de ligações deslizantes.

\subsubsection{Exemplo 1 - Treliça de Mises}

Com o objetivo validar o código computacional gerado a partir da formulação detalhada nos itens anteriores, apresenta-se o exemplo descrito em Crisfield (1991) que consiste na obtenção da trajetória de equilíbrio para a treliça de Von-Mises aplicando deslocamento controlado no nó livre.

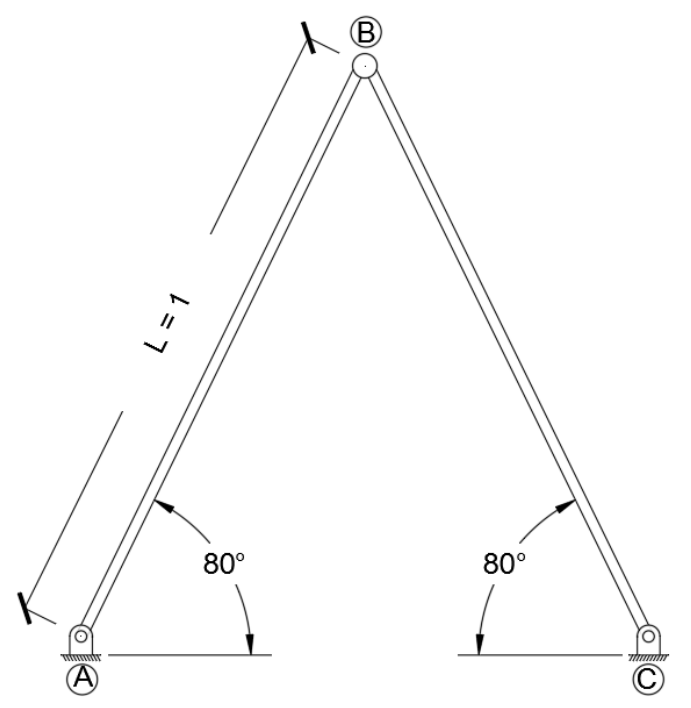

Figura 7 - Treliça de Von Mises

Na seguinte tabela são apresentados os dados de entrada.

\begin{tabular}{|lr|}
\hline \multicolumn{2}{|c|}{ Dados de entrada } \\
Area & 1 \\
Modulo de Young & $1 \mathrm{E}+07$ \\
Passos de carga & 1000 \\
\hline
\end{tabular}

Tabela 1 - Dados de entrada para análise da treliça de Von Mises. 
$\mathrm{Na}$ figura 8 plota-se o deslocamento vertical imposto no nó B e os valores correspondentes das forças que equilibram o sistema.

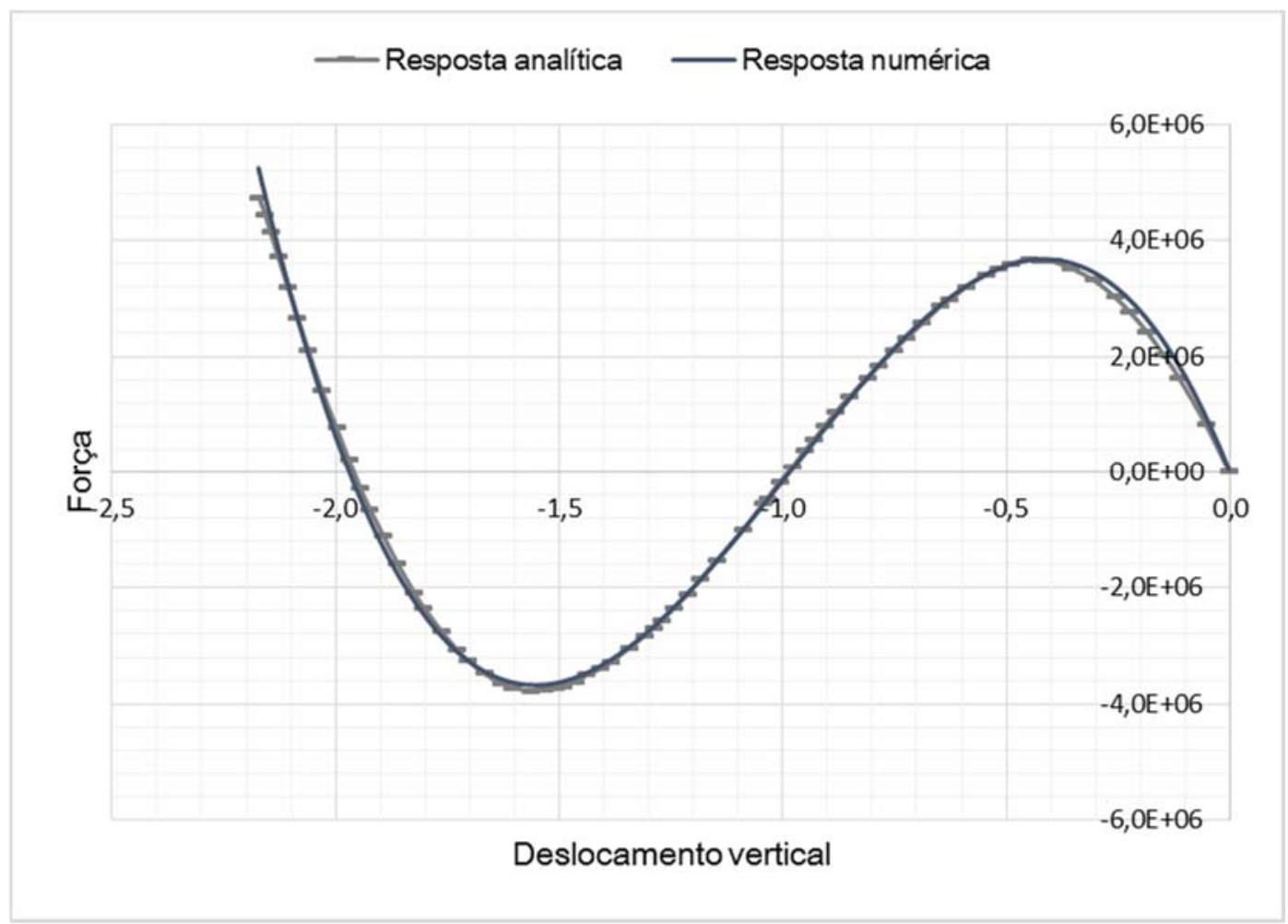

Figura 8 - Trajetória de equilíbrio do nó B da treliça de Von Mises.

2.1.7 Exemplo 2 - Validação do dispositivo massa/mola/amortecedor.

Esse exemplo apresenta a validação da formulação implementada para análise estática, quase-estática e dinâmica do dispositivo massa/mola/amortecedor. A figura 9 mostra a estrutura analisada com as seguintes propriedades mecânicas e geométricas: $\mathfrak{C}=10 \mathrm{Gpa}, A o=5 \mathrm{~cm}^{2}$ e $L o=1 \mathrm{~m}$ submetida a uma força $F=$ $10 \sqrt{2} k N$. 


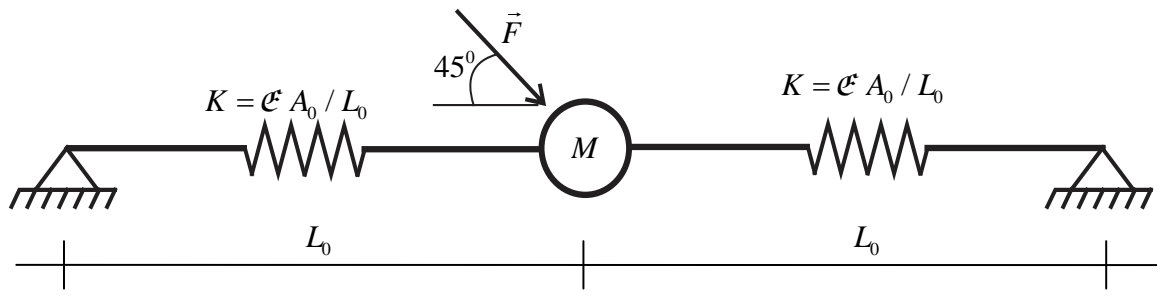

Figura 9 - Geometria do exemplo de validação dispositivo massa/mola/amortecedor. Tomado de Madeira e Coda (2016).

Para os valores dados, calcula-se a rigidez axial $K=\frac{5 M N}{m}$ e aplicando a lei de Hooke, obtém-se um deslocamento horizontal de $u_{h}=0.001 \mathrm{~m}$. Trata-se de um deslocamento pequeno, por tanto espera-se que o deslocamento obtido com 0 código computacional implementado seja praticamente o mesmo valor. Pela mesma razão, espera-se que em uma análise quase-estática o comportamento da estrutura se aproxime ao problema linear. Dessa forma, o deslocamento horizontal pode ser comparado com a resposta obtida da seguinte expressão:

$$
u_{h}=\left(\frac{\sqrt{2}}{2}\right) \cdot F \cdot L o \cdot\left(1-e^{-\frac{\varpi t}{\eta}}\right) /(2 \mathfrak{C} A o)
$$

Adotando $\eta=4 \mathrm{Mpa} \cdot s$, a figura 10 apresenta os resultados da análise quaseestática para diferentes valores de delta de tempo, mostrando que a formulação converge para a resposta obtida pela equação anterior. Além disso, observa-se que devido à restrição cinemática imposta, a massa concentrada somente apresenta deslocamento horizontal, enquanto a componente vertical da força aplicada é transmitida aos vínculos externos do dispositivo. 


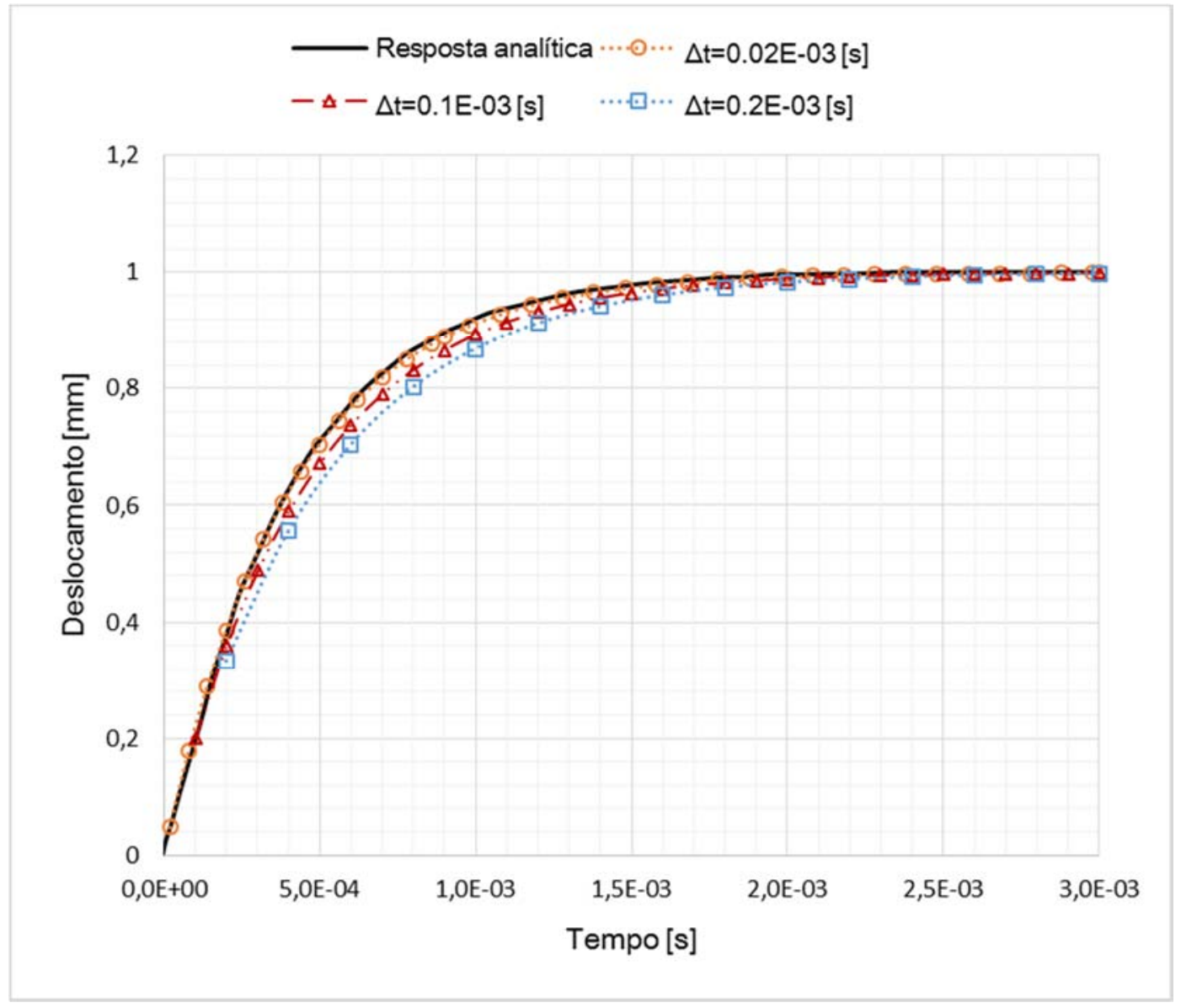

Figura 10 - Resposta quase-estática do dispositivo. 


\subsection{Elemento solido bidimensional}

Tendo em vista que a técnica de solução dinâmica não linear é a mesma para qualquer elemento finito (Combinação do método de integração temporal de Newmark com o método de Newton-Raphson) neste capítulo apresenta-se o desenvolvimento matemático para a obtenção da matriz de massa, o vetor de forças internas e a matriz hessiana do elemento de chapa. A grande diferença respeito à formulação apresentada para elementos de treliça é a inexistência de fórmulas fechadas e, portanto, é imprescindível o uso de técnicas de integração numérica.

A formulação implementada para elementos tipo chapa, descrita a seguir, encontra-se no trabalho de Marques (2006).

Na figura 11 mostra-se o corpo na configuração inicial Bo e na configuração atual $\mathrm{B}$, descritas por meio das funções de mapeamento $\vec{f}^{o}$ e $\vec{f}^{1}$, respectivamente. Percebe-se ainda que foi adotado um elemento finito triangular de dez nós (aproximação cubica), portanto, as integrais numéricas a serem avaliadas serão feitas aplicando a quadratura de Hammer.

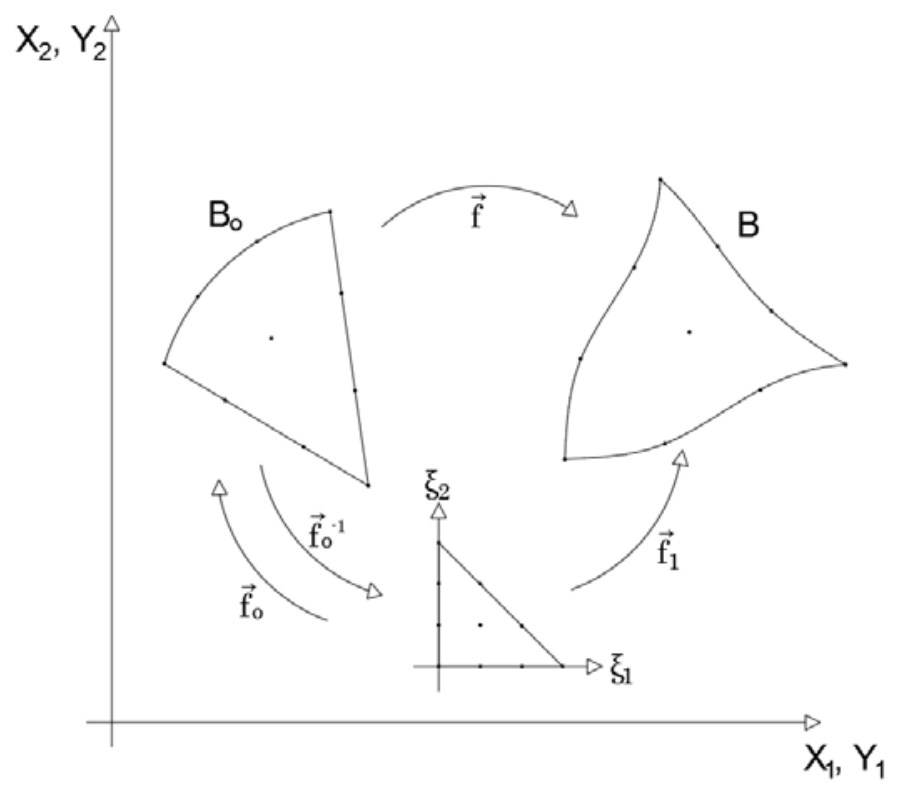

Figura 11 - Mapeamento das configurações inicial e atual e atual e sua representação no espaço adimensional. 
Introduzindo as aproximações do método dos elementos finitos posicional, expressas por:

$$
\begin{aligned}
& f_{i}^{0}\left(\xi_{1}, \xi_{2}\right)=X_{i}\left(\xi_{1}, \xi_{2}\right)=\phi_{l}\left(\xi_{1}, \xi_{2}\right) X_{i}^{l} \\
& f_{i}^{1}\left(\xi_{1}, \xi_{2}\right)=Y_{i}\left(\xi_{1}, \xi_{2}\right)=\phi_{l}\left(\xi_{1}, \xi_{2}\right) Y_{i}^{l}
\end{aligned}
$$

Onde $X_{i}^{l}$ e $Y_{i}^{l}$ denotam a posição do nó $l$ na direção $i$ na configuração inicial e final, respectivamente, e $\phi_{l}\left(\xi_{1}, \xi_{2}\right)$ designa a função de forma associada ao nó $l$ escrita em função das coordenadas adimensionais $\xi_{1}$ e $\xi_{2}$. Após ter definido as funções de mapeamento é possível escrever a função mudança de configuração $\vec{f}$ como segue:

$$
\vec{f}=\vec{f}^{1} \circ\left(\vec{f}^{o}\right)^{-1}
$$

E o seu gradiente como:

$$
A=A^{1} \cdot\left(A^{o}\right)^{-1}
$$

Sendo $A^{1}$ e $A^{o}$ dados por:

$$
\begin{aligned}
A_{i j}^{1} & =f_{i, j}^{1}=\phi_{l, j} Y_{i}^{l} \\
A_{i j}^{0} & =f_{i, j}^{0}=\phi_{l, j} X_{i}^{l}
\end{aligned}
$$

$\mathrm{Na}$ qual $\phi_{l, j}$ representa a derivada da função de forma associada ao nó $l$ em relação à variável adimensional $\xi$ na direção $j$. Tendo definido o gradiente da função mudança de configuração, calcula-se o tensor de deformação de Green $(E)$ e o tensor de alongamento de Cauchy-Green $(\mathcal{C})$ como:

$$
\begin{gathered}
E=\frac{1}{2}\left(A^{t} \cdot A-I\right) \\
\mathcal{C}=A^{t} \cdot A
\end{gathered}
$$

Sendo $I$ o tensor identidade de tamanho $2 \times 2$.

\subsubsection{Forças internas do elemento tipo chapa}

O vetor de forças internas do sistema é determinado a partir do conceito do conjugado energético que pode ser escrito como segue: 


$$
F_{\alpha}^{\beta(i n t)}=\frac{\partial U e}{\partial Y_{\alpha}^{\beta}}=\frac{\partial}{\partial Y_{\alpha}^{\beta}}\left(\sum_{e f=1}^{n f} \int_{V o} u_{e} d V o\right)
$$

Dessa expressão entende-se que a força é o conjugado energético da posição.

À vista disto, deve ser definida a energia especifica de deformação para o elemento solido bidimensional. No presente trabalho foi adotada a lei constitutiva de Saint-Venant-Kirchoff que escrita na sua forma integral para o estado plano de deformações fica dada por:

$$
u_{e}=\frac{1}{2}\left\{(1-v) K\left(E_{11}^{2}+E_{22}^{2}\right)+2 K v\left(E_{11} E_{22}\right)+2 \mathbb{G}\left(E_{12}^{2}+E_{21}^{2}\right)\right\}
$$

E para análise no estado plano de tensões:

$$
u_{e}=\frac{\mathbb{G}}{(1-v)}\left\{E_{11}^{2}+E_{22}^{2}+2 v\left(E_{11} E_{22}\right)+(1-v)\left(E_{12}^{2}+E_{21}^{2}\right)\right\}
$$

Nas quais, $E_{i j}$ refere-se ao valor na posição $i, j$ do tensor deformação de Green, $v$ representa o coeficiente de Poisson. Finalmente, $K$ e $\mathbb{G}$ são constantes elásticas do material dadas por:

$$
K=\frac{\mathbb{E}}{(1+v)(1-2 v)} \quad \mathbb{G}=\frac{\mathbb{E}}{2(1+v)}
$$

Onde o modulo de Young está representado por $\mathbb{E}$. Comenta-se ainda que nos desenvolvimentos subsequentes emprega-se a energia específica para o estado plano de deformações, ficando para o leitor a dedução do equacionamento do estado plano de tensões.

Conforme as equações [2.48] e [2.49] $u_{e}$ é função da deformação de Green $E$ que por sua vez relaciona-se com as posições $Y_{\alpha}^{\beta}$ segundo as expressões [2.45] e [2.46]. Portanto, a equação [2.47] escreve-se como:

$$
F_{\alpha}^{\beta(i n t)}=\left(\sum_{e f=1}^{n f} \int_{V o} \frac{\partial u_{e}}{\partial E_{k m}} \frac{\partial E_{k m}}{\partial Y_{\alpha}^{\beta}} d V o\right)=\left(\sum_{e f=1}^{n f} \int_{V o} S_{k m} \frac{\partial E_{k m}}{\partial Y_{\alpha}^{\beta}} d V o\right)
$$


$\mathrm{Na}$ qual, infere-se que a tensão de Piola-Kirchhoff de segunda espécie $S$ é o conjugado energético da medida de deformação de Green $E$. Explicitando o tensor de tensão para o estado plano de deformações chega-se a:

$$
\begin{gathered}
S_{11}=\frac{\partial u_{e}}{\partial E_{11}}=K\left\{(1-v) E_{11}+v E_{22}\right\} \\
S_{22}=\frac{\partial u_{e}}{\partial E_{22}}=K\left\{(1-v) E_{22}+v E_{11}\right\} \\
S_{12}=\frac{\partial u_{e}}{\partial E_{12}}=2 \mathbb{G} E_{12} \\
S_{21}=\frac{\partial u_{e}}{\partial E_{21}}=2 \mathbb{G} E_{21}
\end{gathered}
$$

Desenvolvendo a derivada do tensor de deformações de Green em relação aos parâmetros nodais encontra-se:

$$
\frac{\partial E}{\partial \vec{Y}}=\frac{1}{2} \frac{\partial C}{\partial \vec{Y}}=\frac{1}{2}\left[\left(A^{o}\right)^{-t} \cdot\left(\frac{\partial A^{1}}{\partial \vec{Y}}\right)^{t} \cdot A^{1} \cdot\left(A^{o}\right)^{-1}+\left(A^{o}\right)^{-t} \cdot\left(A^{1}\right)^{t} \cdot\left(\frac{\partial A^{1}}{\partial \vec{Y}}\right) \cdot\left(A^{o}\right)^{-1}\right]
$$

Onde o tensor $A^{1}$ é especificado pela equação [2.45] e suas derivadas em relação ao vetor posição atual $\vec{Y}$ (ou em notação indicial $Y_{\alpha}^{\beta}$ ) escrevem-se como:

$$
\frac{\partial A^{1}}{\partial Y_{1}^{\beta}}=\left[\begin{array}{cc}
\phi_{\beta, 1} & \phi_{\beta, 2} \\
0 & 0
\end{array}\right] \quad \frac{\partial A^{1}}{\partial Y_{2}^{\beta}}=\left[\begin{array}{cc}
0 & 0 \\
\phi_{\beta, 1} & \phi_{\beta, 2}
\end{array}\right]
$$

Nas expressões anteriores o índice sobrescrito $\beta$ define o nó e o índice subscrito refere-se à direção.

A partir das expressões [2.52] - [2.54] é possível calcular numericamente a integral descrita na equação [2.51] usando a quadratura de Hammer da seguinte forma:

$$
F_{\alpha}^{\beta(i n t)}=\left(\sum_{i h=1}^{n h} S_{k m} \frac{\partial E_{k m}}{\partial Y_{\alpha}^{\beta}} W_{i h} J\left(\left(\xi_{1}(i h), \xi_{2}(i h)\right)\right)\right)
$$

Sendo ih o contador que varia de 1 até o número de pontos de integração de Hammer $(i h), W_{i h}$ o peso correspondente ao ponto de integração $i h$ da técnica de integração numérica, $S_{k m}$ o segundo tensor de tensão de Piola-Kirchhoff definido pelas expressões [2.52], $\frac{\partial E_{k m}}{\partial Y_{\alpha}^{\beta}}$ a derivada do tensor de deformação de Green respeito 
às posições nodais determinado pelas equações [2.53] e [2.54] e $J\left(\left(\xi_{1}(i h), \xi_{2}(i h)\right)\right)$ o jacobiano da transformação avaliado no ponto de integração $i h$.

\subsubsection{Matriz hessiana do elemento tipo chapa}

Nesse item apresenta-se o cálculo da segunda derivada de energia de deformação em relação aos parâmetros nodais (Chamada de matriz hessiana estática na equação [2.19]) para elementos sólidos bidimensionais necessária para a aplicação do método de Newton-Raphson.

Retomando a expressão [2.51] para um elemento finito e aplicando a derivada respeito às posições nodais, obtém-se:

$$
H_{\alpha \beta z \theta}^{e s t a t i c a}=\frac{\partial^{2} U e}{\partial Y_{\alpha}^{\beta} \partial Y_{z}^{\theta}}=\frac{\partial F_{\alpha}^{\beta(\text { int })}}{\partial Y_{z}^{\theta}}=\frac{\partial}{\partial Y_{z}^{\theta}}\left(\int_{V o} S_{k m} \frac{\partial E_{k m}}{\partial Y_{\alpha}^{\beta}} d V o\right)
$$

Observa-se das equações [2.45], [2.52] e [2.53] que tanto o tensor de tensões $S_{k m}$ quanto o termo $\frac{\partial E_{k m}}{\partial \gamma_{\alpha}^{\beta}}$ são função das posições nodais. Dessa maneira, a derivada do vetor de forças internas é dada por:

$$
H_{\alpha \beta z \theta}^{e s t a t i c a}=\int_{V o}\left(S_{k m} \frac{\partial^{2} E_{k m}}{\partial Y_{\alpha}^{\beta} \partial Y_{z}^{\theta}}+\frac{\partial S_{k m}}{\partial Y_{z}^{\theta}} \frac{\partial E_{k m}}{\partial Y_{\alpha}^{\beta}}\right) d V o
$$

Na qual o termo $S_{k m} \mathrm{e} \frac{\partial E_{k m}}{\partial Y_{\alpha}^{\beta}}$ são determinados pelas expressões [2.52] e [2.53], respectivamente. A seguir mostra-se o cálculo dos termos restantes da equação [2.57].

Para facilitar a determinação da derivada do tensor de tensão em relação às posições, as equações contidas em [2.52] reescrevem-se da seguinte forma:

$$
\left\{\begin{array}{l}
S_{11} \\
S_{22} \\
S_{12} \\
S_{21}
\end{array}\right\}=\left[\begin{array}{cccc}
K(1-v) & K v & 0 & 0 \\
K v & K(1-v) & 0 & 0 \\
0 & 0 & 2 \mathbb{G} & 0 \\
0 & 0 & 0 & 2 \mathbb{G}
\end{array}\right] \cdot\left\{\begin{array}{l}
E_{11} \\
E_{22} \\
E_{12} \\
E_{21}
\end{array}\right\}
$$

Ás quais aplicam-se as derivadas obtendo: 


$$
\left\{\begin{array}{l}
\frac{\partial S_{11}}{\partial Y_{z}^{\theta}} \\
\frac{\partial S_{22}}{\partial Y_{z}^{\theta}} \\
\frac{\partial S_{12}}{\partial Y_{z}^{\theta}} \\
\frac{\partial S_{21}}{\partial Y_{z}^{\theta}}
\end{array}\right\}=\left[\begin{array}{cccc}
K(1-v) & K v & 0 & 0 \\
K v & K(1-v) & 0 & 0 \\
0 & 0 & 2 \mathbb{G} & 0 \\
0 & 0 & 0 & 2 \mathbb{G}
\end{array}\right] \cdot\left\{\begin{array}{c}
\frac{\partial E_{11}}{\partial Y_{z}^{\theta}} \\
\frac{\partial E_{22}}{\partial Y_{z}^{\theta}} \\
\frac{\partial E_{12}}{\partial Y_{z}^{\theta}} \\
\frac{\partial E_{21}}{\partial Y_{z}^{\theta}}
\end{array}\right\}
$$

Onde os termos $\frac{\partial E_{i j}}{\partial Y_{z}^{\theta}}$ foram calculados previamente segundo a equação [2.53]. Por conseguinte, somente resta definir a segunda derivada da deformação de Green em relação aos parâmetros nodais a qual pode ser expressa como:

$$
\frac{\partial^{2} E}{\partial \vec{Y} \partial \vec{Y}}=\frac{1}{2}\left[\left(A^{o}\right)^{-t} \cdot\left(\frac{\partial A^{1}}{\partial \vec{Y}}\right)^{t} \cdot\left(\frac{\partial A^{1}}{\partial \vec{Y}}\right) \cdot\left(A^{o}\right)^{-1}+\left(A^{o}\right)^{-t} \cdot\left(\frac{\partial A^{1}}{\partial \vec{Y}}\right)^{t} \cdot\left(\frac{\partial A^{1}}{\partial \vec{Y}}\right) \cdot\left(A^{o}\right)^{-1}\right]
$$

Já que observando as expressões [2.54] tem-se que:

$$
\frac{\partial^{2} A^{1}}{\partial \vec{Y} \partial \vec{Y}}=0
$$

Finalmente, de forma análoga como foi feito no cálculo das forças internas no item anterior é possível avaliar numericamente a integral expressa na equação [2.57] aplicando a quadratura de Hammer como segue:

$$
H_{\alpha \beta z \theta}^{\text {estatica }}=\sum_{i h=1}^{n h} h_{\alpha \beta z \theta} W_{i h} J\left(\left(\xi_{1}(i h), \xi_{2}(i h)\right)\right)
$$

Onde $h_{\alpha \beta z \theta}$ é a função a ser integrada definida por:

$$
h_{\alpha \beta \gamma z}=S_{k m} \frac{\partial^{2} E_{k m}}{\partial Y_{\alpha}^{\beta} \partial Y_{\gamma}^{z}}+\frac{\partial S_{k m}}{\partial Y_{\gamma}^{z}} \frac{\partial E_{k m}}{\partial Y_{\alpha}^{\beta}}
$$

Assim, depois de calcular a matriz hessiana estática pode ser determinada a matriz hessiana do sistema dinâmico segundo a expressão [2.19] e dessa forma continuar com o algoritmo de solução descrito nas equações [2.22] - [2.26]. 
2.2.3 Matriz de massa do elemento tipo chapa

Substituindo as aproximações propostas pela técnica dos elementos finitos na parcela da variação da energia cinética (Ver equação [2.4]), obtém-se:

$$
\int_{V o} \rho_{o} \phi_{l}\left(\xi_{1}, \xi_{2}\right) \phi_{k}\left(\xi_{1}, \xi_{2}\right) d V o \ddot{Y}_{i}^{l} \delta Y_{i}^{l}=M \cdot \ddot{\vec{Y}} \cdot \overrightarrow{\delta Y}
$$

Onde $\phi_{l}$ e $\phi_{k}$ referem-se às funções de forma associadas aos nós $l$ e $k$.Dessa forma, a matriz de massa é dada por:

$$
M_{l k}=\int_{V o} \rho_{o} \phi_{l}\left(\xi_{1}, \xi_{2}\right) \phi_{k}\left(\xi_{1}, \xi_{2}\right) d V o
$$

Nota-se que as grandezas envolvidas não são constantes no domínio de integração por tanto, emprega-se a quadratura de Hammer para sua avaliação como mostrado abaixo:

$$
M_{l k}=\rho_{0} b_{0} W_{i h} \phi_{l}\left(\xi_{1}(i h), \xi_{2}(i h)\right) \phi_{k}\left(\xi_{1}(i h), \xi_{2}(i h)\right) \operatorname{Det}\left[A^{0}\left(\xi_{1}(i h), \xi_{2}(i h)\right)\right]
$$

$\mathrm{Na}$ qual, $b_{0}$ é a largura do elemento finito na configuração inicial, $\phi_{l}\left(\xi_{1}(i h), \xi_{2}(i h)\right)$ e $\operatorname{Det}\left[A^{0}\left(\xi_{1}(i h), \xi_{2}(i h)\right)\right]$ representam a função de forma e o determinante da matriz $A^{0}$ avaliados no ponto de Hammer (ih), respectivamente, e $W_{i h}$ denota o peso utilizado na técnica de integração numérica.

\subsubsection{Exemplo 1 - Viga engastada submetida a um carregamento transversal variável no tempo}

Nesse exemplo analisa-se a resposta dinâmica de uma viga engastada (ver figura 12), sem amortecimento, submetida a um carregamento transversal variável no tempo (ver figura 13). Esse exemplo encontra-se nos trabalhos de Behdinan et al. (1998) e Greco (2004). Nesse exemplo foi adotado o mesmo sistema de unidade adotado pelas referencias. 


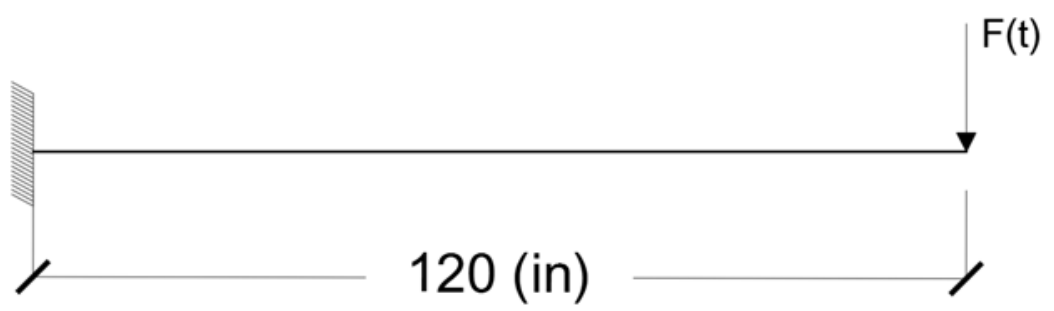

Figura 12 - Viga engastada submetida a um carregamento transversal variável no tempo.

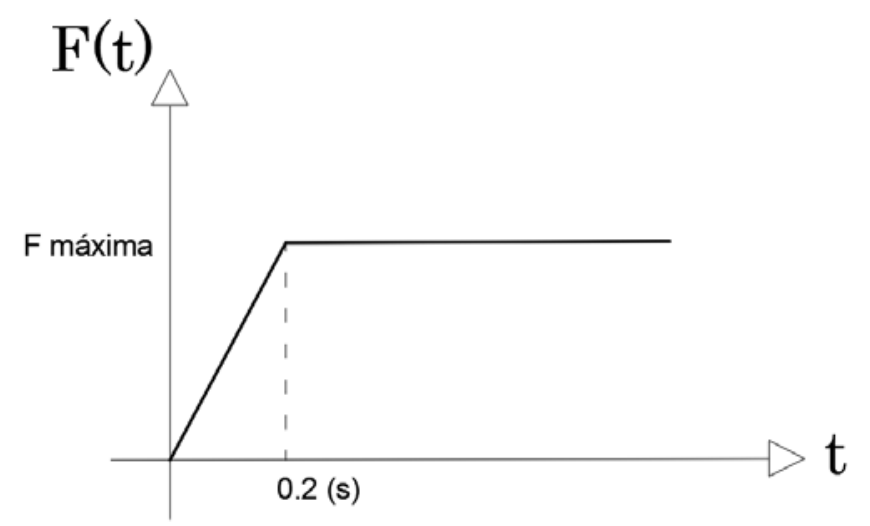

Figura 13 - Força transversal aplicada no extremo livre da viga versus tempo.

O resumo dos dados de entrada para o problema apresentam-se na tabela 2.

\begin{tabular}{|lr|}
\hline \multicolumn{2}{|c|}{ Dados de entrada } \\
$b$ [in] & 1 \\
$h$ [in] & 10,627 \\
$L$ [in] & 120 \\
$\rho\left[\right.$ [lb-s $\left.{ }^{2} / i^{4}\right]$ & 0,0094116 \\
E [Psi] & 30000000 \\
$G$ [Psi] & 15000000 \\
$\Delta t[s]$ & 0,01 \\
\hline
\end{tabular}

Tabela 2 - Dados de entrada para análise de viga engastada submetida a um carregamento variável no tempo.

A seguir, apresentam-se os resultados de deslocamento vertical e horizontal do extremo livre da viga para uma força aplicada máxima de 1E+05 libras e 5E+05 libras. $\mathrm{Na}$ discretização empregaram-se 98 elementos finitos triangulares 
isoparamétricos de aproximação cubica. No que diz respeito aos parâmetros do algoritmo de integração temporal, adotaram-se os valores correspondentes ao método de Newmark para aceleração media constante.

Nas figuras 16 e 17 apresenta-se a configuração deslocada da viga para o caso de carga $F=1 E+05$ libras nos instantes de tempo $t=0.25$ segundos e $t=0.35$ segundos. Ditas imagens foram geradas no software de pós-processamento Acadview (PACCOLA; CODA, 2005).

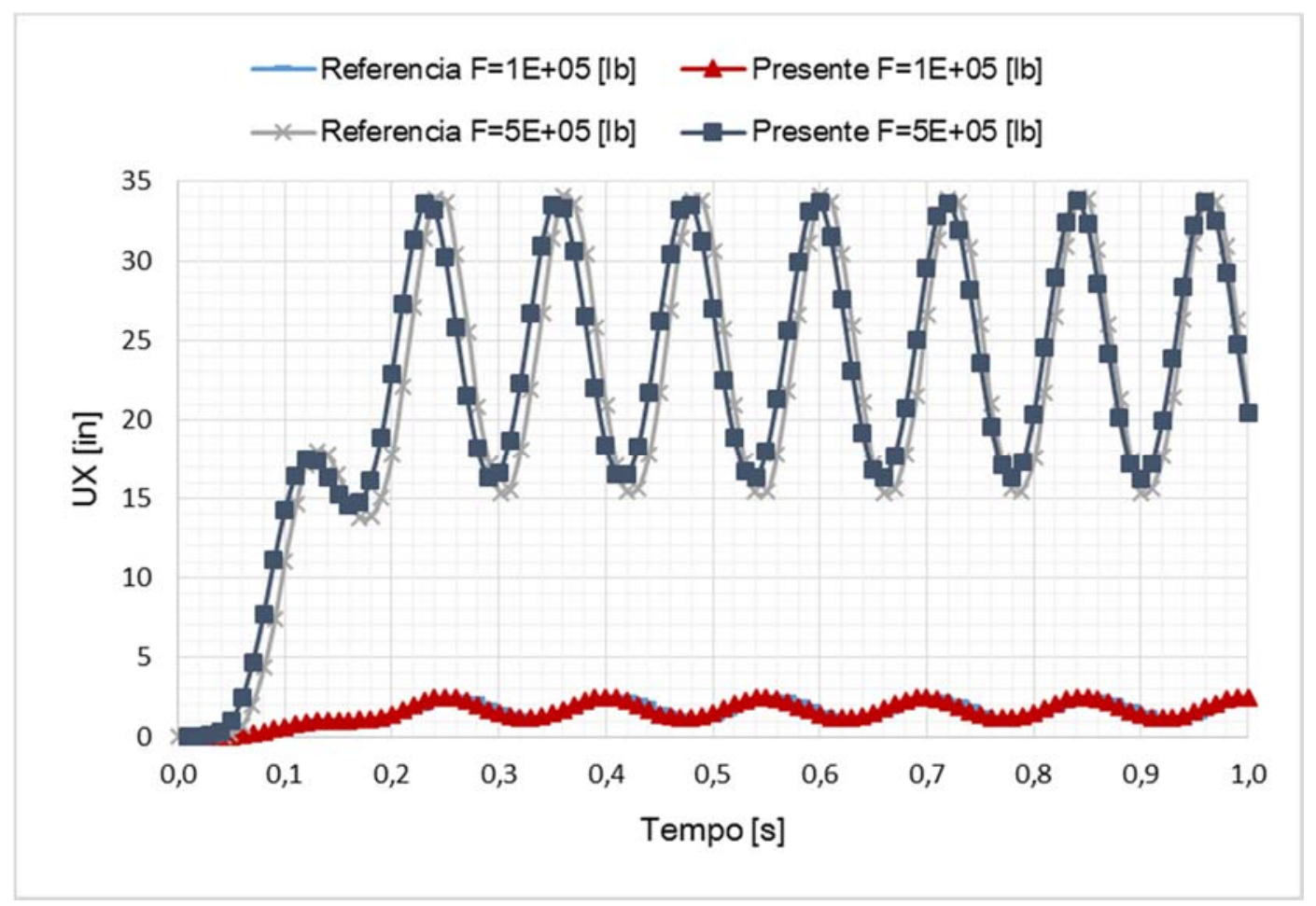

Figura 14 - Deslocamento horizontal para valores de força aplicada máxima de F=1E+05 e $\mathrm{F}=5 \mathrm{E}+05$ libras. 


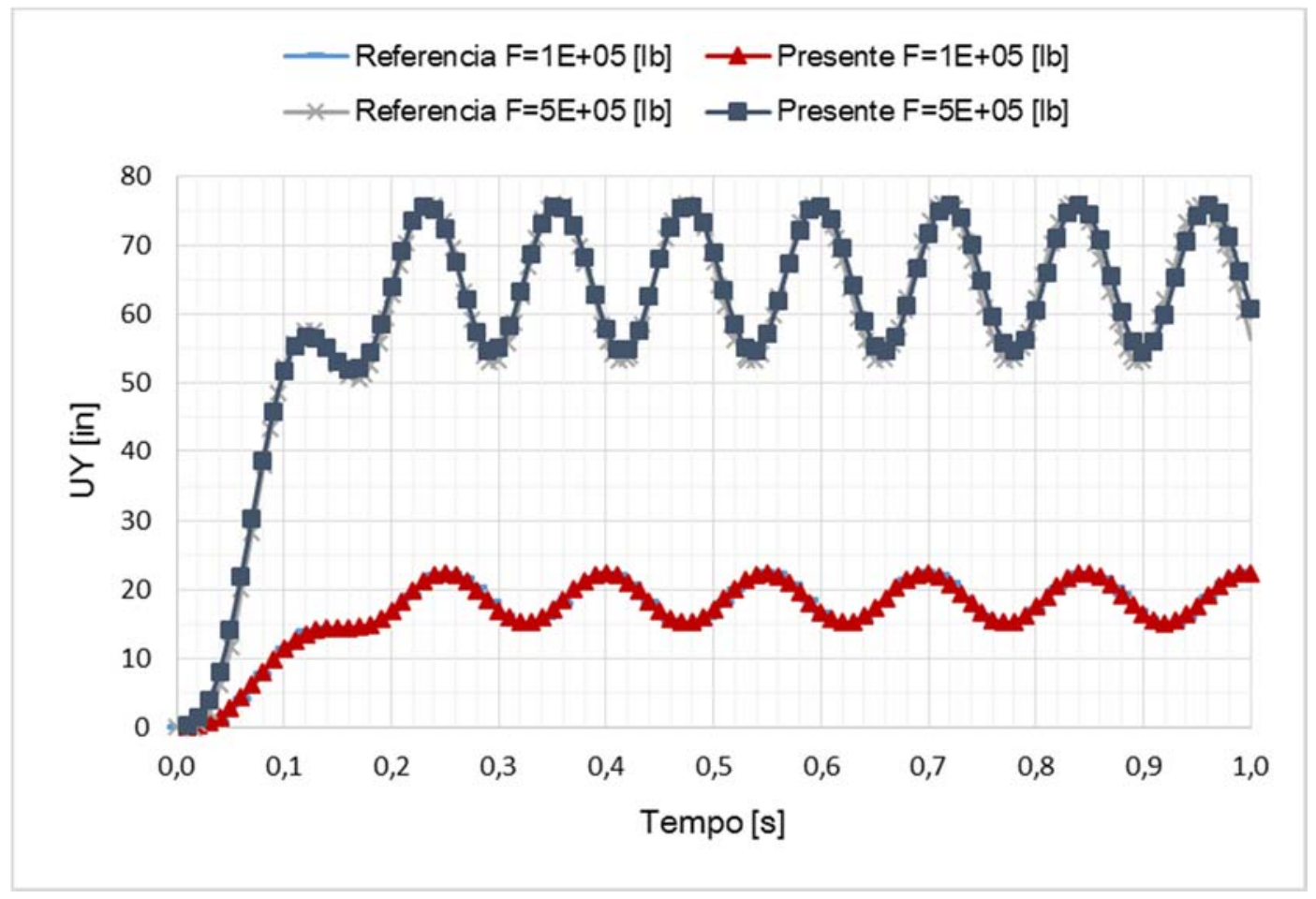

Figura 15 - Deslocamento vertical para valores de força aplicada máxima de F=1E+05 e $F=5 E+05$ libras.

\section{Deslocamento horizontal}

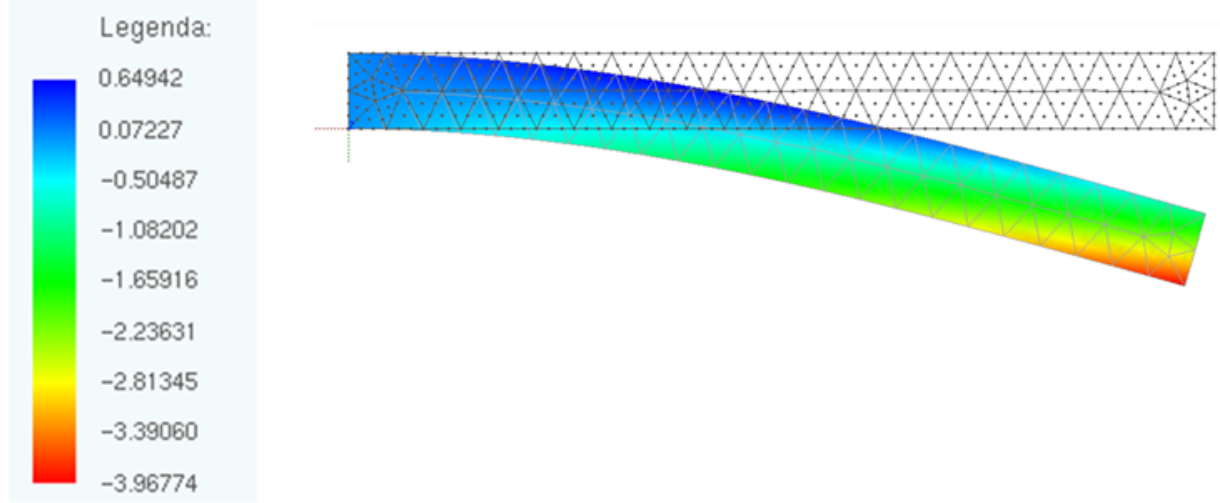

Deslocamento vertical

Legenda:

0.00000

$-2.82111$

$-5.64222$

$-8.46334$

$-11.28445$

$-14.10556$

$-16.92667$

$-19.74779$

$-22.56890$

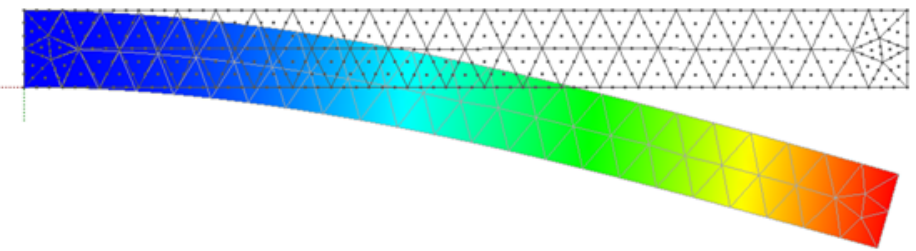

Figura 16 - Configuração deslocada (in) para $t=0.25$ segundos. 


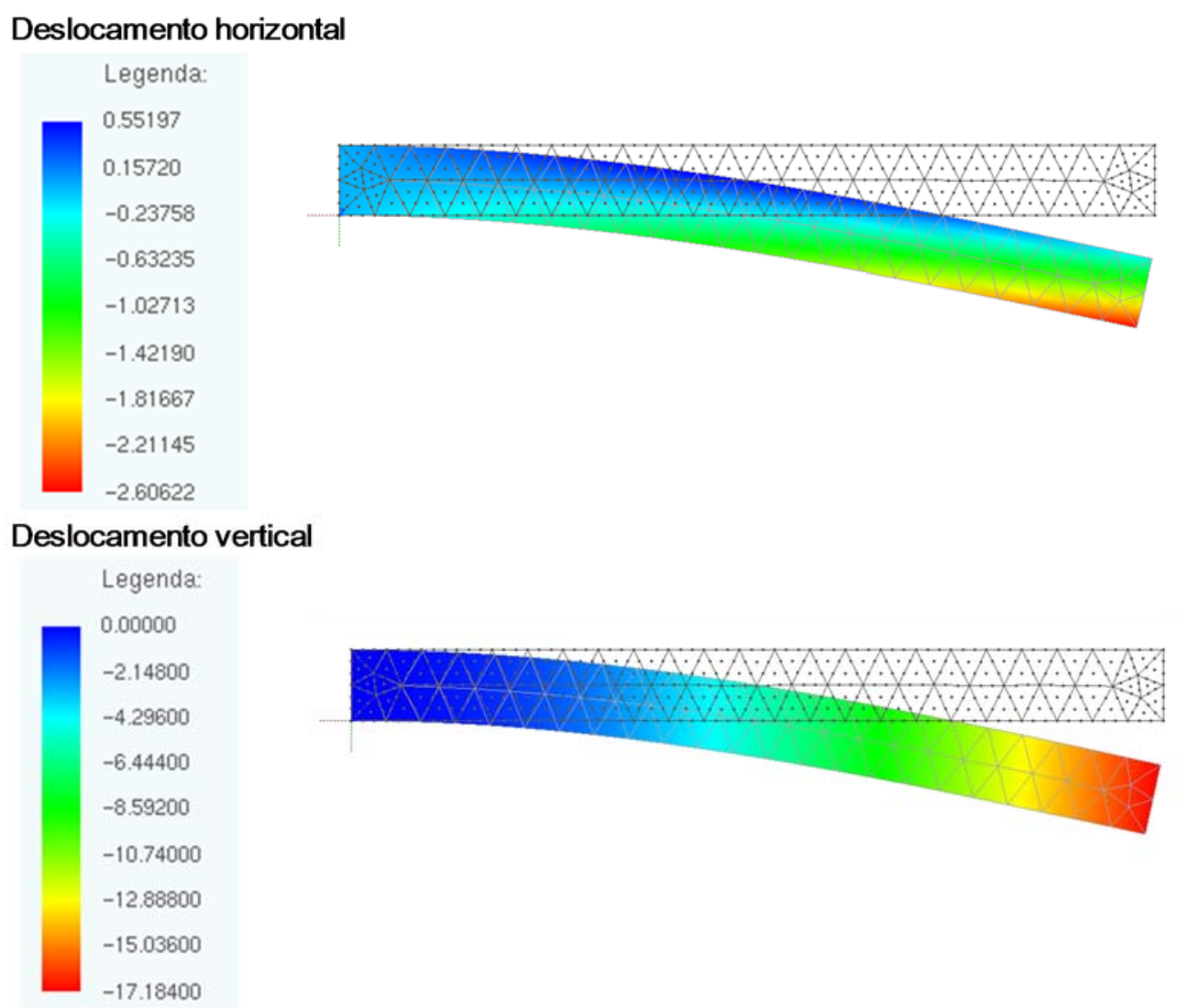

Figura 17 - Configuração deslocada (in) para $\mathrm{t}=0.35$ segundos.

2.2.5 Exemplo 2 - Viga engastada com amortecimento submetida a um carregamento transversal variável no tempo

Com o objetivo de testar a formulação relacionada à parcela de amortecimento, analisa-se a mesma estrutura do exemplo anterior submetida a um carregamento definido pela função triangular mostrada abaixo e com um coeficiente de amortecimento viscoso de $0.1 \frac{l b-s^{2}}{\text { in }}$ em cada grau de liberdade (Ver tabela 3). 


\begin{tabular}{|lr|}
\hline \multicolumn{2}{|c|}{ Dados de entrada } \\
$b$ [in $]$ & 1 \\
$h$ [in $]$ & 10,627 \\
$\mathrm{~L}[\mathrm{in}]$ & 120 \\
$\rho\left[\mathrm{lb}-\mathrm{s}^{2} / \mathrm{in}^{4}\right]$ & 0,0094116 \\
$\mathrm{C}[\mathrm{lb} / \mathrm{in} / \mathrm{s}]$ & 0,1 \\
$\mathrm{E}[\mathrm{Psi}]$ & 30000000 \\
$\mathrm{G}[\mathrm{Psi}]$ & 15000000 \\
$\Delta \mathrm{t}[\mathrm{s}]$ & 0,01 \\
\hline
\end{tabular}

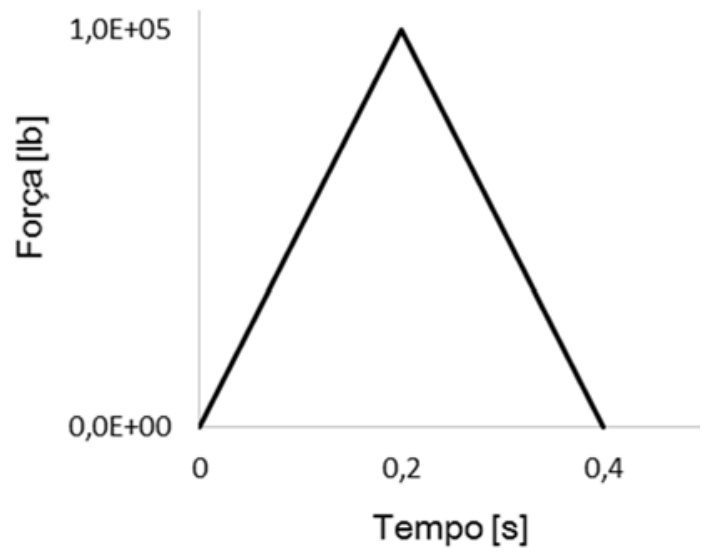

Tabela 3 - Dados de entrada para análise de viga engasta submetida a um carregamento variável no tempo e considerando amortecimento.

Esse exemplo encontra-se no trabalho de Behdinan, Stylianou, e Tabarrok (1998) onde foi empregada a formulação co-rotacional a elementos tipo barra com cinemática de Euler-Bernoulli.

Na figura 18 encontra-se a solução numérica, do trabalho de referência e do presente trabalho, para o deslocamento vertical do extremo livre da viga.

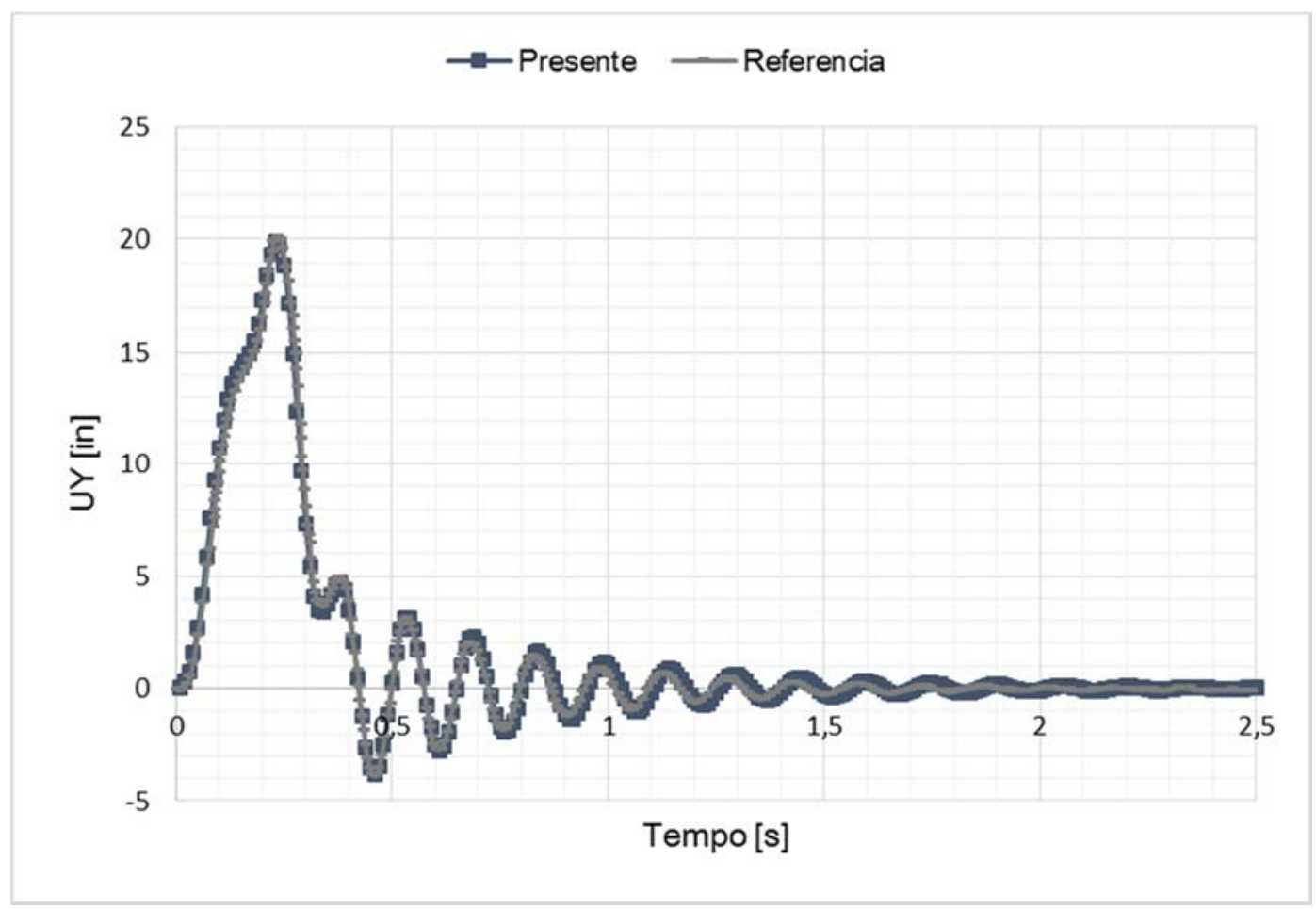

Figura 18 - Deslocamento vertical do extremo livre da viga. 


\subsection{Elemento de pórtico}

A diferença entre o elemento de pórtico e o elemento de chapa é a imposição da cinemática de Reissner-Timoshenko no comportamento da seção transversal, inexistente em elemento de chapa (ou sólido bidimensional). A formulação apresentada nesse item é descrita detalhadamente no trabalho de Coda e Paccola (2014) e Siqueira (2016).

Como é bem sabido, a cinemática de Reissner-Timoshenko leva em cosideração o efeito das tensões cisalhantes o qual implica que a seção transversal apesar de permanecer plana após a deformação do elemento, ésta não forma um ângulo ortogonal à linha de referência.

Na figura 19, mostrada abaixo, mostra-se esquematicamente a configuração inicial e final de um elemento pórtico e o espaço adimensional auxiliar a ser empregado de forma análoga como foi feito no caso do elemento chapa. Esse espaço adimensional é definido pelas coordenadas $\xi_{1}$ e $\xi_{2}$, com a característica que a coordenada $\xi_{1}$ coincide com a linha de referência (eixo) da barra na configuração inicial e final bem como a coordenada $\xi_{2}$ alinha-se com o plano da seção transversal.

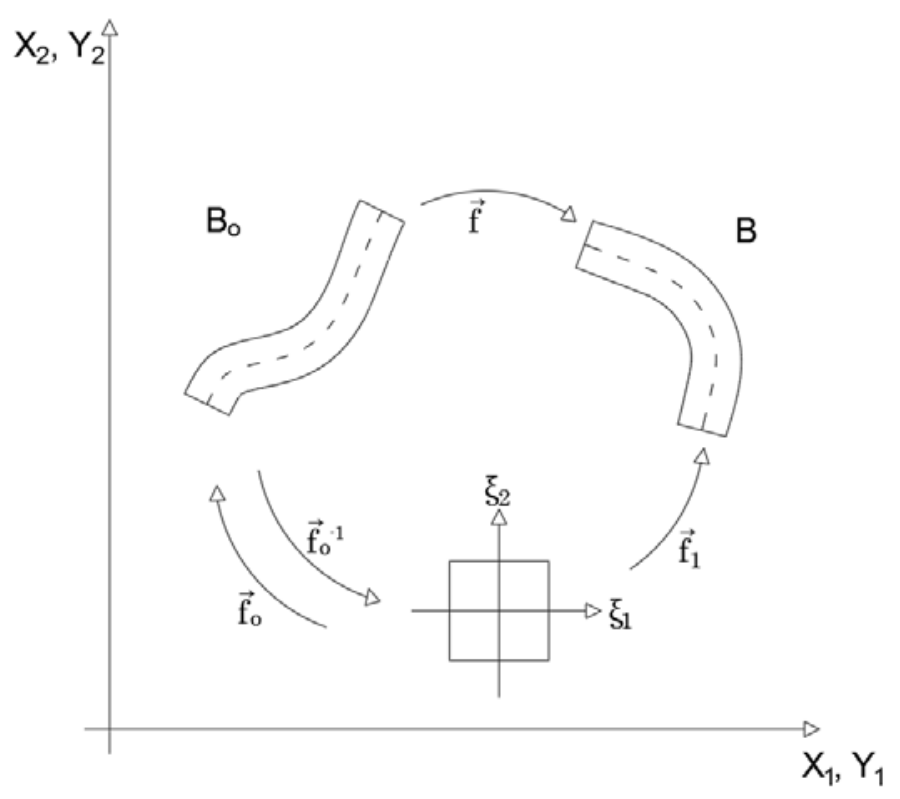

Figura 19. Mapeamento das configurações e a sua relação com o espaço adimensional. 
Partindo da linha de referência, o mapeamento da configuração inicial pode ser escrito da seguinte maneira:

$$
f_{0 i}^{m}\left(\xi_{1}\right)=X_{i}^{m}\left(\xi_{1}\right)=\phi_{l}\left(\xi_{1}\right) X_{i l}^{m}
$$

Onde os índices $m$ e 0 referem-se à linha de referência da barra e à configuração inicial do corpo, respectivamente. Dessa forma, entende-se $\overrightarrow{f_{0}^{m}}\left(\xi_{1}\right)$ como a função de mapeamento da linha de referência na configuração inicial do corpo e $X_{i}^{m}\left(\xi_{1}\right)$ como a posição inicial da linha de referência na direção $i$ a qual é aproximada pelo método dos elementos finitos como $\phi_{l}\left(\xi_{1}\right) X_{i l}^{m}$ sendo $\phi_{l}$ a função de forma associada ao nó $l$ e o $X_{i l}^{m}$ o valor nodal da posição inicial.

Posto que na formulação do elemento pórtico o ângulo da seção transversal também é considerado um parâmetro nodal e devido à cinemática adotada, ele é aproximado de forma análoga à posição como:

$$
\theta_{k}^{0}\left(\xi_{1}\right)=\phi_{l}\left(\xi_{1}\right) \theta_{l}^{0}
$$

Na qual os ângulos nodais $\theta_{l}^{0}$ a serem introduzidos na equação anterior são determinados por:

$$
\theta_{k}^{0}=\tan ^{-1}\left(v_{2(k)} / v_{1(k)}\right)
$$

É importante destacar que em concordância com a notação indicial os índices que se encontram dentro de parênteses não representam soma. Na equação anterior $v_{1(k)}$ e $v_{2(k)}$ representam as componentes do vetor unitário normal à linha de referência, calculados como:

$$
v_{1(k)}=-T_{2 k} /\|\vec{T}\| \quad v_{2(k)}=T_{1 k} /\|\vec{T}\|
$$

Onde $\vec{T}$ é o vetor tangente à linha de referência e portanto $\|\vec{T}\|, T_{1 k}$ e $T_{2 k}$ são a sua norma euclidiana e a suas componentes na direção 1 e 2, respectivamente. Esse vetor é aproximado segundo a seguinte expressão: 


$$
T_{i k}=\left.\frac{d \phi_{l}\left(\xi_{1}\right)}{d \xi_{1}}\right|_{\xi_{1}^{k}} X_{i l}^{m}
$$

Depois de ter definido as aproximações do continuo pelo método dos elementos finitos posicional, é possível escrever a posição de um ponto qualquer contido no domínio da configuração inicial como:

$$
\begin{aligned}
& f_{01}\left(\xi_{1}, \xi_{2}\right)=X_{1}\left(\xi_{1}, \xi_{2}\right)=\phi_{l}\left(\xi_{1}\right) X_{1}^{l}+\frac{h}{2} \xi_{2} \cos \left(\phi_{l}\left(\xi_{1}\right) \theta_{l}^{0}\right) \\
& f_{02}\left(\xi_{1}, \xi_{2}\right)=X_{2}\left(\xi_{1}, \xi_{2}\right)=\phi_{l}\left(\xi_{1}\right) X_{2}^{l}+\frac{h}{2} \xi_{2} \sin \left(\phi_{l}\left(\xi_{1}\right) \theta_{l}^{0}\right)
\end{aligned}
$$

Onde $h$ denota a altura da barra na configuração inicial.

De forma similar constrói-se a função de mapeamento do corpo na configuração atual $\overrightarrow{f_{1}}$, ficando da seguinte forma:

$$
\begin{aligned}
& f_{11}\left(\xi_{1}, \xi_{2}\right)=Y_{1}\left(\xi_{1}, \xi_{2}\right)=\phi_{l}\left(\xi_{1}\right) Y_{1}^{l}+\frac{h}{2} \xi_{2} \cos \left(\phi_{l}\left(\xi_{1}\right) \theta_{l}\right) \\
& f_{12}\left(\xi_{1}, \xi_{2}\right)=Y_{2}\left(\xi_{1}, \xi_{2}\right)=\phi_{l}\left(\xi_{1}\right) Y_{2}^{l}+\frac{h}{2} \xi_{2} \sin \left(\phi_{l}\left(\xi_{1}\right) \theta_{l}\right)
\end{aligned}
$$

Onde $Y_{i}^{l}$ designa a posição atual na direção $i$ do nó $l$ e $\theta_{l}$ é o ângulo da seção transversal no nó $l$.

Deve-se lembrar que a posição atual obtém-se a partir de valores tentativos que são corrigidos iterativamente, de modo que é possível calcular numericamente as equações [2.73]. Além disso, nota-se que a altura inicial da barra é também empregada no mapeamento da configuração atual, portanto, para evitar travamento volumétrico adota-se a lei constitutiva com valor nulo para o coeficiente de Poisson como será mostrado posteriormente.

Tendo definidas as funções de mapeamento inicial $\overrightarrow{f_{0}}$ e atual $\overrightarrow{f_{1}}$ pode-se escrever a função mudança de configuração $\vec{f}$ como (Ver figura 19):

$$
\vec{f}=\overrightarrow{f_{1}} \cdot\left(\overrightarrow{f_{0}}\right)^{-1}
$$

E o gradiente de $\vec{f}$ como:

$$
A=A^{1} \cdot\left(A^{0}\right)^{-1}
$$


Onde $A^{0}$ e $A^{1}$ são os gradientes das funções $\overrightarrow{f_{0}}$ e $\overrightarrow{f_{1}}$ definidos por as seguintes expressões, respectivamente:

$$
\begin{gathered}
A_{i j}^{0}=\frac{d f_{0 i}}{d \xi_{j}}=f_{0 i, j}=\left[\begin{array}{ll}
\phi_{l, 1} X_{1 l}-\frac{h}{2} \xi_{2} \sin \left(\phi_{k} \theta_{k}^{0}\right)\left(\phi_{z, 1} \theta_{z}^{0}\right) & \frac{h}{2} \cos \left(\phi_{l} \theta_{l}^{0}\right) \\
\phi_{l, 1} X_{2 l}+\frac{h}{2} \xi_{2} \cos \left(\phi_{k} \theta_{k}^{0}\right)\left(\phi_{z, 1} \theta_{z}^{0}\right) & \frac{h}{2} \sin \left(\phi_{l} \theta_{l}^{0}\right)
\end{array}\right] \\
A_{i j}^{1}=\frac{d f_{1 i}}{d \xi_{j}}=f_{1 i, j}=\left[\begin{array}{ll}
\phi_{l, 1} Y_{1 l}-\frac{h}{2} \xi_{2} \sin \left(\phi_{k} \theta_{k}\right)\left(\phi_{z, 1} \theta_{z}\right) & \frac{h}{2} \cos \left(\phi_{l} \theta_{l}\right) \\
\phi_{l, 1} Y_{2 l}+\frac{h}{2} \xi_{2} \cos \left(\phi_{k} \theta_{k}\right)\left(\phi_{z, 1} \theta_{z}\right) & \frac{h}{2} \sin \left(\phi_{l} \theta_{l}\right)
\end{array}\right]
\end{gathered}
$$

$\mathrm{Na}$ qual $\phi_{l, \mathrm{i}}$ indica a derivada da função de forma associada ao nó $l$ respeito à variável adimensional na direção $i$. Finalmente, pode-se calcular o tensor de deformação de Green-Lagrange como:

$$
E=\frac{1}{2}(\mathcal{C}-I)=\frac{1}{2}\left(A^{t} \cdot A-I\right)
$$

\subsubsection{Forças internas do elemento tipo pórtico}

Como foi feito no cálculo das forças internas de elementos chapa, aproveitase as definições de força e tensão de segunda espécie de Piola-Kirchhoff como conjugados energéticos da posição e da deformação de Green, respectivamente, para expressar a variação da energia de deformação como segue:

$$
\begin{gathered}
\delta U e=\frac{\partial U e}{\partial \vec{Y}} \cdot \delta \vec{Y}=\overrightarrow{F^{\imath n t}} \cdot \delta \vec{Y}=\int_{V o} \frac{\partial u_{e}}{\partial E}: \frac{\partial E}{\partial \vec{Y}} d V o \cdot \delta \vec{Y} \\
=\int_{V o} S: \frac{\partial E}{\partial \vec{Y}} d V o \cdot \delta \vec{Y}
\end{gathered}
$$

Onde o tensor de tensões $S$ é dado por:

$$
S=\frac{\partial u_{e}}{\partial E}=\left[\begin{array}{cc}
\mathbb{E} E_{11} & 2 \mathbb{G} E_{12} \\
2 \mathbb{G} E_{12} & \mathbb{E} E_{22}
\end{array}\right]
$$

Lembrando que $\mathbb{E}$ pode ser entendido como o modulo de Young para o caso de pequenas deformações e $\mathbb{G}$ como o modulo de elasticidade transversal. Apesar do cálculo da derivada da deformação de Green em relação aos parâmetros nodais 
ser dado pela mesma expressão apresentada no item referente ao elemento solido bidimensional [2.53], por conveniência, é apresentada de novo nesse item:

$$
\begin{gathered}
\frac{\partial E}{\partial \vec{Y}}=\frac{\partial}{\partial \vec{Y}}\left[\frac{1}{2}\left(A^{t} \cdot A-I\right)\right] \\
\frac{\partial E}{\partial \vec{Y}}=\frac{1}{2}\left\{\left(A^{o}\right)^{-t} \cdot\left(\frac{\partial A^{1}}{\partial \vec{Y}}\right)^{t} \cdot A^{1} \cdot\left(A^{o}\right)^{-1}+\left(A^{o}\right)^{-t} \cdot\left(A^{1}\right)^{t} \cdot\left(\frac{\partial A^{1}}{\partial \vec{Y}}\right) \cdot\left(A^{o}\right)^{-1}\right\}
\end{gathered}
$$

É importante observar que o tensor gradiente $A^{1}$ é função dos parâmetros nodais que representam a posição nas duas direções do plano e do ângulo de inclinação da seção transversal, portanto a derivada do $A^{1}$ em relação aos parâmetros nodais mencionados expressa-se como:

$$
\begin{aligned}
& \frac{\partial A^{1}}{\partial Y_{1}^{\beta}}=\left[\begin{array}{cc}
\phi_{\beta, 1} & 0 \\
0 & 0
\end{array}\right] \quad \frac{\partial A^{1}}{\partial Y_{2}^{\beta}}=\left[\begin{array}{cc}
0 & 0 \\
\phi_{\beta, 1} & 0
\end{array}\right] \\
& \frac{\partial A^{1}}{\partial Y_{3}^{\beta}}=\left[\begin{array}{cc}
-\frac{h}{2} \xi_{2}\left[\cos \left(\phi_{k} \theta_{k}\right)\left(\phi_{z, 1} \theta_{z}\right) \phi_{\beta}+\sin \left(\phi_{k} \theta_{k}\right) \phi_{\beta, 1}\right] & -\frac{h}{2} \sin \left(\phi_{l} \theta_{l}\right) \phi_{\beta} \\
\frac{h}{2} \xi_{2}\left[\cos \left(\phi_{k} \theta_{k}\right) \phi_{\beta, 1}-\sin \left(\phi_{k} \theta_{k}\right)\left(\phi_{z, 1} \theta_{z}\right) \phi_{\beta, 1}\right] & \frac{h}{2} \cos \left(\phi_{l} \theta_{l}\right) \phi_{\beta}
\end{array}\right]
\end{aligned}
$$

Onde o índice subscrito de $Y$ indica a direção quando tomar valor de 1 ou 2 e refere-se ao ângulo da seção transversal quando for 3. Dessa forma, tem sido definidos todos os termos necessários para o cálculo da integral da equação [2.79] dando como resultado o vetor de forças internas. Devido à geometria do domínio de integração emprega-se a quadratura de Gauss como técnica de integração numérica:

$$
\vec{F} i n t=b_{0} w_{i g} w_{j g} S\left(\xi_{1 i g}, \xi_{2 j g}\right): \frac{\partial E}{\partial \vec{Y}}\left(\xi_{1 i g}, \xi_{2 j g}\right) \operatorname{Det}\left[A^{0}\left(\xi_{1 i g}, \xi_{2 j g}\right)\right]
$$

Na qual $w_{i g}$ e $w_{j g}$ são os pesos de Gauss para os pontos de integração $\xi_{1 i g}$ e $\xi_{2 j g}$, respectivamente, e os índices ig e jg percorrem os pontos de Gauss estabelecidos para a direção 1 e 2 , respectivamente. 


\subsubsection{Matriz hessiana do elemento tipo pórtico}

Segundo a equação [2.19] a matriz hessiana do sistema dinâmico é o gradiente do vetor $\vec{g}$. Para analises estáticas basta desconsiderar as parcelas decorrentes da energia cinética e de amortecimento.

A seguir mostra-se o cálculo da primeira parcela da equação [2.19] correspondente à segunda derivada da energia de deformação de elementos pórtico em relação aos parâmetros nodais, chamada de matriz hessiana estática:

$$
\frac{\partial^{2} U e}{\partial \vec{Y} \partial \vec{Y}}=\int_{V o} \frac{\partial^{2} u_{e}}{\partial \vec{Y} \partial \vec{Y}} d V o=\int_{V o} \frac{\partial \overrightarrow{F^{i n t}}}{\partial \vec{Y}} d V o=\int_{V o}\left(\frac{\partial S}{\partial \vec{Y}}: \frac{\partial E}{\partial \vec{Y}}+S: \frac{\partial^{2} E}{\partial \vec{Y} \partial \vec{Y}}\right) d V o
$$

Onde os termos $\frac{\partial E}{\partial \vec{Y}}$ e $S$ são conhecidos já que foram calculados para a montagem do vetor de forças internas. Portanto, somente falta resolver as seguintes derivadas: $\frac{\partial S}{\partial \vec{Y}} \mathrm{e} \frac{\partial^{2} E}{\partial \vec{Y} \partial \vec{Y}}$. A primeira delas, a derivada do tensor de tensões de segunda espécie de Piola-Kirchhoff em relação às posições atuais é dada por:

$$
\frac{\partial S}{\partial \vec{Y}}=\frac{\partial}{\partial \vec{Y}}\left[\begin{array}{cc}
\mathbb{E} E_{11} & 2 \mathbb{G} E_{12} \\
2 \mathbb{G} E_{12} & \mathbb{E} E_{22}
\end{array}\right]=\left[\begin{array}{cc}
\mathbb{E} \frac{\partial E_{11}}{\partial \vec{Y}} & 2 \mathbb{G} \frac{\partial E_{12}}{\partial \vec{Y}} \\
2 \mathbb{G} \frac{\partial E_{21}}{\partial \vec{Y}} & \mathbb{E} \frac{\partial E_{22}}{\partial \vec{Y}}
\end{array}\right]
$$

Na qual, mais uma vez precisa-se da derivada da deformação de Green que já é conhecida. Assim, o seguinte passo é determinar a segunda derivada da deformação de Green em relação ao vetor $\vec{Y}$ como segue:

$$
\frac{\partial^{2} E}{\partial \vec{Y} \partial \vec{Y}}=\frac{1}{2}\left\{2\left(A^{o}\right)^{-t} \cdot\left(\frac{\partial A^{1}}{\partial \vec{Y}}\right)^{t} \cdot\left(\frac{\partial A^{1}}{\partial \vec{Y}}\right) \cdot\left(A^{o}\right)^{-1}+O+O^{t}\right\}
$$

Onde:

$$
O=\left(A^{o}\right)^{-t} \cdot\left(A^{1}\right)^{t} \cdot \frac{\partial^{2} A^{1}}{\partial \vec{Y} \partial \vec{Y}} \cdot\left(A^{0}\right)^{-1}
$$

Nestas expressões o único termo que falta definir é $\frac{\partial^{2} A^{1}}{\partial \vec{Y} \partial \bar{Y}}$, entretanto, este assume valores não nulos somente para os graus de liberdade associados ao giro da seção transversal: 


$$
\begin{aligned}
& \frac{\partial^{2} A^{1}}{\partial Y_{3}^{\beta} \partial Y_{3}^{\mu}} \\
& =\left[\begin{array}{cc}
-\frac{h}{2} \xi_{2}\left[\begin{array}{c}
\cos \left(\phi_{k} \theta_{k}\right)\left(\phi_{\mu, 1} \phi_{\beta}\right)+ \\
\cos \left(\phi_{k} \theta_{k}\right)\left(\phi_{\beta, 1} \phi_{\mu}\right)-\sin \left(\phi_{k} \theta_{k}\right)\left(\phi_{z, 1} \theta_{z}\right) \phi_{\beta} \phi_{\mu}
\end{array}\right] & -\frac{h}{2} \cos \left(\phi_{l} \theta_{l}\right) \phi_{\beta} \phi_{\mu} \\
-\frac{h}{2} \xi_{2}\left[\begin{array}{c}
\sin \left(\phi_{k} \theta_{k}\right)\left(\phi_{\beta, 1} \phi_{\mu}\right)+ \\
\sin \left(\phi_{k} \theta_{k}\right)\left(\phi_{\mu, 1} \phi_{\beta}\right)+\cos \left(\phi_{k} \theta_{k}\right)\left(\phi_{z, 1} \theta_{z}\right) \phi_{\beta} \phi_{m}
\end{array}\right] & -\frac{h}{2} \sin \left(\phi_{l} \theta_{l}\right) \phi_{\beta} \phi_{\mu}
\end{array}\right]
\end{aligned}
$$

Sendo $\beta$ e $\mu$ os nós do elemento. Finalmente a integração numérica da matriz hessiana é feita aplicando a quadratura de Gauss Legendre, de maneira análoga ao cálculo das forças internas.

$$
\begin{aligned}
\frac{\partial^{2} U e}{\partial \vec{Y} \partial \vec{Y}}=b_{0} w_{i g} w_{j g}\left\{\frac{\partial S}{\partial \vec{Y}}\left(\xi_{1 i g}, \xi_{2 j g}\right): \frac{\partial E}{\partial \vec{Y}}\left(\xi_{1 i g}, \xi_{2 j g}\right)\right. \\
\left.+S\left(\xi_{1 i g}, \xi_{2 j g}\right): \frac{\partial^{2} E}{\partial \vec{Y} \partial \vec{Y}}\left(\xi_{1 i g}, \xi_{2 j g}\right)\right\} \operatorname{Det}\left[A^{0}\left(\xi_{1 i g}, \xi_{2 j g}\right)\right]
\end{aligned}
$$

Na qual $w_{i g}$ e $w_{i g}$ são os pesos de Gauss para os pontos de integração $\xi_{1 i g}$ e $\xi_{2 j g}$, respectivamente.

\subsubsection{Matriz de massa do elemento tipo pórtico}

As aproximações da técnica dos elementos finitos para a posição e aceleração a serem introduzidas na variação da energia cinética (Equação [2.4]) são dadas respectivamente por:

$$
\begin{aligned}
\vec{y}^{m} & =\vec{\phi} \cdot \vec{Y} \\
\ddot{\vec{y}}^{m} & =\vec{\phi} \cdot \ddot{\vec{Y}}
\end{aligned}
$$

Na qual o índice sobrescrito " $m$ " refere-se à linha de referência do elemento finito de pórtico. Assim sendo, a equação [2.4] reescreve-se como:

$$
\int_{V o} \rho_{o} \phi_{l}\left(\xi_{1}, \xi_{2}\right) \phi_{k}\left(\xi_{1}, \xi_{2}\right) d V o \ddot{Y}_{i}^{l} \delta Y_{i}^{l}=M \cdot \ddot{\vec{Y}} \cdot \overrightarrow{\delta Y}
$$

A qual será integrada empregando a quadratura de Gauss da mesma forma como foi avaliado o vetor de forças internas e a matriz hessiana nos itens anteriores, resultado na seguinte expressão: 
$M_{l k}=\rho_{0} b_{0} w_{i g} w_{j g} \phi_{l}\left(\xi_{1}(i h), \xi_{2}(i h)\right) \phi_{k}\left(\xi_{1}(i h), \xi_{2}(i h)\right) \operatorname{Det}\left[A^{0}\left(\xi_{1}(i h), \xi_{2}(i h)\right)\right]$

É importante ressaltar que devido às simplificações contidas nas expressões [2.90] para a posição e a aceleração, a inercia rotacional da barra é desprezada portanto os termos da matriz de massa associados ao ângulo da seção transversal são nulos, consequentemente, esses termos na matriz de amortecimento também o serão já que o modelo de amortecimento adotado é o modelo de Rayleigh (Proporcional à matriz de massa. $\alpha_{2}=0$ Ver equação [2.6]).

No trabalho de Coda e Greco (2004) encontra-se uma discussão que permite concluir que essas aproximações não influem significativamente na resposta de barras relativamente esbeltas.

\subsubsection{Exemplo 1 - Flambagem elástica de coluna}

Esse exemplo de validação estático consiste em analisar o fenômeno de instabilidade global de uma coluna com uma das extremidades engastada e submetida a uma força compressiva excêntrica (Ver figura 20). Aplica-se o momento dado por $\frac{M l}{E I}=0.0005$, junto com a força para simular a excentricidade:

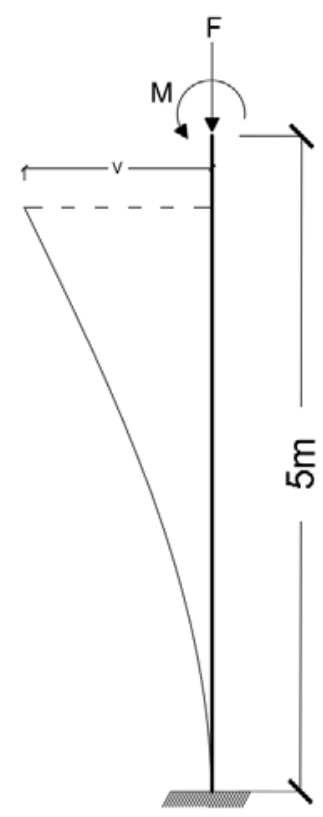

Figura 20 - Esquema para análise de flambagem de coluna. 
Os dados de entrada são apresentados na tabela 4. As dimensões da seção transversal foram adotadas de modo a se obter um índice de esbeltez de 100.

\begin{tabular}{|lr|}
\hline \multicolumn{2}{|c|}{ Dados de entrada } \\
Base [m] & 0,34641 \\
Altura [m] & 0,34641 \\
L [m] & 5,00 \\
E [MPa] & 20 \\
G [MPa] & 10 \\
Passos de carga & 1000 \\
\hline
\end{tabular}

Tabela 4 - Dados de entrada para análise de flambagem elástica de coluna.

Nas figuras 21 compara-se a curva força-deslocamento transversal no plano adimensional obtida analiticamente no trabalho de Goto, Yoshimitsu e Obata (1990) com a resposta numérica obtida, no presente trabalho, empregando 5 elementos finitos de aproximação cubica. Além disso, apresenta-se a curva força-deslocamento longitudinal obtida com a formulação numérica implementada.

Claramente identifica-se o ponto de bifurcação para um valor de $\frac{F L^{2}}{E I}=2.467$, coincidindo com o valor da carga crítica de flambagem elástica obtido pela teoria clássica de Euler. Observa-se a capacidade da formulação numérica para capturar o efeito de encurtamento da coluna. Finalmente, é interessante analisar a configuração de equilíbrio para diferentes valores de carga como mostrado na figura 22, onde se destacam as grandes mudanças de configuração para pequenos incrementos de carga aplicada depois de atingido o ponto de bifurcação. 


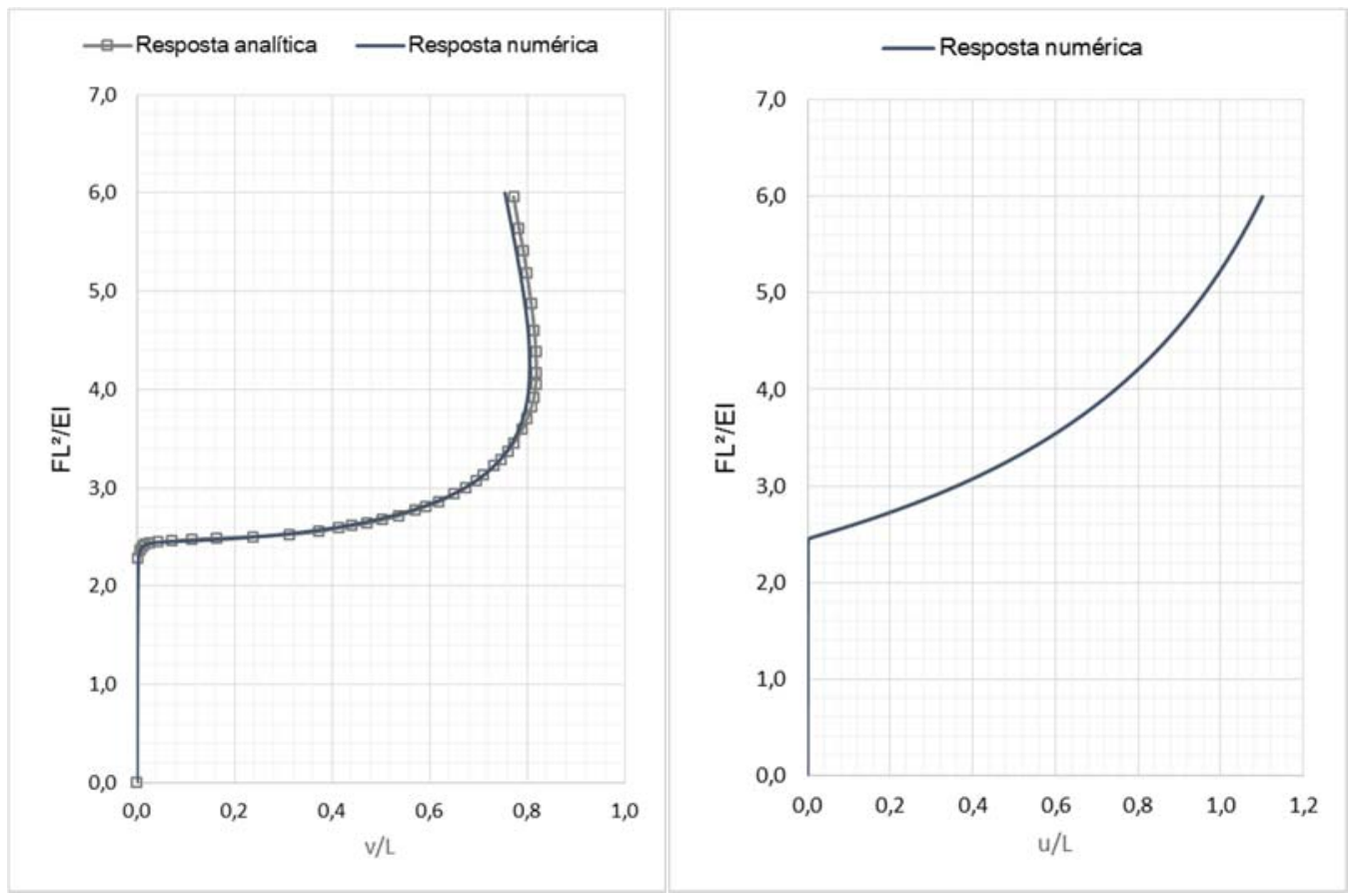

Figura 21 - (a) Curva adimensional força-deslocamento transversal; (b) Curva adimensional força-deslocamento longitudinal.

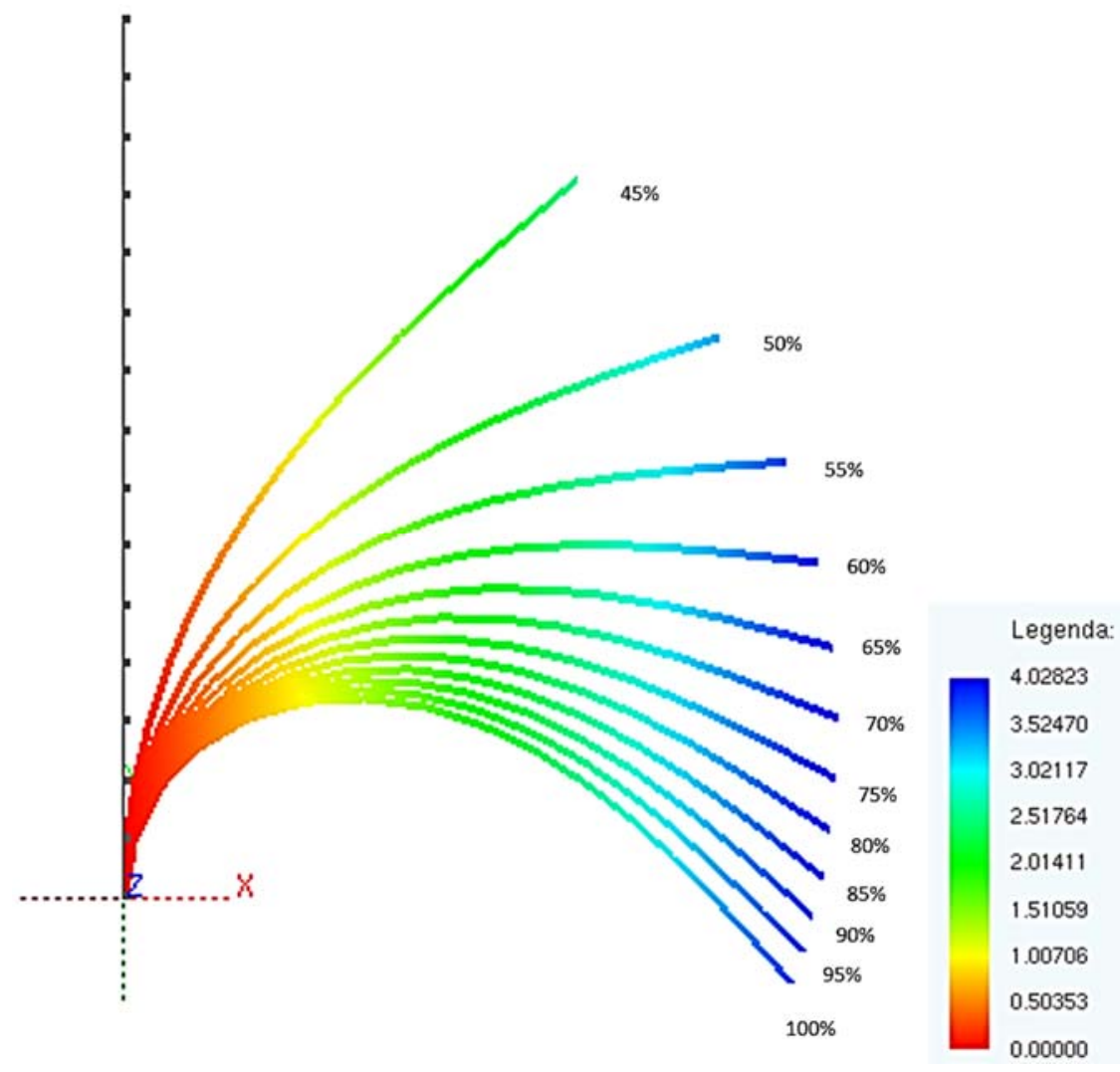

Figura 22 - Evolução do deslocamento transversal $(m)$ e configuração deslocada para diferentes porcentagens de força aplicada. 


\subsubsection{Exemplo 2 - Mecanismo biela-manivela}

Nesse exemplo é testado o comportamento da formulação implementada para análise dinâmica de elementos bidimensionais tipo barra. A estrutura a analisar consiste em um mecanismo clássico biela-manivela (ver figura 23) submetido a valores de momento, aplicado no ponto de apoio da manivela, dado pelas seguintes funções:

$$
\begin{gathered}
M_{1}(t)=0.01 \cdot\left(1-e^{-\frac{t}{0.167}}\right) N \cdot m \\
M_{2}(t)=\left\{\begin{array}{cc}
0.01 \cdot\left(1-e^{-\frac{t}{0.167}}\right) N \cdot m & 0 \leq t \leq 0.7 \\
0 & t>0.7
\end{array}\right.
\end{gathered}
$$

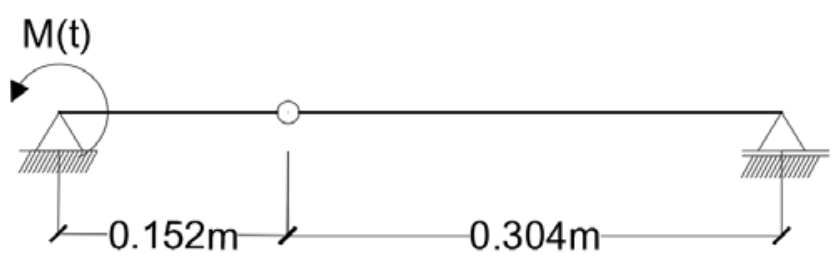

Figura 23 - Esquema do mecanismo biela-manivela.

A manivela tem um comprimento de 0.152 metros e modulo de elasticidade igual a 1.0 GPa, no caso da biela, definiu-se um comprimento de 0.304 metros e um modulo de elasticidade de $0.05 \mathrm{GPa}$. Tanto para a manivela quanto para a biela adotou-se uma largura de $9.06879 \mathrm{~mm}$ e uma altura de $8.66047 \mathrm{~mm}$ e densidade igual a $2770 \mathrm{~kg} / \mathrm{m}^{3}$. Na tabela 5 encontra-se um resumo dos dados de entrada mencionados. 


\begin{tabular}{|lr|}
\hline \multicolumn{2}{|c|}{ Dados de entrada } \\
Inercia $\left[\mathrm{m}^{4}\right]$ & $4,909 \mathrm{E}-10$ \\
Area $\left[\mathrm{m}^{2}\right]$ & $7,854 \mathrm{E}-05$ \\
$\rho\left[\mathrm{kg} / \mathrm{m}^{3}\right]$ & 2770 \\
Base $[\mathrm{mm}]$ & 9,06879 \\
Altura $[\mathrm{mm}]$ & 8,66047 \\
Manivela & \\
$\mathrm{E}[\mathrm{GPa}]$ & 1 \\
$\mathrm{~L}[\mathrm{~m}]$ & 0,152 \\
Biela & \\
$\mathrm{E}[\mathrm{GPa}]$ & 0,05 \\
$\mathrm{~L}[\mathrm{~m}]$ & 0,304 \\
$\Delta \mathrm{t}[\mathrm{s}]$ & $1,00 \mathrm{E}-04$ \\
\hline
\end{tabular}

Tabela 5 - Dados de entrada para análise dinâmica do mecanismo biela-manivela.

O mecanismo foi discretizado utilizando três elementos finitos de aproximação cubica para a manivela e seis elementos para a biela. Em relação aos parâmetros do algoritmo de integração temporal empregou-se o método de Newmark para aceleração média constante.

A figura 24 mostra a posição horizontal do apoio deslizante no decorrer do tempo quando aplicadas as funções de momento descritas anteriormente. As diferenças com os trabalhos de referência devem-se a que um deles adota cinemática de Euler-Bernoulli (ESCALONA et al., 1998) e o outro utiliza a deformação não linear de engenharia (GRECO E CODA, 2006). 


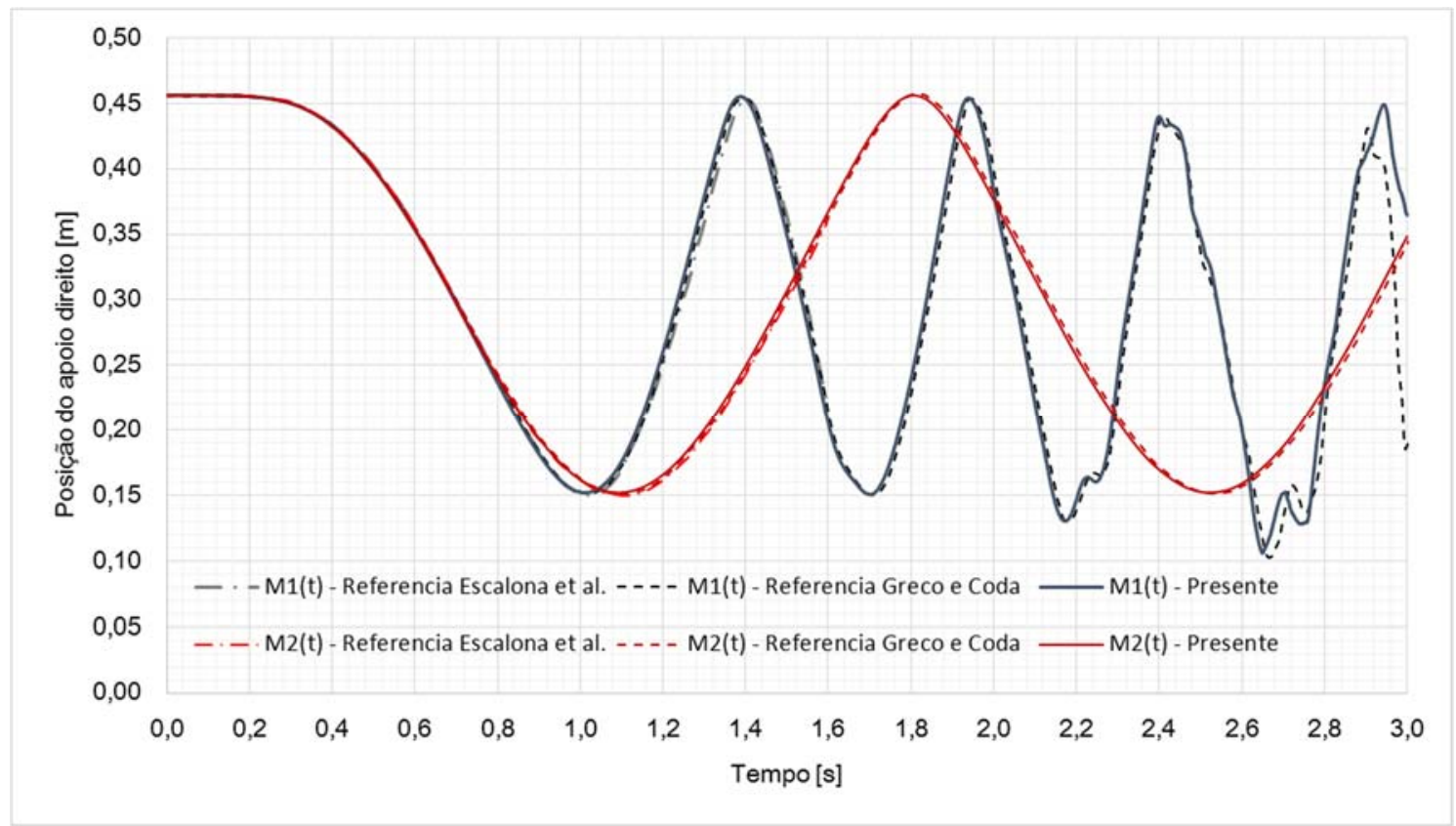

Figura 24 - Histórico da posição horizontal do apoio direito para as funções de momento aplicadas.

$\mathrm{Na}$ figura 25 apresenta-se em mapa de cores o deslocamento horizontal do mecanismo e a configuração deslocada do mesmo para diferentes instantes de tempo ao aplicar a função de momento $\mathrm{M} 2(\mathrm{t})$ evidenciando a capacidade da formulação implementada em representar grandes deslocamentos e deslocamentos como corpo rígido das estruturas.

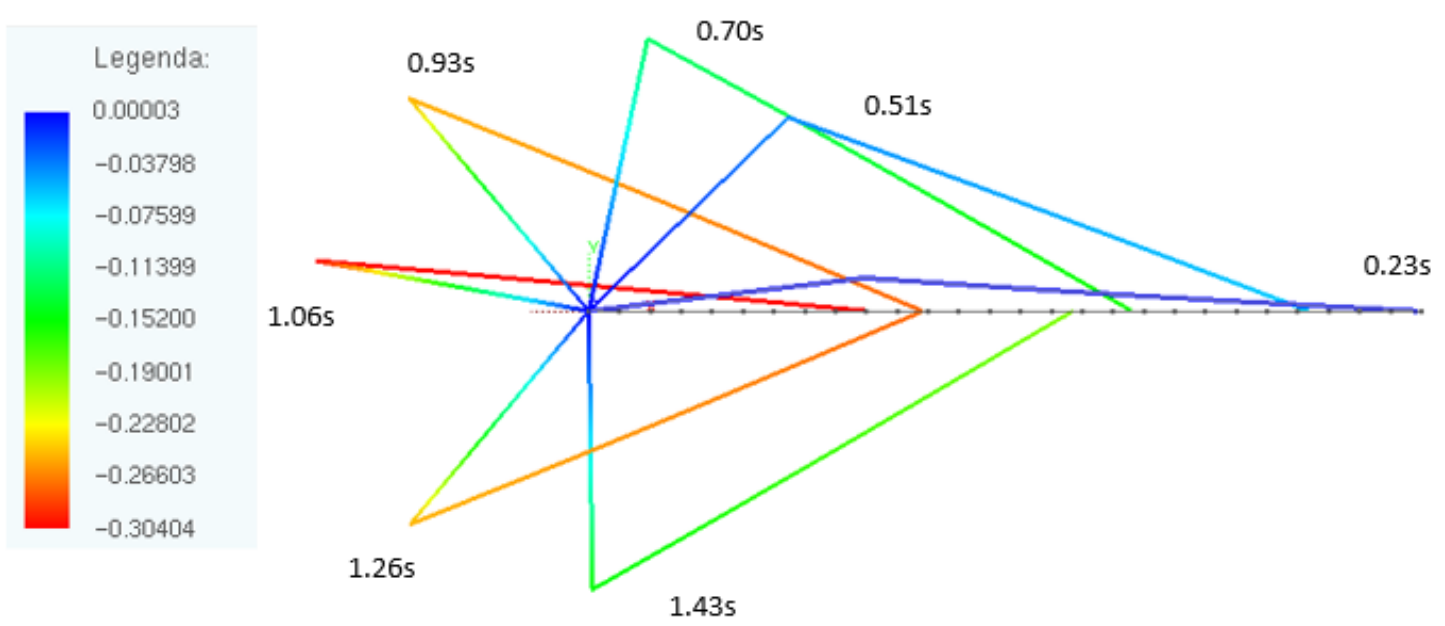

Figura 25 - Evolução do deslocamento horizontal $(m)$ e configuração deslocada para diferentes instantes de tempo ao aplicar a função de momento M2(t). 


\section{LIGAÇÕES DESLIZANTES}

A formulação implementada no presente trabalho encontra-se descrita detalhadamente no trabalho de Siqueira (2016), onde são apresentadas as ligações deslizantes cilíndricas e prismáticas de elementos de pórtico plano. Nesse capítulo, descreve-se de forma breve o seu desenvolvimento matemático.

Uma junta cilíndrica condiciona a posição da extremidade livre de um elemento deslizante, a se deslocar ao longo de um elemento da trajetória, permitindo a existência de uma rotação relativa. No caso da junta prismática, existe transmissão de momento e o grau de liberdade translacional descreve o mesmo comportamento das juntas cilíndricas. Na figura 26, apresentam-se esquematicamente as duas juntas.
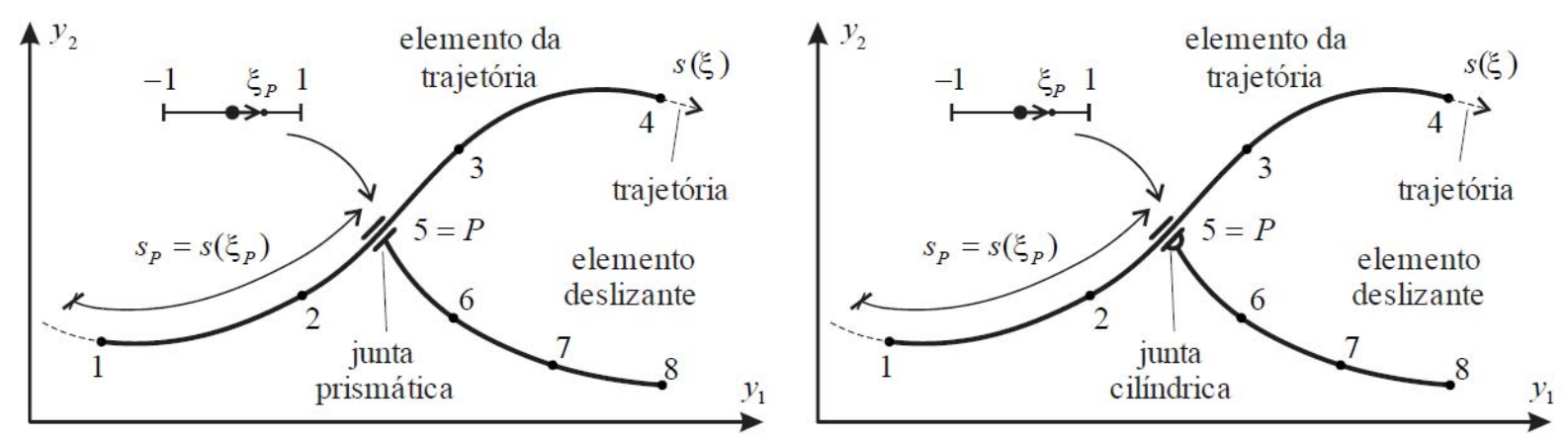

Figura 26 - Descrição esquemática das ligações deslizantes. (a) Ligação prismática. (b) Ligação cilíndrica. Tomado de Siqueira (2016).

Matematicamente, as ligações deslizantes abordadas enquadram como restrições holonomicas escritas de forma implícita, logo deve-se aplicar uma técnica numérica para sua solução. Pela simplicidade da técnica dos multiplicadores de Lagrange na sua formulação e implementação, essa é a técnica aplicada na imposição das restrições cinemáticas. Logo, o algoritmo de solução fica análogo àquele apresentado no desenvolvimento do dispositivo massa/mola/amortecedora no item 2.1.5, isto é, aplicar o método de Newmark da aceleração média na integração temporal da equação de equilíbrio contendo a parcela associada às 
restrições cinemáticas, e o método de Newton-Raphson para dar solução à não linearidade do sistema de equações resultante.

Nota-se ainda que a formulação implementada considera como variável independente o comprimento do elemento da trajetória e não a coordenada adimensional tal como é feito nos trabalhos de Sugiyama, Escalona e Shabana (2003), García-Vallejo et al. (2003) e Lee et al. (2008). Essa é uma característica importante dessa abordagem quando comparada com outras alternativas disponíveis na literatura para tratar ligações deslizantes (BAUCHAU, 2000) (BAUCHAU; BOTTASSO, 2001). As vantagens dessa abordagem se sustentam nas aplicações futuras, já que utilizar a variável curvilínea $S_{P}$ permite a consideração de massas concentradas e a aplicação de forças tangenciais que facilitam a implementação dos modelos de atrito. Além disso, o multiplicador de Lagrange toma o significado físico de força de contato

Nesse capítulo apresenta-se o desenvolvimento matemático necessário para a implementação computacional de juntas cilíndricas e no transcurso do capítulo comentam-se as considerações adicionais para o caso de juntas prismáticas. $O$ item a seguir tem como objetivo explicitar os termos associados à parcela da restrição cinemática a serem adicionados no vetor de forças de desbalanceamento e na matriz hessiana do problema quando for modelada uma ligação deslizante como a descrita nos parágrafos anteriores.

\subsection{Restrição cinemática via multiplicadores de Lagrange}

Nessa abordagem, uma ligação deslizante é definida por dois conjuntos de elementos de pórtico. O primeiro deles descreve a trajetória a ser seguida pelo segundo conjunto de elementos finitos chamados deslizantes. No desenvolvimento

matemático desse item, o símbolo $(\bar{\nabla})$ refere-se aos elementos da trajetória e $(\widehat{\nabla})$ ao elemento deslizante.

Para juntas cilíndricas, a restrição cinemática escreve-se como segue:

$$
\widehat{Y}_{i}^{P}=\bar{y}_{i}^{m}\left(s_{P}(\xi)\right)=\bar{y}_{i}^{m}\left(\xi\left(s_{P}\right)\right)=\bar{y}_{i}^{m}\left(\xi_{P}\right)=\phi_{l}\left(\xi_{P}\right) \bar{Y}_{i}^{l}
$$


Na qual $\widehat{Y}_{i}^{P}$ refere-se à posição do nó $\widehat{P}$ pertencente ao elemento deslizante. Observa-se ainda que a variável $s_{P}$, função da coordenada adimensional $\xi$, define a posição curvilínea e a orientação da seção transversal de um ponto $\bar{P}$ contido na trajetória. Dessa forma, o nó $\widehat{P}$ do elemento deslizante fica restrito a se deslocar sobre o eixo do elemento da trajetória $\bar{y}_{i}^{m}$.

Para o caso de juntas prismáticas ainda deve ser atendida a restrição relacionada ao ângulo de inclinação da seção transversal das barras, escrita como:

$$
\Delta \theta_{P}^{0}=\hat{\theta}_{P}-\phi_{l}\left(\xi_{P}\right) \bar{\theta}_{l}
$$

Onde $\Delta \theta_{P}^{0}$ denota o ângulo formado pelos elementos na configuração inicial, e $\widehat{\theta}_{P}$ representa o ângulo da seção transversal do nó deslizante e $\bar{\theta}_{l}$ o ângulo do nó $l$ pertencente ao elemento ativo da trajetoria.

De forma geral, a restrição cinemática poder ser expressa pela equação a seguir, na qual o índice $i$ varia de 1 a 2 para análise de juntas cilíndricas e de 1 a 3 para análise de juntas prismáticas.

$$
\widehat{Y}_{i}^{P}\left(s_{P}(\xi), \bar{Y}_{i}^{l}\right)=\phi_{l}\left(\xi_{P}\right) \bar{Y}_{i}^{l}+\Delta \theta_{P}^{0} \delta_{i 3}
$$

O funcional da energia total restringe-se, via multiplicadores de Lagrange, ao adicionar a parcela seguinte:

$$
\mathbb{L}\left(\vec{Y}_{L}, s_{P}, \lambda\right)=\lambda_{i}\left[\widehat{Y}_{i}^{P}-\phi_{l}\left(\xi\left(s_{P}\right)\right) \bar{Y}_{i}^{l}-\Delta \theta_{P}^{0} \delta_{i 3}\right]
$$

Onde $\lambda_{i}$ são os multiplicadores de Lagrange e entre colchetes encontra-se a restrição cinemática da equação [3.3]. Desse modo o funcional de energia total do sistema condicionado $\Pi_{L}$ pode ser escrito como:

$$
\Pi_{L}(\vec{Y}, \vec{L})=\Pi(\vec{Y})+\mathbb{L}\left(\vec{Y}_{L}, \vec{L}\right)
$$

Onde $\Pi(\vec{Y})$ refere-se à energia total do sistema (i.e. energia de deformação, cinética, e de amortecimento dos elementos tipo treliça, chapa, e pórtico descritos nos itens $2.1,2.2$ e 2.3, respectivamente) função dos parâmetros nodais $\vec{Y}, \mathbb{L}\left(\vec{Y}_{L}, \vec{L}\right)$ representa a restrição imposta via multiplicadores de Lagrange a qual é função dos 
parâmetros nodais dos elementos envolvidos no deslizamento $\left(\vec{Y}_{L}=\left\{\overrightarrow{\widehat{Y}^{P}}, \overrightarrow{Y^{l}}\right\}\right)$ e dos novos parâmetros $\left(\vec{L}=\left\{\vec{\lambda}, s_{P}\right\}\right)$. Aplicando o princípio de energia total estacionaria chega-se às equações de equilíbrio:

$$
\delta \Pi_{L}=\delta \Pi+\delta \mathbb{L}=0
$$

Já que a variação $\delta \Pi$ foi apresentada nos itens correspondentes a cada elemento tipo, só resta especificar a variação $\delta \mathbb{L}$ respeito às variáveis independentes que definem $\mathbb{L}$ resultando em:

$$
\delta \mathbb{L}=\frac{\partial \mathbb{L}}{\partial \vec{Y}_{L}} \delta \vec{Y}_{(L)}+\frac{\partial \mathbb{L}}{\partial \vec{L}} \delta \vec{L}=\frac{\partial \mathbb{L}}{\partial \bar{Y}_{i}^{\alpha}} \delta \bar{Y}_{i}^{\alpha}+\frac{\partial \mathbb{L}}{\partial \widehat{Y}_{i}^{P}} \delta \widehat{Y}_{i}^{(P)}+\frac{\partial \mathbb{L}}{\partial \lambda_{i}} \delta \lambda_{i}+\frac{\partial \mathbb{L}}{\partial s_{P}} \delta s_{(P)}
$$

Calculando as respectivas derivadas chega-se a:

$$
\delta \mathbb{L}=\vec{\Lambda} \cdot \delta\left\{\vec{Y}_{L}, \vec{L}\right\}=\left\{\delta \bar{Y}_{i}^{\alpha} \delta \widehat{Y}_{i}^{P} \delta \widehat{Y}_{i}^{k} \delta \lambda_{i} \delta s_{P}\right\}\left\{\begin{array}{c}
-\lambda_{i} \phi_{\alpha}\left(\xi_{P}\right) \\
\lambda_{i} \\
0_{k} \\
\widehat{Y}_{i}^{P}-\phi_{l}\left(\xi_{P}\right) \bar{Y}_{i}^{l}-\Delta \theta_{P}^{0} \delta_{i 3} \\
-\lambda_{i} \phi_{l, \xi}\left(\xi_{P}\right) \bar{Y}_{i}^{l} / J_{P}
\end{array}\right\}
$$

Onde $J_{P}$ denota o jacobiano da transformação expresso por:

$$
J\left(\xi_{P}\right)=J_{P}=\sqrt{\left[\phi_{l, \xi}\left(\xi_{P}\right) \bar{Y}_{1}^{l}\right]^{2}+\left[\phi_{l, \xi}\left(\xi_{P}\right) \bar{Y}_{2}^{l}\right]^{2}}
$$

Consequentemente, o vetor de desbalanceamento mecânico fica dado por:

$$
\vec{g}_{L}(\vec{Y}, \vec{L})=M \cdot \ddot{\vec{Y}}+C \cdot \dot{\vec{Y}}+\vec{F} \text { int }-\vec{F} \text { ext }+\vec{\Lambda}=\overrightarrow{0}
$$

E substituindo as aproximações de Newmark obtém-se:

$$
\begin{aligned}
\vec{g}_{L}\left(\vec{Y}_{s+1}, \vec{L}_{s+1}\right) & =\vec{F} \text { int }_{s+1}-\vec{F} \text { ext }_{s+1}+\frac{M}{\mathcal{B} \Delta t^{2}} \cdot \vec{Y}_{s+1}+\frac{\gamma C}{\mathcal{B} t} \cdot \vec{Y}_{s+1}-M \cdot \vec{Q}_{s} \\
& +C \cdot \vec{R}_{s}-\gamma \Delta t C \cdot \vec{Q}_{s}+\vec{\Lambda}_{s+1}=\overrightarrow{0}
\end{aligned}
$$

Percebe-se que as equações [3.10] e [3.11] são idênticas às equações tratadas no desenvolvimento do dispositivo massa/mola/amortecedor. Logo, a linearização do problema pelo método do Newton-Raphson expressa-se como:

$$
\boldsymbol{H}_{\boldsymbol{L}} \cdot\left\{\Delta \vec{Y}_{s+1}, \Delta \vec{L}_{s+1}\right\}=-\vec{g}\left(\vec{Y}_{s+1}^{0}, \vec{L}_{s+1}^{0}\right)
$$


Na qual resolve-se simultaneamente a correção do vetor posição $\vec{Y}$ e das novas variáveis $\lambda_{i}$ e $s_{P}$ e assim poder atualizar o vetor tentativa pela seguinte expressão:

$$
\left\{\begin{array}{l}
\vec{Y}_{s+1} \\
\vec{L}_{s+1}
\end{array}\right\}=\left\{\begin{array}{l}
\vec{Y}_{s+1}^{0} \\
\vec{L}_{s+1}^{0}
\end{array}\right\}+\left\{\begin{array}{l}
\Delta \vec{Y}_{s+1} \\
\Delta \vec{L}_{s+1}
\end{array}\right\}
$$

O cálculo da matriz hessiana do sistema condicionado $\boldsymbol{H}_{L}$ é dado por:

$$
\boldsymbol{H}_{L}=\nabla \vec{g}_{L}=\boldsymbol{H}+\boldsymbol{H}_{L}^{c o n}
$$

$\mathrm{Na}$ qual se observa que $\boldsymbol{H}_{L}$, compõe-se de uma parcela $\boldsymbol{H}$ proveniente do gradiente do vetor de forças internas dos elementos finitos como explicado nos itens 2.1, 2.2 e 2.3 e a outra parcela $\boldsymbol{H}_{L}^{c o n}$ proveniente da derivada segunda da restrição cinemática em relação aos parâmetros nodais $\vec{Y}_{L}$ e às novas variáveis $\vec{\lambda}$ e $s_{P}$ dada por:

$$
\boldsymbol{H}_{\boldsymbol{L}}^{\text {con }}=\frac{\partial \vec{\Lambda}}{\partial\left\{\vec{Y}_{L}, \vec{L}\right\}}=\left[\begin{array}{ll}
\frac{\partial^{2} \mathbb{L}}{\partial \vec{Y}_{L} \partial \vec{Y}_{L}} & \frac{\partial^{2} \mathbb{L}}{\partial \vec{Y}_{L} \partial \vec{L}} \\
\frac{\partial^{2} \mathbb{L}}{\partial \vec{L} \partial \vec{Y}_{L}} & \frac{\partial^{2} \mathbb{L}}{\partial \vec{L} \partial \vec{L}}
\end{array}\right]=\left[\begin{array}{cc}
\mathbf{0} & \boldsymbol{B} \\
\boldsymbol{B}^{\boldsymbol{t}} & \boldsymbol{R}
\end{array}\right]
$$

Para facilitar a identificação dos graus de liberdade nos quais interfere o fato de ter introduzido a restrição cinemática, é conveniente escrever o sistema como:

$$
\boldsymbol{H}_{L}^{\text {con }} \cdot\left\{\begin{array}{c}
\Delta \vec{Y}_{L} \\
\Delta \vec{L}
\end{array}\right\}=\left[\begin{array}{cc}
\mathbf{0} & \boldsymbol{B} \\
\boldsymbol{B}^{\boldsymbol{t}} & \boldsymbol{R}
\end{array}\right] \cdot\left\{\begin{array}{c}
\Delta \vec{Y}_{L} \\
\Delta \vec{L}
\end{array}\right\}
$$

Onde as matrizes $\boldsymbol{B}$ e $\boldsymbol{R}$ ficam dadas por:

$$
\begin{array}{r}
\boldsymbol{B}=\left[\begin{array}{cc}
-\phi_{\alpha}\left(\xi_{P}\right) & -\frac{\lambda_{i} \phi_{\alpha, \xi}\left(\xi_{P}\right)}{J_{P}} \\
1 & 0 \\
0 & 0
\end{array}\right] \\
\boldsymbol{R}=\left[\begin{array}{cc}
0 & -\frac{\phi_{l, \xi}\left(\xi_{P}\right) \bar{Y}_{i}^{l}}{J_{P}} \\
-\frac{\phi_{l, \xi}\left(\xi_{P}\right) \bar{Y}_{i}^{l}}{J_{P}} & H_{S_{P} S_{P}}
\end{array}\right]
\end{array}
$$

E o escalar $H_{S_{P} s_{P}}$ é definido por: 


$$
H_{S_{P} S_{P}}=\lambda_{i} \phi_{l, \xi}\left(\xi_{P}\right) \bar{Y}_{i}^{l}\left(\frac{1}{J_{P}}\right)^{4} \phi_{n, \xi}\left(\xi_{P}\right) \bar{Y}_{k}^{n} \phi_{m, \xi \xi}\left(\xi_{P}\right) \bar{Y}_{k}^{m}-\lambda_{i} \phi_{l, \xi \xi}\left(\xi_{P}\right) \bar{Y}_{i}^{l}\left(\frac{1}{J_{P}}\right)^{2}
$$

Nestas equações, o índice $i$ corresponde às direções (1 a 2 para juntas cilíndricas e de 1 a 3 para juntas prismáticas), $k$ também refere-se às direções mas variando somente de 1 a 2, e, os índices $l, m$ e $n$ representam os nós do elemento ativo da trajetória.

Percebe-se que ao resolver o sistema da equação [3.12] encontra-se a correção da coordenada curvilínea $s_{P}$ para ser atualizada segundo a equação [3.13], no entanto, os termos que compõem o vetor de forças de Lagrange $\vec{\Lambda}$ (equação [3.8]) e a matriz $\boldsymbol{H}_{L}^{\text {con }}$ (equações [3.16] até [3.19]) são definidos em função da variável adimensional $\xi_{P}$. Á visto disso, o item seguinte explica como contornar esse problema, porque apesar das variáveis $s_{P}$ e $\xi_{P}$ estar relacionadas entre si, a função $\xi_{P}=\xi\left(s_{P}\right)$ não é escrita explicitamente, portanto a atualização de $\xi_{P}$ não é direta.

\subsection{Cálculo da variável curvilínea e adimensional}

Como visto no item anterior, no sistema de equações foi adotada $s_{P}$ como variável independente entretanto o cálculo do vetor de forças Lagrange $\vec{\Lambda}$ e a matriz hessiana associada à restrição cinemática depende da variável adimensional $\xi_{P}$ que não é explicitamente escrita no equacionamento. Portanto, o cálculo de $\xi_{P}$ é feito a partir da relação implícita que tem com os valores de $\widehat{Y}_{i}^{P}$ e $\bar{Y}_{i}^{l}$ aplicando a técnica dos mínimos quadrados (SIQUEIRA, 2016), a qual parte da função resíduo definida por:

$$
r_{i}\left(\xi_{P}\right)=\widehat{Y}_{i}^{P}-\phi_{l}\left(\xi_{P}\right) \bar{Y}_{i}^{l}=0_{i}
$$

Nota-se que as coordenadas $\widehat{Y}_{i}^{P}$ e $\bar{Y}_{i}^{l}$ são conhecidas, portanto $r_{i}\left(\xi_{P}\right)$ representa o resíduo quando o valor de $\xi_{P}$ não for a solução. Segundo a técnica, o menor valor do resíduo encontra-se quando a função objetivo atingir o mínimo. Desta forma, define-se como função objetivo o erro quadrático médio: 


$$
p\left(\xi_{P}\right)=\frac{1}{2}\left[r_{i}\left(\xi_{P}\right) r_{i}\left(\xi_{P}\right)\right]=0_{i}
$$

A qual pode ser expandia em série de Taylor truncada na aproximação de primeira ordem, resultando em:

$$
p\left(\xi_{P}\right) \cong p\left(\xi_{P}^{0}\right)+\nabla p\left(\xi_{P}^{0}\right) \Delta \xi_{P}=0
$$

Onde $\xi_{P}^{0}$ refere-se ao valor tentativo da solução. Com a aproximação da equação [3.22], o valor mínimo da função objetivo encontra-se quando $\nabla p\left(\xi_{P}\right)=0$, transformando assim um problema de otimização numa aplicação do método de Newton-Raphson expresso pelas seguintes equações:

$$
\begin{gathered}
\nabla p\left(\xi_{P}\right)=\nabla p\left(\xi_{P}^{0}\right)+\nabla^{2} p\left(\xi_{P}^{0}\right) \Delta \xi_{P}=0 \\
\Delta \xi_{P}=-\frac{\nabla p\left(\xi_{P}^{0}\right)}{\nabla^{2} p\left(\xi_{P}^{0}\right)}
\end{gathered}
$$

Onde:

$$
\begin{gathered}
\nabla p\left(\xi_{P}\right)=\phi_{l, \xi}\left(\xi_{P}\right) \bar{Y}_{i}^{l}\left[\phi_{m}\left(\xi_{P}\right) \bar{Y}_{i}^{m}-\widehat{Y}_{i}^{P}\right] \\
\nabla^{2} p\left(\xi_{P}\right)=\phi_{l, \xi \xi}\left(\xi_{P}\right) \bar{Y}_{i}^{l}\left[\phi_{m}\left(\xi_{P}\right) \bar{Y}_{i}^{m}-\widehat{Y}_{i}^{P}\right]+\left[\phi_{l, \xi}\left(\xi_{P}\right) \bar{Y}_{i}^{l}\right]^{2}
\end{gathered}
$$

E assim, atualizar a variável adimensional $\xi_{P}=\xi_{P}^{0}+\Delta \xi_{P}$ até que o critério de parada for atendido. Tendo determinado o valor de $\xi_{P}$, a transição entre os elementos da trajetória é direta, já que dito valor varia entre \pm 1.0 .

3.3 Considerações adicionais para a modelagem de deslizamento entre elementos sólidos bidimensionais.

Tanto para elementos tipo chapa quanto para elementos tipo pórtico implementaram-se elementos finitos isoparamétricos de aproximação cúbica. Assim, com os nós pertencentes a um mesmo lado de um elemento de chapa define-se um 
elemento pórtico, portanto, prescinde-se de um algoritmo de interpolação que relacione os graus de liberdade dos corpos envolvidos (ver figura 27).

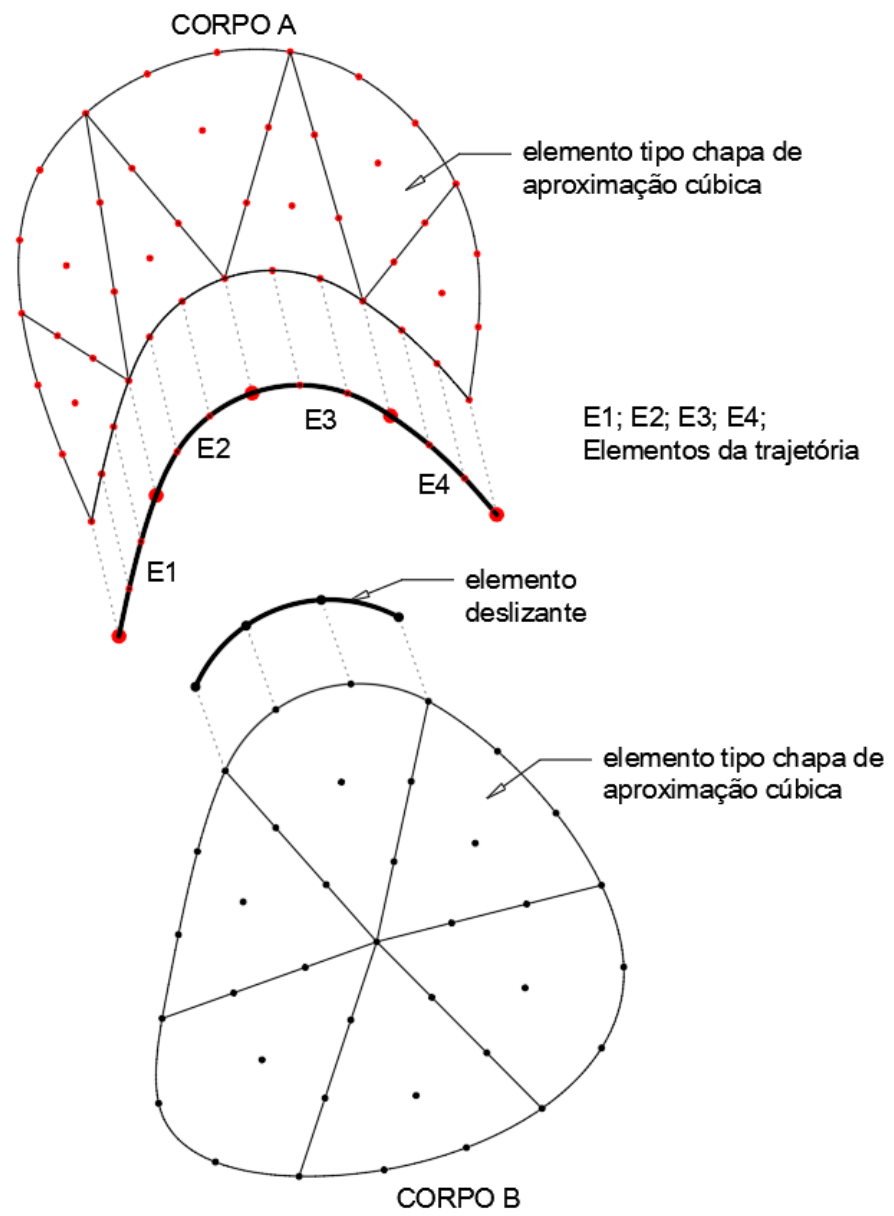

Figura 27 - Acoplamento entre elementos sólidos bidimensionais e elementos de pórtico.

A estratégia descrita aplica-se nas faces do contorno dos corpos que participam no deslizamento, desta forma, todos os nós de um mesmo elemento deslizante ficam restritos a se movimentar sobre o conjunto de elementos que definem a trajetória.

Na figura 27, observa-se ainda que a posição dos nós do elemento deslizante não necessariamente têm que coincidir com a posição dos nós dos elementos da trajetória, no entanto devem estar contidos nela.

Para aproveitar a formulação descrita em Siqueira (2016), deve-se definir uma junta cilíndrica por cada nó deslizante. Por exemplo, no caso da figura 27 
devem-se definir 4 ligações deslizantes com uma mesma trajetória definida pelos elementos E1, E2, E3 e E4.

Além disso, para levar em consideração o fato que mais de um nó deslizante pode se encontrar dentro do domínio de um mesmo elemento da trajetória, o termo $\frac{\partial \mathbb{L}}{\partial \bar{Y}_{i}^{\alpha}}$ deve incluir um somatório. Assim sendo, a equação [3.8] para o cálculo do vetor de forças de Lagrange $\vec{\Lambda}$ reescreve-se como:

$$
\delta \mathbb{L}=\vec{\Lambda} \cdot \delta\left\{\vec{Y}_{L}, \vec{L}\right\}=\left\{\delta \bar{Y}_{i}^{\alpha} \delta \widehat{\mathrm{Y}}_{i}^{P} \delta \widehat{\mathrm{Y}}_{i}^{k} \delta \lambda_{i} \delta s_{P}\right\}\left\{\begin{array}{c}
\sum_{l z=1}^{n l z}-\lambda_{i} \phi_{\alpha}\left(\xi_{P}\right) \\
\lambda_{i} \\
0 k \\
\widehat{\mathrm{Y}}_{i}^{P}-\phi_{l}\left(\xi_{P}\right) \bar{Y}_{i}^{l}-\Delta \theta_{P}^{0} \delta_{i 3} \\
-\lambda_{i} \phi_{l, \xi}\left(\bar{\zeta}_{P}\right) \bar{Y}_{i}^{l} / J_{P}
\end{array}\right\}
$$

$\mathrm{Na}$ qual o índice lz percorre o número de ligações deslizantes.

O equacionamento para o cálculo da matriz hessiana e da variável adimensional mantém-se da forma apresentada nos itens anteriores.

Observa-se ainda que aos elementos de pórtico auxiliares pode ou não ser atribuído um valor para o módulo de elasticidade, quer dizer, que para valores nulos, a rigidez dos corpos fica exclusivamente dependente das propriedades físicas do elemento sólido. Pode-se dizer o mesmo quanto à massa.

\subsection{Exemplo 1 - Flambagem de uma estrutura aplicando força de tração}

O objetivo desse exemplo é mostrar o ótimo funcionamento da formulação apresentada para a análise estática de estruturas com ligações deslizantes. A estrutura a analisar foi resolvida analítica e experimentalmente no trabalho de Zaccaria et al. (2011) e consiste em duas barras flexíveis, de comprimento $L=0.25$ $\mathrm{m}$, alinhadas horizontalmente e unidas por meio de juntas prismáticas a uma barra rígida (ver figura 28). No apoio direito aplica-se uma força horizontal e dada uma perturbação inicial no ângulo da barra rígida apresenta-se simultaneamente o fenômeno de flambagem nas barras horizontais. 


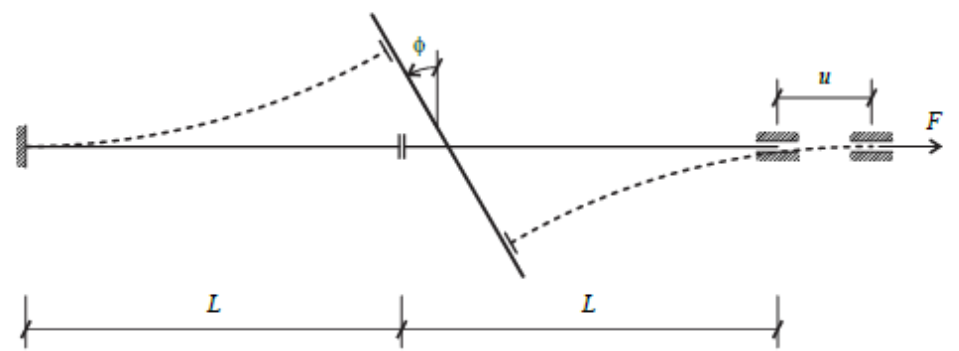

Figura 28 - Esquema da estrutura para análise de flambagem aplicando força de tração. Tomado de Siqueira (2016)

Para a simulação numérica adotou-se uma seção retangular com largura de $25.0 \mathrm{~mm}$ e altura de $1.0 \mathrm{~mm}$ para as barras flexíveis e uma seção quadrada de lado igual a $1.0 \mathrm{~m}$ para a barra rígida. Para todos os elementos empregou-se um modulo de elasticidade de 200.0 GPa e um modulo transversal igual a 100.0 GPa. Cada barra foi discretizada com quatro elementos finitos de aproximação cubica.

Na figura 29 plota-se o ângulo de inclinação da barra rígida com a força adimensional para diferentes valores de perturbação inicial. Já a figura 30 mostra o gráfico do deslocamento horizontal do apoio direito e a força adimensional. Percebese que os resultados numéricos convergem para a resposta analítica na medida que o ângulo de perturbação inicial adotado se aproxima a zero. 


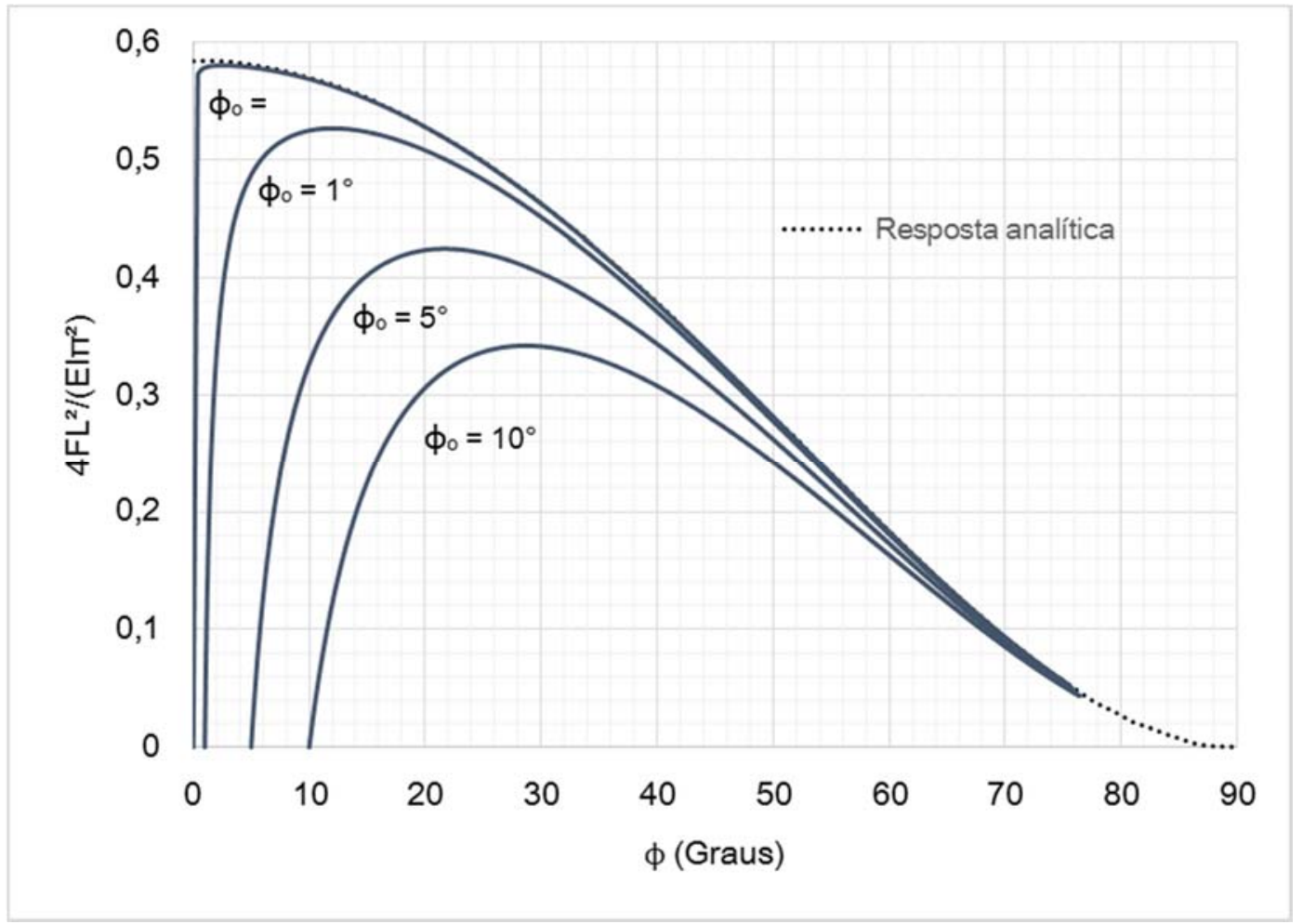

Figura 29 - Evolução do ângulo de inclinação da barra rígida.

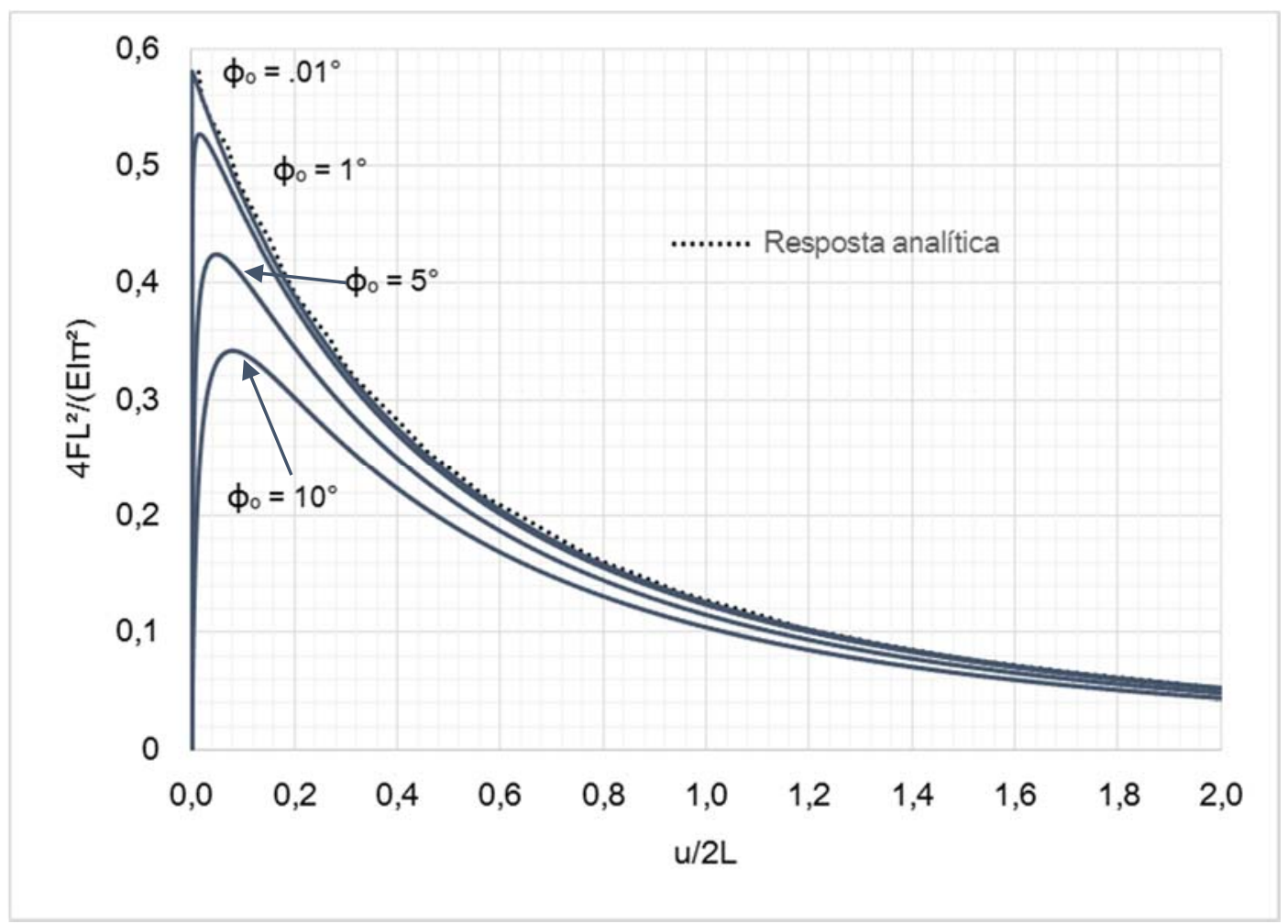

Figura 30 - Evolução do deslocamento horizontal do apoio direito. 


\subsection{Exemplo 2 - Mecanismo de retorno rápido.}

No presente exemplo apresenta-se o mecanismo estudado no trabalho de Bauchau (2000) que consiste em um braço $A B$ de 1.0 metros de comprimento ligado em um extremo a um apoio fixo e no outro conecta-se ao elemento NA de 0.25 metros de comprimento. O movimento do sistema gera-se pela imposição de uma velocidade angular constante de $5 \pi \mathrm{rad} / \mathrm{s}$ no elemento RS de 0.20 metros de comprimento que se conecta ao braço $A B$ por meio de uma junta cilíndrica localizada no nó S (Ver figura 31).

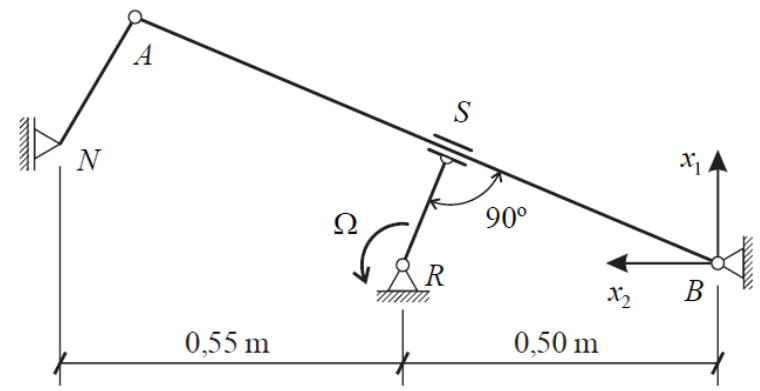

Figura 31 - Esquema do mecanismo de retorno. Tomado de Siqueira (2016).

Empregaram-se 24 elementos finitos com modulo de elasticidade de 47.04 GPa para discretizar o braço $A B$ e 2 elementos finitos com modulo de elasticidade de $47.04 \times 10^{6} \mathrm{GPa}$ para cada umas das barras restantes. Todas as barras têm uma densidade de $1724.80 \mathrm{~kg} / \mathrm{m}^{3}$ e uma seção transversal quadrada de 5.98 centímetros. Além disso, foram adicionadas duas massas concentradas nos nós $\mathrm{N}$ e $\mathrm{S}$ com valor de $0.31 \mathrm{~kg}$ e $2.50 \mathrm{~kg}$, respectivamente. Adotou-se um passo de tempo de $2 \times 10^{-03}$ segundos e foram simulados 3 ciclos.

As figuras seguintes apresentam os resultados obtidos para o terceiro ciclo da análise. Na figura 32 plota-se a velocidade vertical do ponto $\mathrm{N}$ e na figura 33 mostrase a evolução da deflexão do braço no nó $A$.

Como mencionado no trabalho de Siqueira (2016) presume-se que a difereça de fase entre as curvas deve-se aos algoritmos de integração temporal empregados, dado que na formulação implementada no presente trabalho utiliza-se o algoritmo de 
Newmark da aceleração média enquanto em Bauchau (2000) emprega-se um integrador baseado no decaimento da energia total.

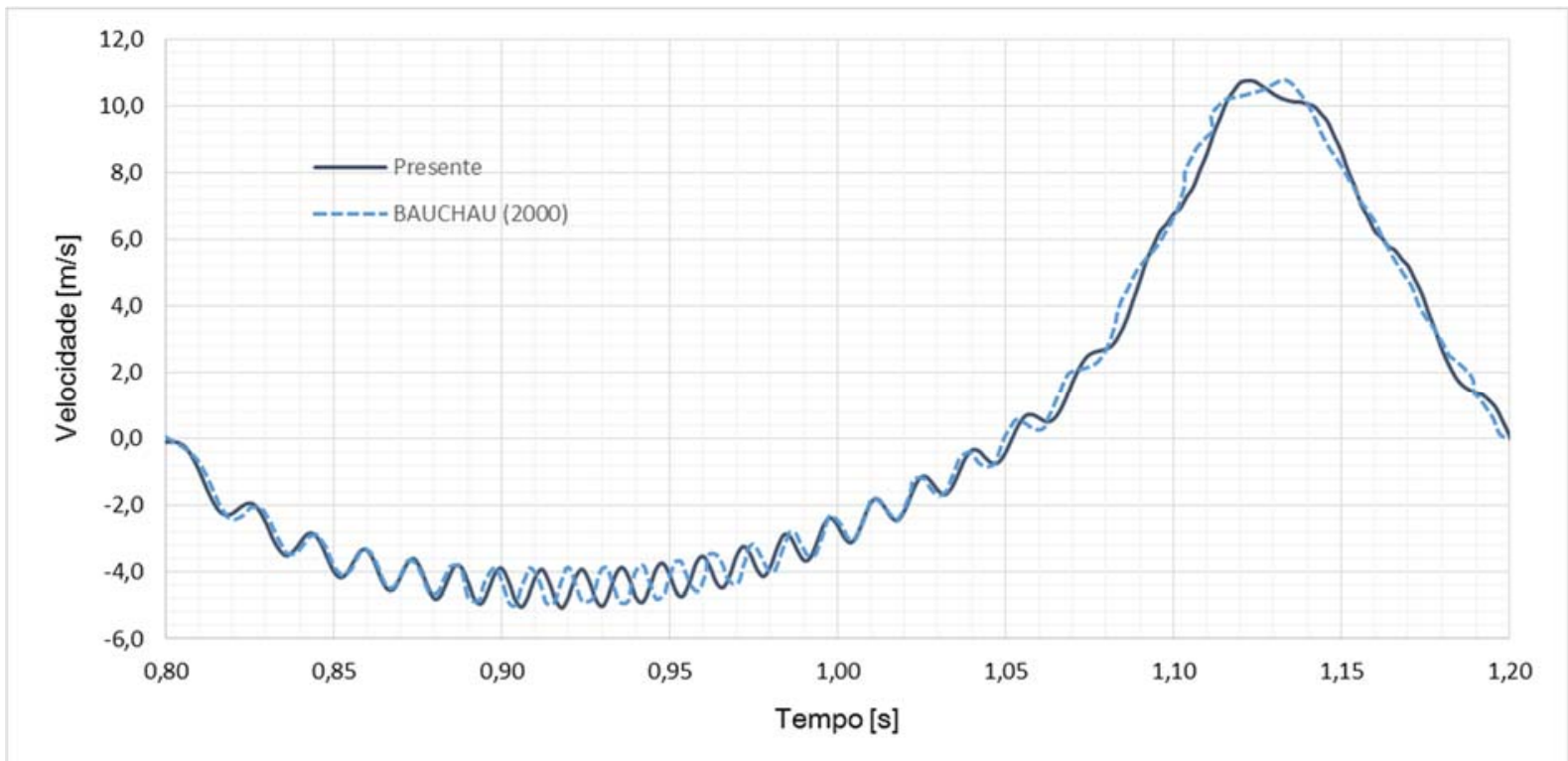

Figura 32 - Velocidade vertical do nó N.

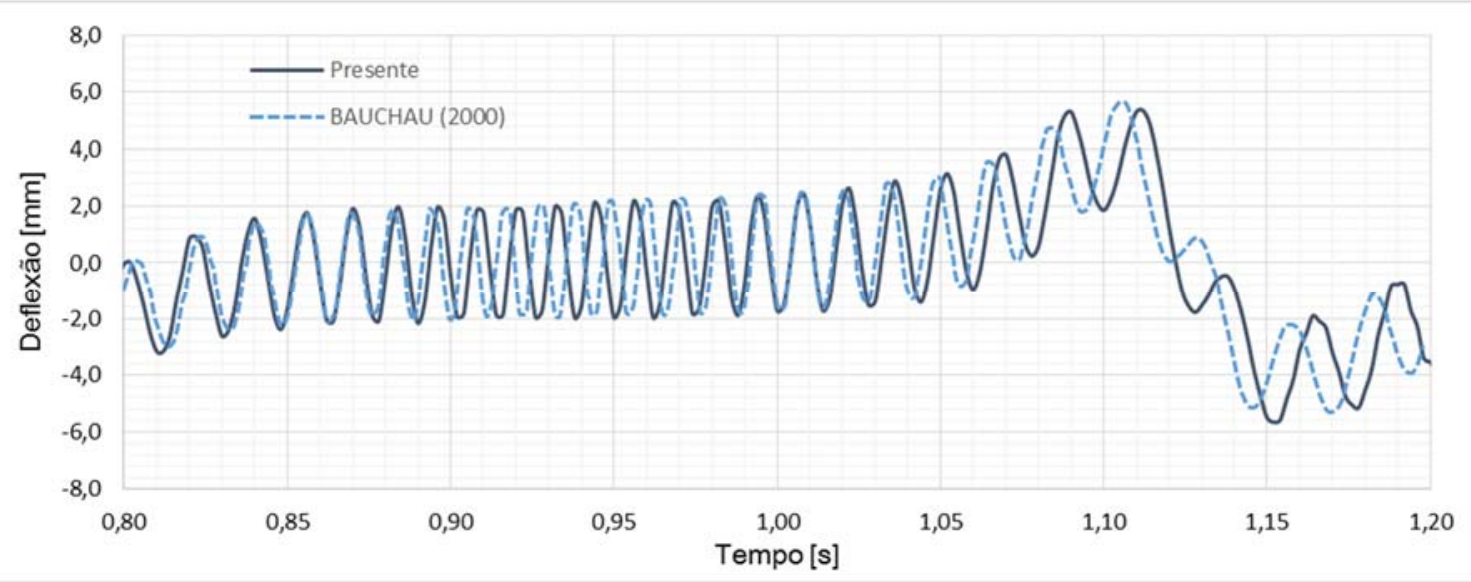

Figura 33 - Deflexão do braço no ponto A. 


\section{APLICAÇÕES: MODELAGEM DE SISTEMAS DE CONTROLE DE VIBRAÇÃO}

No presente capítulo estuda-se o comportamento de uma estrutura plana em elementos pórtico que representa um edifício de 5 andares, 15 metros de altura e 6 metros de largura, submetido a um movimento de base. Desconsidera-se a parcela de amortecimento.

Todos os elementos têm seção transversal quadrada de $0.50 \times 0.50$ metros, modulo de elasticidade de 20GPa e modulo transversal de 10GPa. Os pilares têm uma densidade de $2400 \mathrm{~kg} / \mathrm{m}^{3}$ e com o objetivo de levar em consideração o efeito dinâmico de cargas sobrepostas, atribui-se às vigas uma densidade de 15168.2 $\mathrm{kg} / \mathrm{m}^{3}$. Cada pilar discretizou-se com 3 elementos finitos e cada viga com 6 elementos finitos de aproximação cúbica.

A análise global consiste em uma análise estática preliminar na qual aplica-se um carregamento distribuído de $34.0 \mathrm{kN} / \mathrm{m}$ sobre as vigas e subsequentemente é feita uma análise dinâmica aplicando os deslocamentos de base descritos na figura 34 e tomando um delta de tempo de 5x10-03 segundos que coincide com a discretização temporal do registro do sismo. Assim sendo, a análise dinâmica parte da condição deformada da estrutura devida ao carregamento estático.

A estrutura analisa-se sob as seguintes condições:

(1) estrutura engasta na base sob componente horizontal e vertical do sismo.

(2) estrutura isolada na base com dispositivos FPS. (Caso a) Considera-se as componentes horizontal e vertical do sismo; (Caso b) Considera-se somente a componente horizontal do sismo.

(3) Estrutura com TMD sob componente horizontal e vertical do sismo.

(4) Estrutura com TMD e dispositivos FPS sob componente horizontal e vertical do sismo.

O movimento da base representa um terremoto real. Os dados de deslocamento foram obtidos da base de dados web do centro de pesquisa 'Pacific 
Earthquake Engineering Research Center' (http://ngawest2.berkeley.edu/spectras/ 1822/searches/1605/edit) 5 e correspondem a um terremoto conhecido como 'Superstition Hills' com magnitude de 6.2 que ocorreu o dia 23 de novembro de 1987 no oeste de 'Imperial Valley', perto da cidade de Westmorland, Califórnia, Estados Unidos. ${ }^{6} \mathrm{Na}$ seguinte figura, apresenta-se o gráfico de deslocamentos na direção horizontal e vertical.

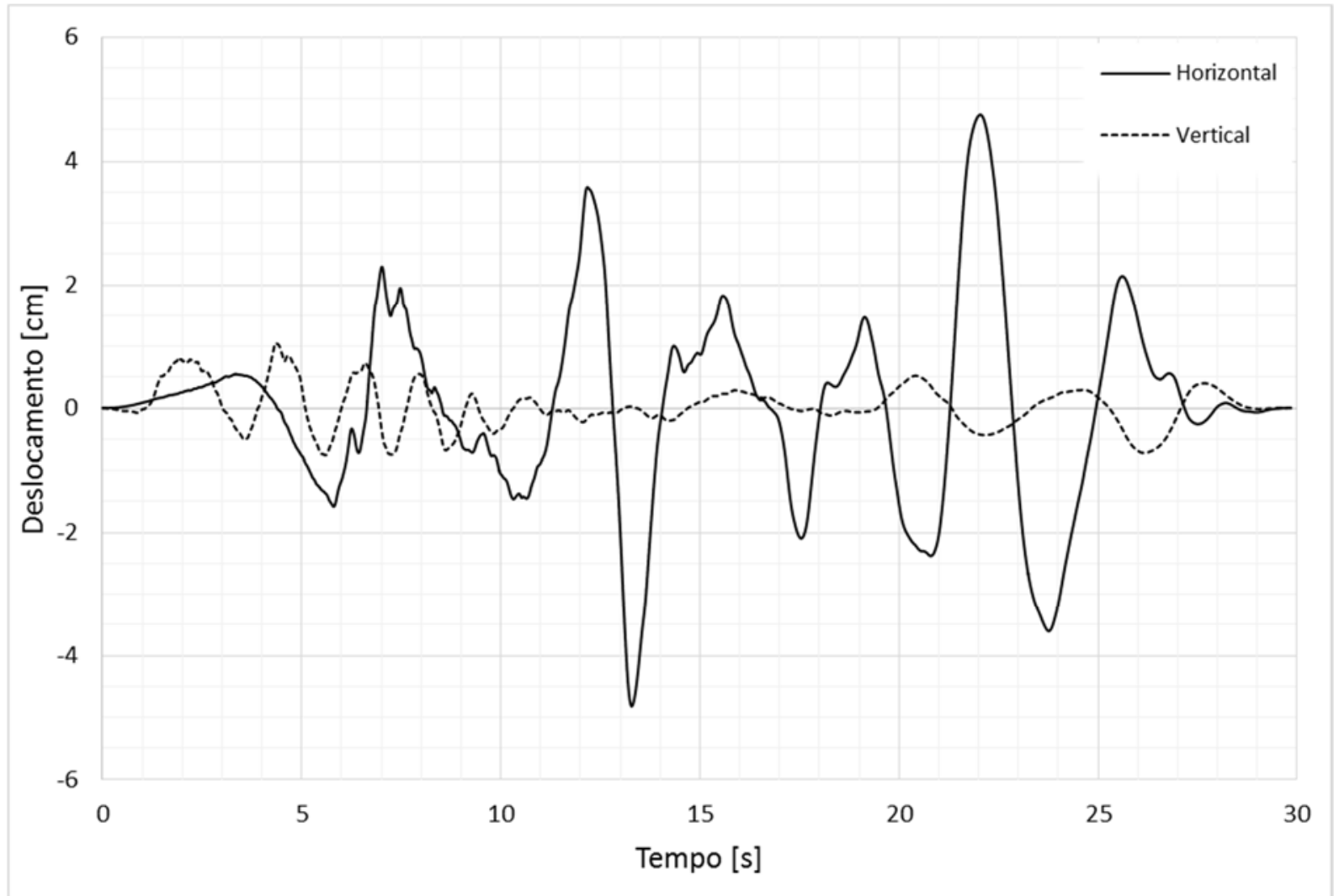

Figura 34 - Terremoto 'Superstition Hills, 1987'. Componente horizontal e vertical dos deslocamentos.

\subsection{Estrutura engastada na base submetida a terremoto.}

$\mathrm{Na}$ tabela 6 apresentam-se os autovalores, as frequências angulares, os períodos e as frequências de vibração dos primeiros 10 modos de vibração da

5 Consultado em Fev 2017.

6 Fonte: Preliminary Report: Surface Rupture Superstition Hills Earthquake. Disponível em: ftp://ftp.consrv.ca.gov/pub/dmg/pubs/cg/1988/41_04.pdf. Consultado em Fev 2017. 
estrutura analisada. As figuras 35 apresentam as formas modais dos primeiros 3 modos de vibração.

\begin{tabular}{|ccccc|}
\hline $\begin{array}{c}\text { Modo } \\
\text { Número }\end{array}$ & $\begin{array}{c}\text { Autovalor } \\
{\left[\mathrm{rad}^{2} / \mathbf{s e g}^{2}\right]}\end{array}$ & $\begin{array}{c}\text { FreqCirc } \\
{[\mathrm{rad} / \mathrm{seg}]}\end{array}$ & $\begin{array}{c}\text { Periodo } \\
{[\mathbf{s e g}]}\end{array}$ & $\begin{array}{c}\text { Frequencia } \\
{[\mathrm{cps}]}\end{array}$ \\
1 & 71,9070 & 8,4798 & 0,7410 & 1,3496 \\
2 & 764,2966 & 27,6459 & 0,2273 & 4,4000 \\
3 & 2717,4876 & 52,1295 & 0,1205 & 8,2967 \\
4 & 4332,3617 & 65,8207 & 0,0955 & 10,4757 \\
5 & 5490,1490 & 74,0955 & 0,0848 & 11,7927 \\
6 & 5991,7852 & 77,4066 & 0,0812 & 12,3196 \\
7 & 6513,4582 & 80,7060 & 0,0779 & 12,8448 \\
8 & 6581,9215 & 81,1290 & 0,0774 & 12,9121 \\
9 & 6966,0191 & 83,4627 & 0,0753 & 13,2835 \\
10 & 11410,9768 & 106,8222 & 0,0588 & 17,0013 \\
\hline
\end{tabular}

Tabela 6 - Modos de vibração.
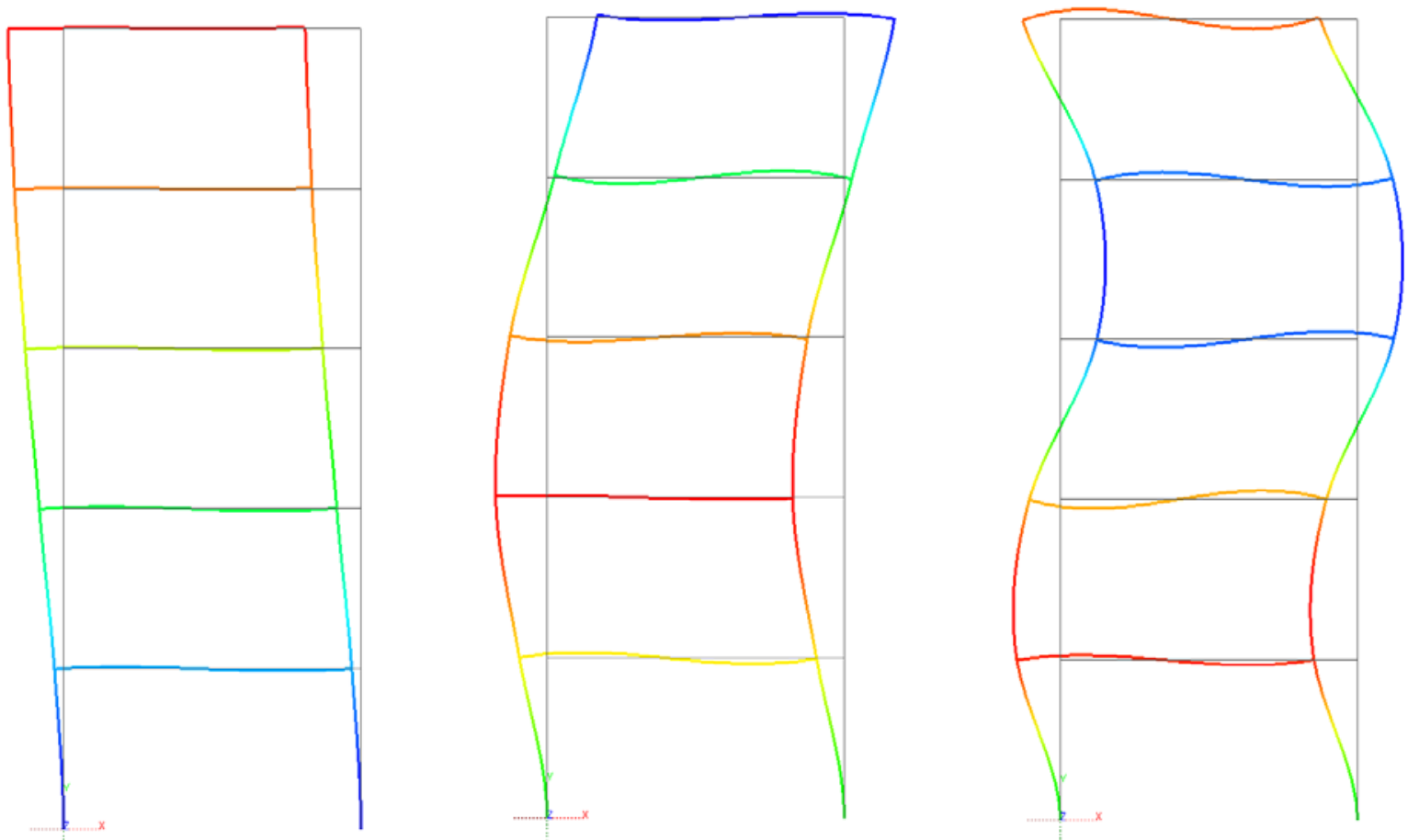

Figura 35 - - (a) Modo 1. (b) Modo 2. (c) Modo 3. 
Nas seguintes figuras apresentam-se as configurações deformadas após a aplicação das forças estáticas e para diferentes instantes de tempo durante a análise dinâmica. A escala de cores mostra os valores de deslocamento horizontal da estrutura.

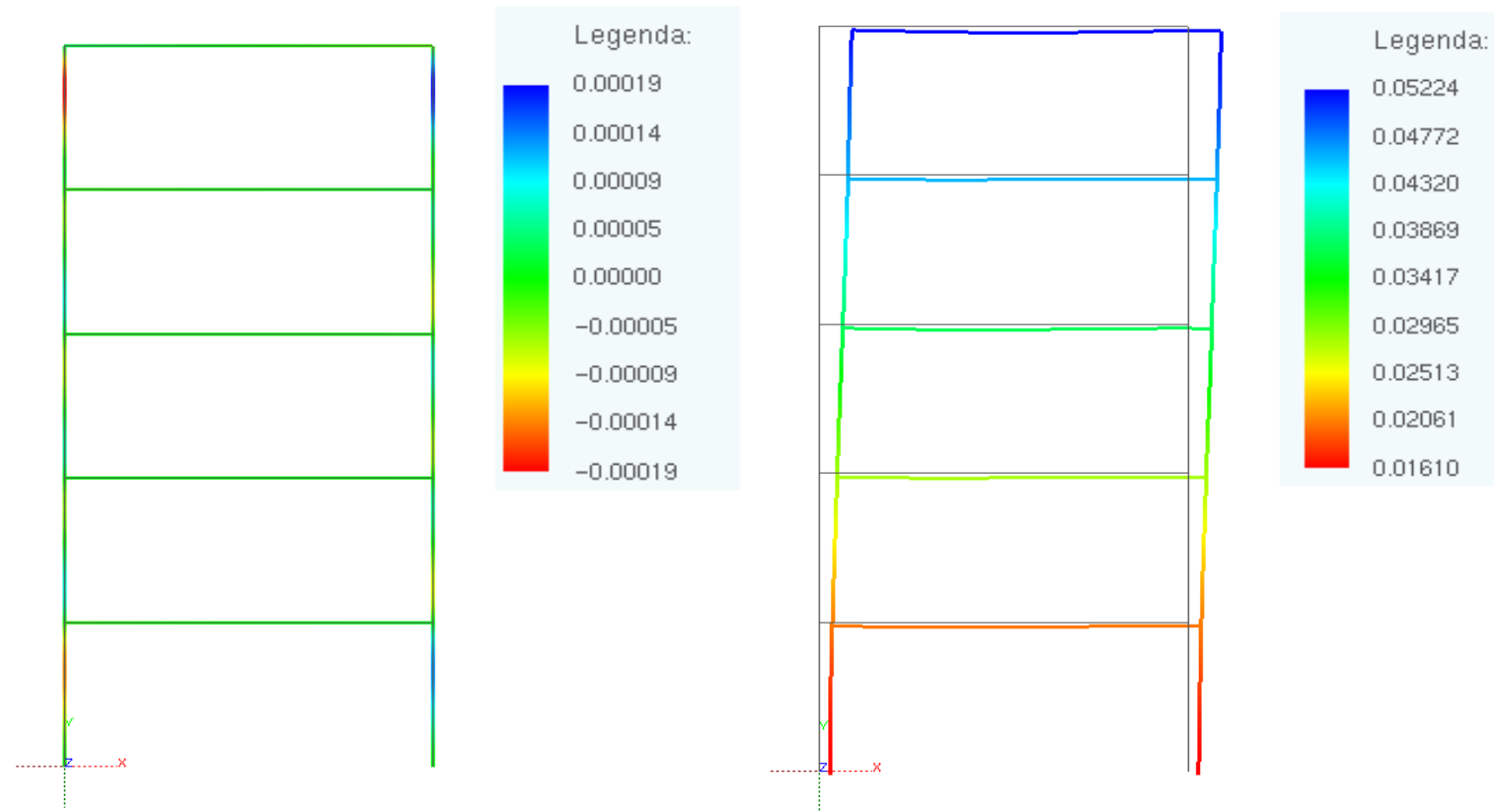

Figura 36 - Estrutura deformada (a) Carregamento estático. (b) análise dinâmica t = 7.19 [s]
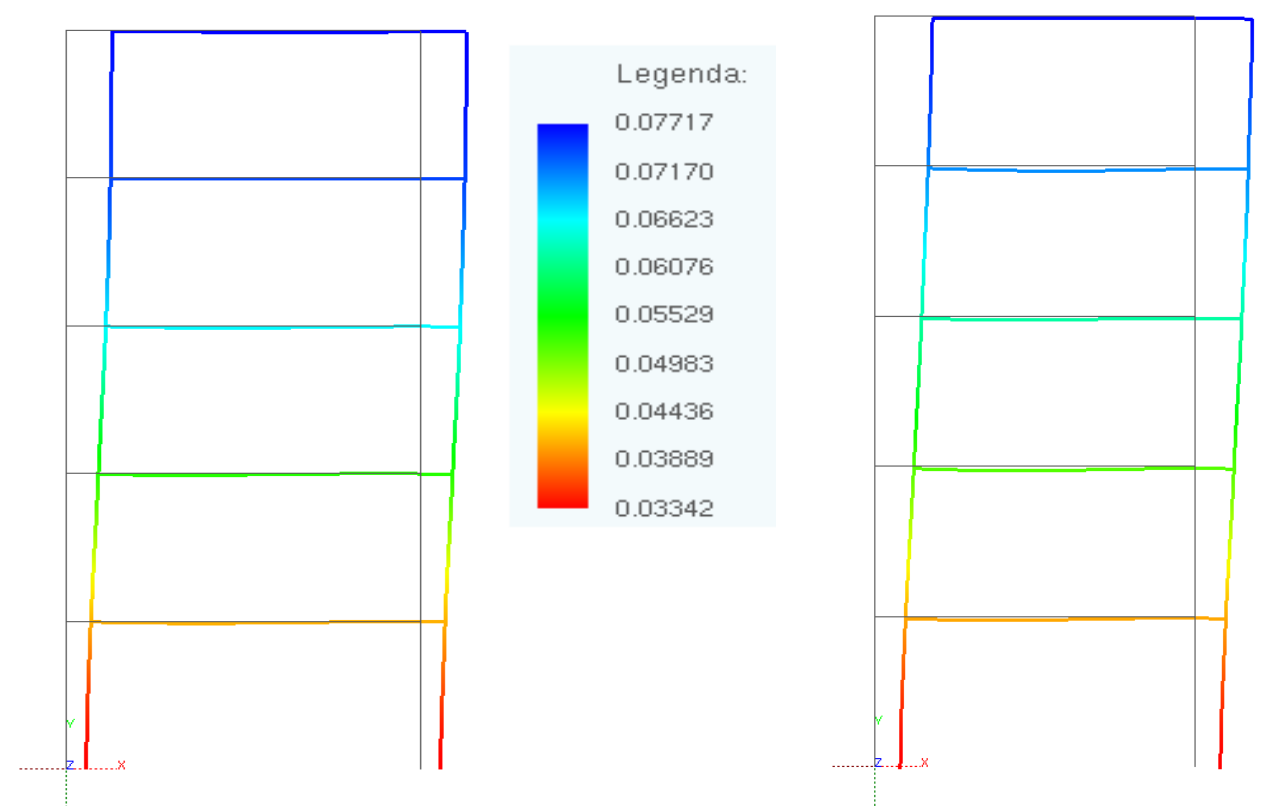

Figura 37 - Análise dinâmica $(a) t=12.36[s](b) t=21.99$ [s] 
Nas figuras 38 e 39 apresentam-se as respostas estruturais em termos de deslocamento e aceleração horizontal do topo da estrutura, respectivamente. Observa-se um valor de deslocamento máximo de $10.77 \mathrm{~cm}$, e uma aceleração máxima $0.873 \mathrm{~g}$. Além disso, a partir da figura 38 estima-se um período de vibração aproximadamente de 0.72 segundos.

Com o objetivo de analisar os deslocamentos relativos, na figura 40 plota-se o deslocamento horizontal nos pontos de ligação entre o pilar esquerdo e as vigas.

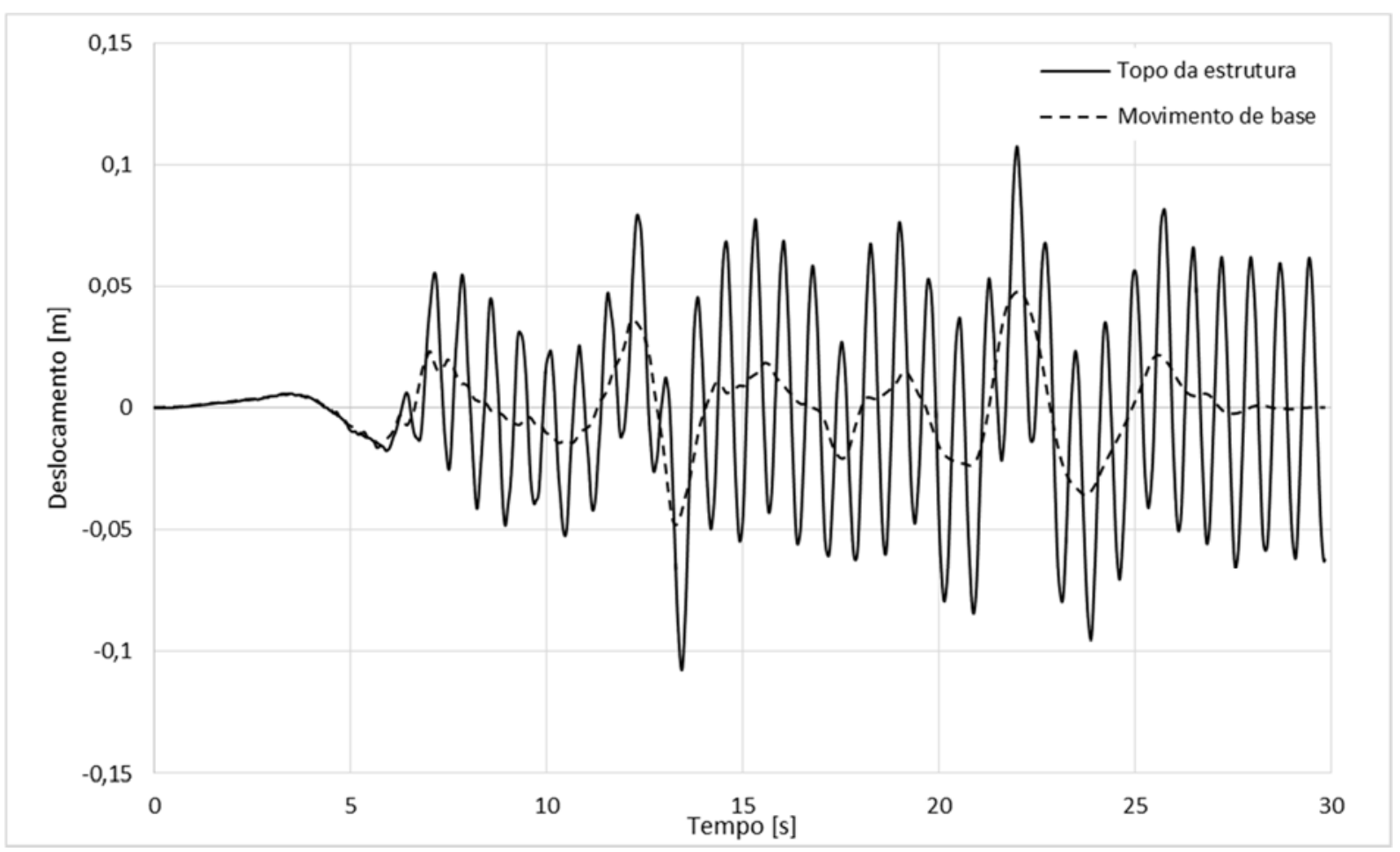

Figura 38 - Deslocamento horizontal. 


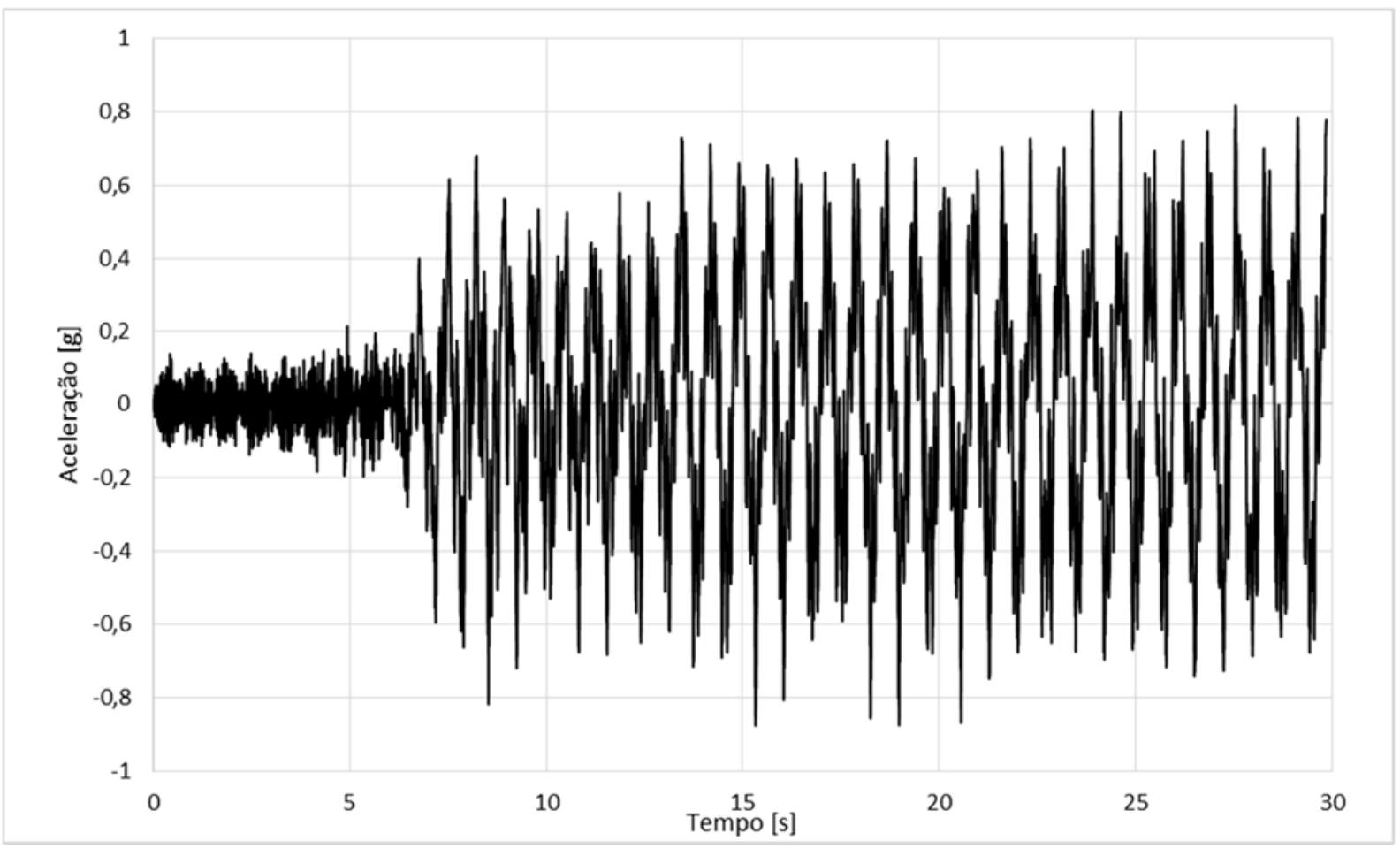

Figura 39 - Aceleração horizontal o topo da estrutura (g).

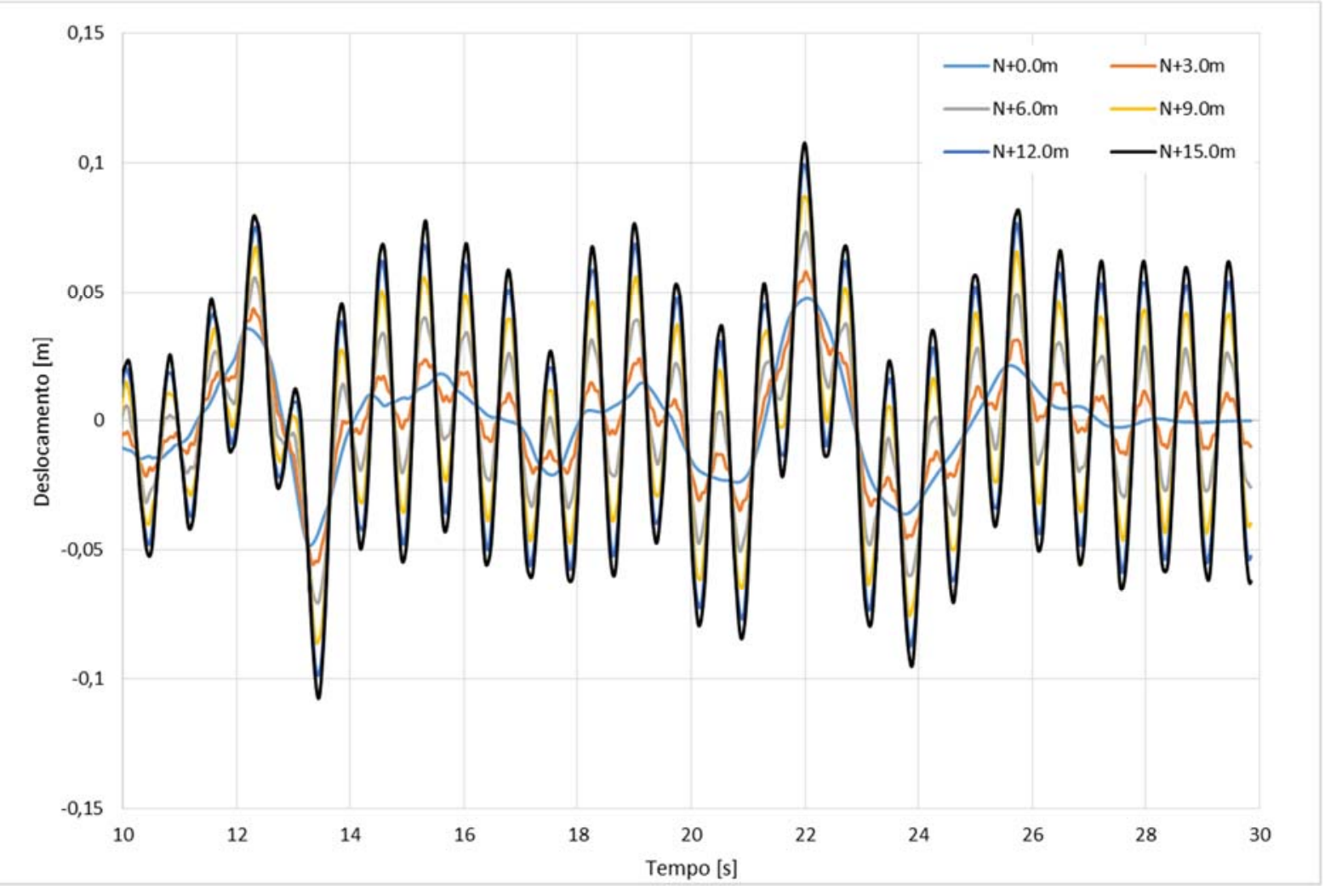

Figura 40 - Deslocamento horizontal do pilar do eixo esquerdo. 
4.2 Estrutura isolada na base com dispositivos FPS.

O dispositivo selecionado apresenta-se no trabalho de Zayas et al. (1990). As propriedades geométricas ilustram-se na figura 41. Destaca-se a largura da superfície côncava e a largura do deslizador articulado, 19" e 6", respectivamente. Capacidade de deslizamento lateral de 6.5" e raio de curvatura de 49" que corresponde a um período de deslizamento de 2.25 segundos.

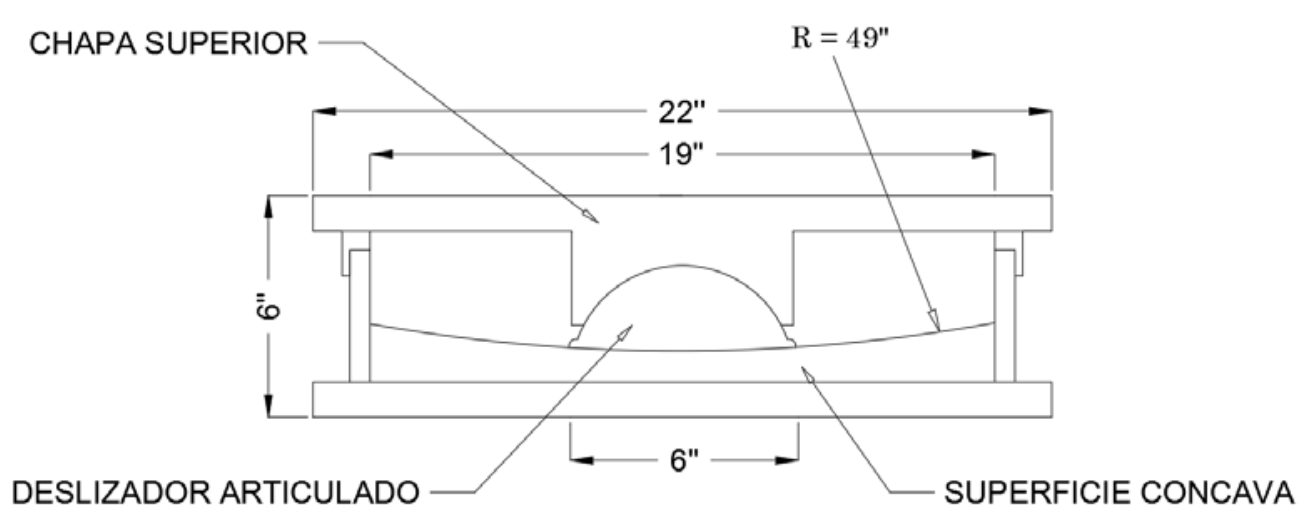

Figura 41 - Detalhe do dispostivo FPS selecionado.

$\mathrm{Na}$ simulação numérica de cada dispositivo FPS empregaram-se 30 elementos triangulares de aproximação cúbica com uma largura de 0.50 metros. Adotou-se densidade de $7850 \mathrm{~kg} / \mathrm{m}^{3}$. Modulo de elasticidade de $200 \mathrm{GPa}$ e modulo transversal de $100 \mathrm{GPa}$. Utilizou-se o gerador de malha 'GMsh' (GEUZAINE; REMACLE, 2009).

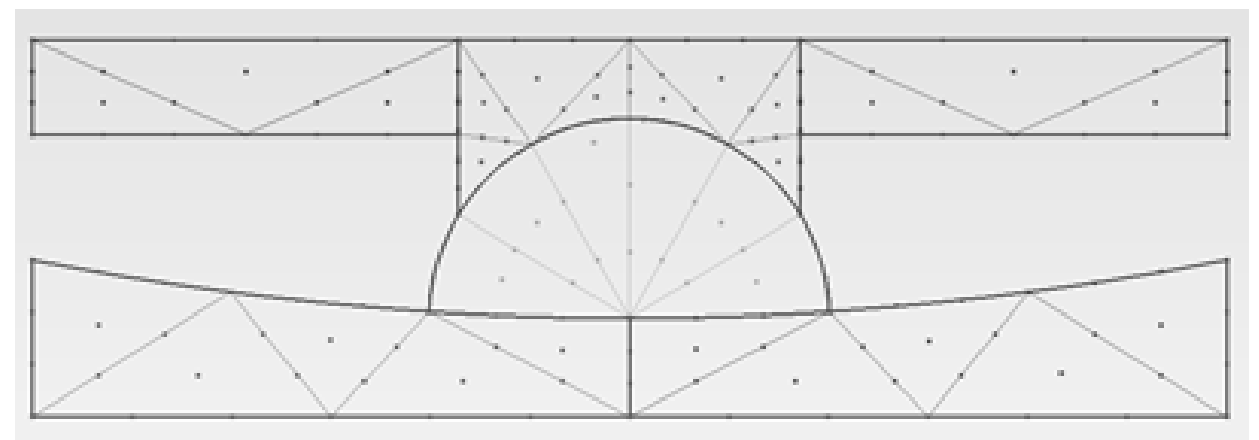

Figura 42 - Discretização do dispositivo FPS. 
Em relação ao deslizador, observa-se que está articulado na chapa superior, desta forma, ele pode girar livremente adaptando-se à curvatura da superfície côncava e evitando a concentração de tensões de contato (ver figura 43). Além disso, garante-se a horizontalidade da chapa superior, incluso, na fase excêntrica do movimento.

Portanto, simular cada dispositivo FPS implica a consideração de dois interfaces de deslizamento, isto é, interface chapa superior - deslizador articulado e chapa inferior - deslizador articulado.

Como mencionado no item 3.3, a estratégia para resolver o problema de deslizamento consiste em criar elementos de pórtico auxiliares nas faces de deslizamento dos sólidos (ver figura 43). Para esses elementos atribuíram-se as seguintes propriedades geométricas e físicas: altura $1 \mathrm{~cm}$, largura $50 \mathrm{~cm}$, densidade $7850 \mathrm{~kg} / \mathrm{m}^{3}$, módulo de elasticidade 200GPa e módulo transversal 100GPa.
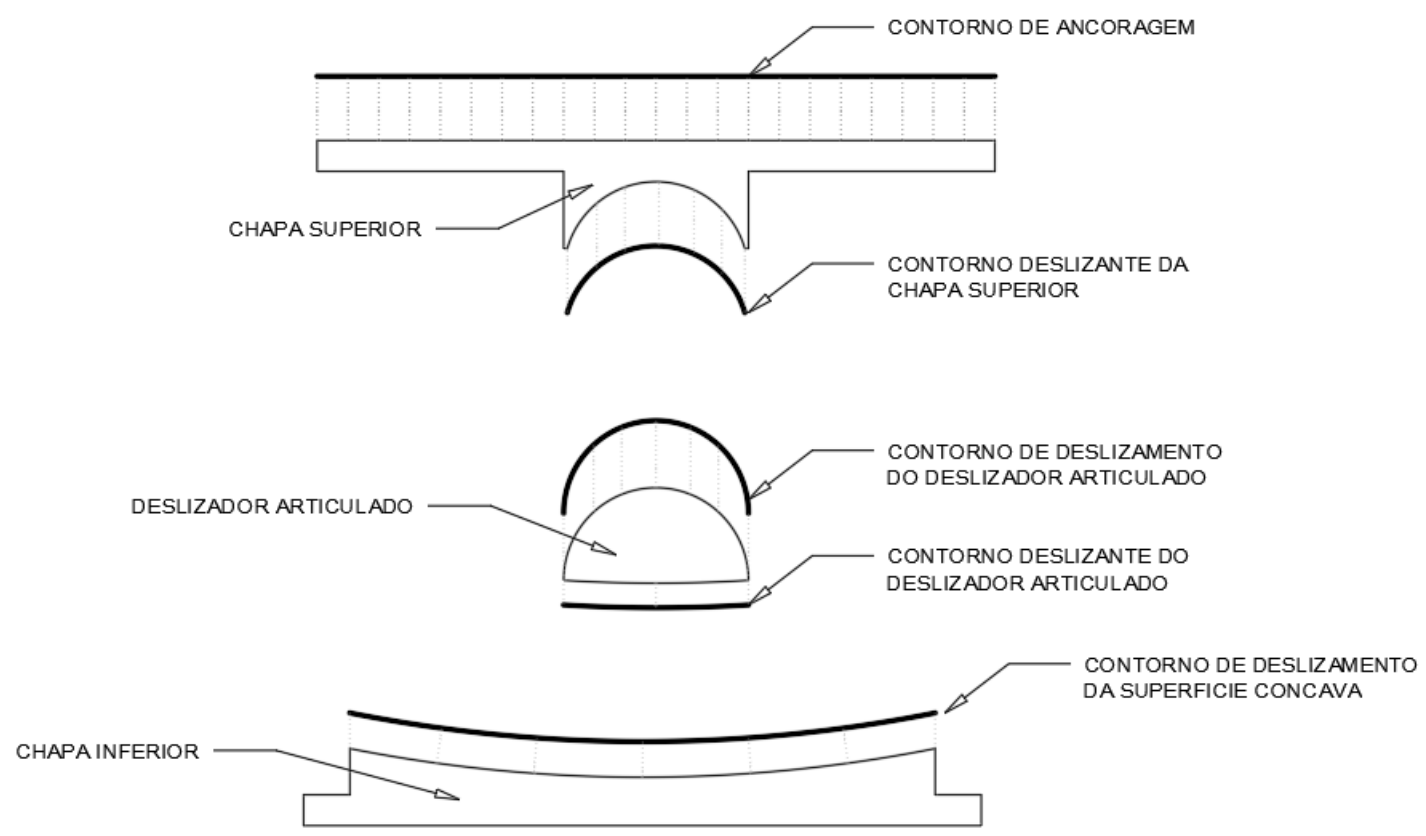

Figura 43 - Descrição gráfica do acoplamento dos elementos de pórtico nas faces deslizantes dos sólidos bidimensionais. 
Comenta-se ainda que os deslocamentos prescritos pelo terremoto aplicaramse nos nós pertencentes à base do elemento sólido chamado de chapa inferior e para garantir a transmissão de momento entre os pilares do pórtico e a chapa superior, utilizaram-se elementos de pórtico de densidade nula no contorno de ancoragem (ver figura 43) e atribuíram-se as seguintes propriedades: altura $50 \mathrm{~cm}$, largura $50 \mathrm{~cm}$, módulo de elasticidade $200 \times 10^{6} \mathrm{GPa}$ e módulo transversal de $100 \times 10^{6}$ GPa.

$\mathrm{Na}$ figura 44 apresenta-se a malha de elementos finitos resultante das considerações descritas nos parágrafos anteriores.

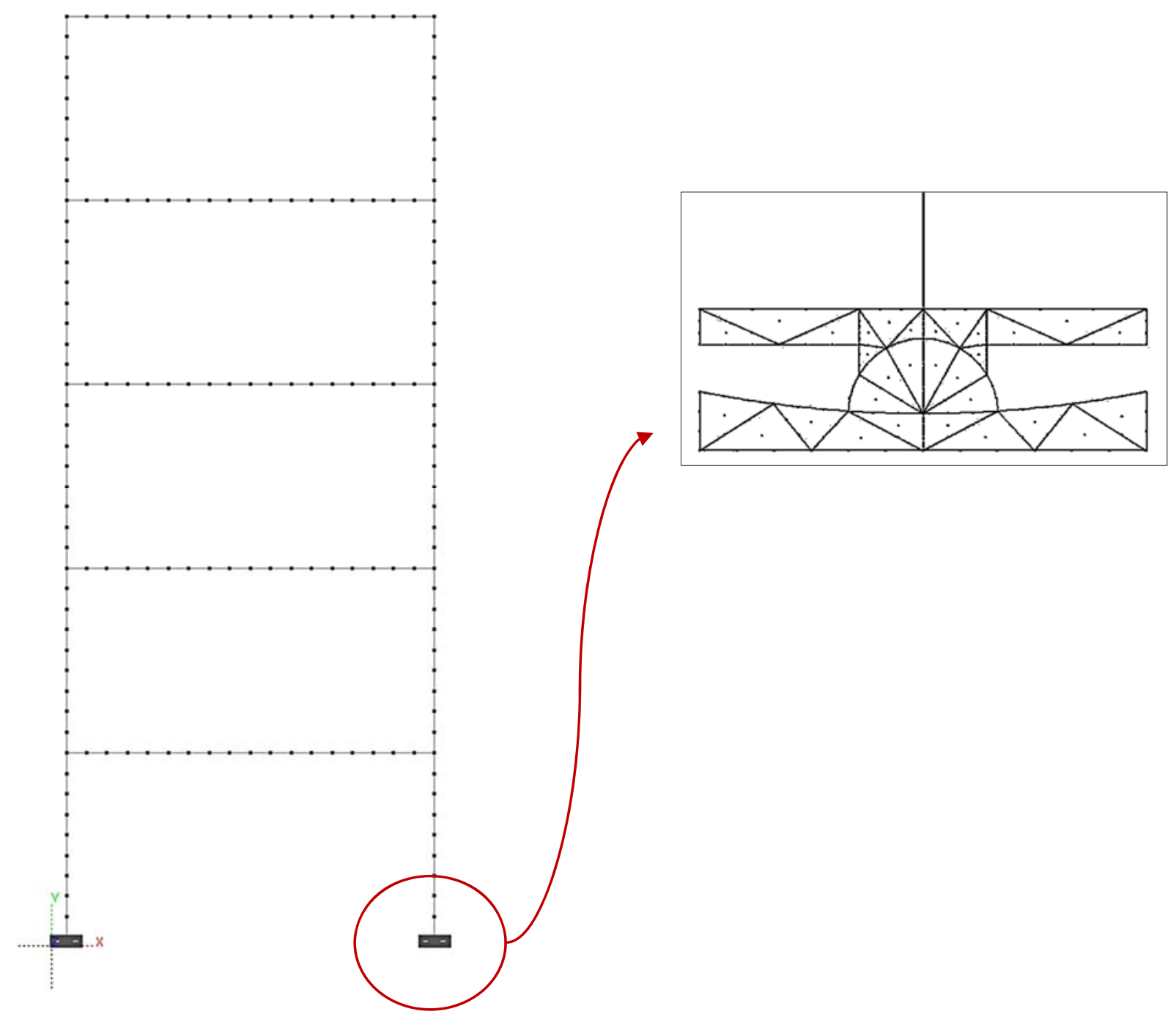

Figura 44 - Malha de elementos finitos para análise da estrutura isolada com dispositivos FPS. 
4.2.1 Caso a. Estrutura isolada na base com dispositivos FPS submetida às componentes horizontal e vertical de um terremoto real.

Na figura 45 plota-se o gráfico dos deslocamentos, horizontal e vertical, do sismo 'Superstition Hills, 1987'.

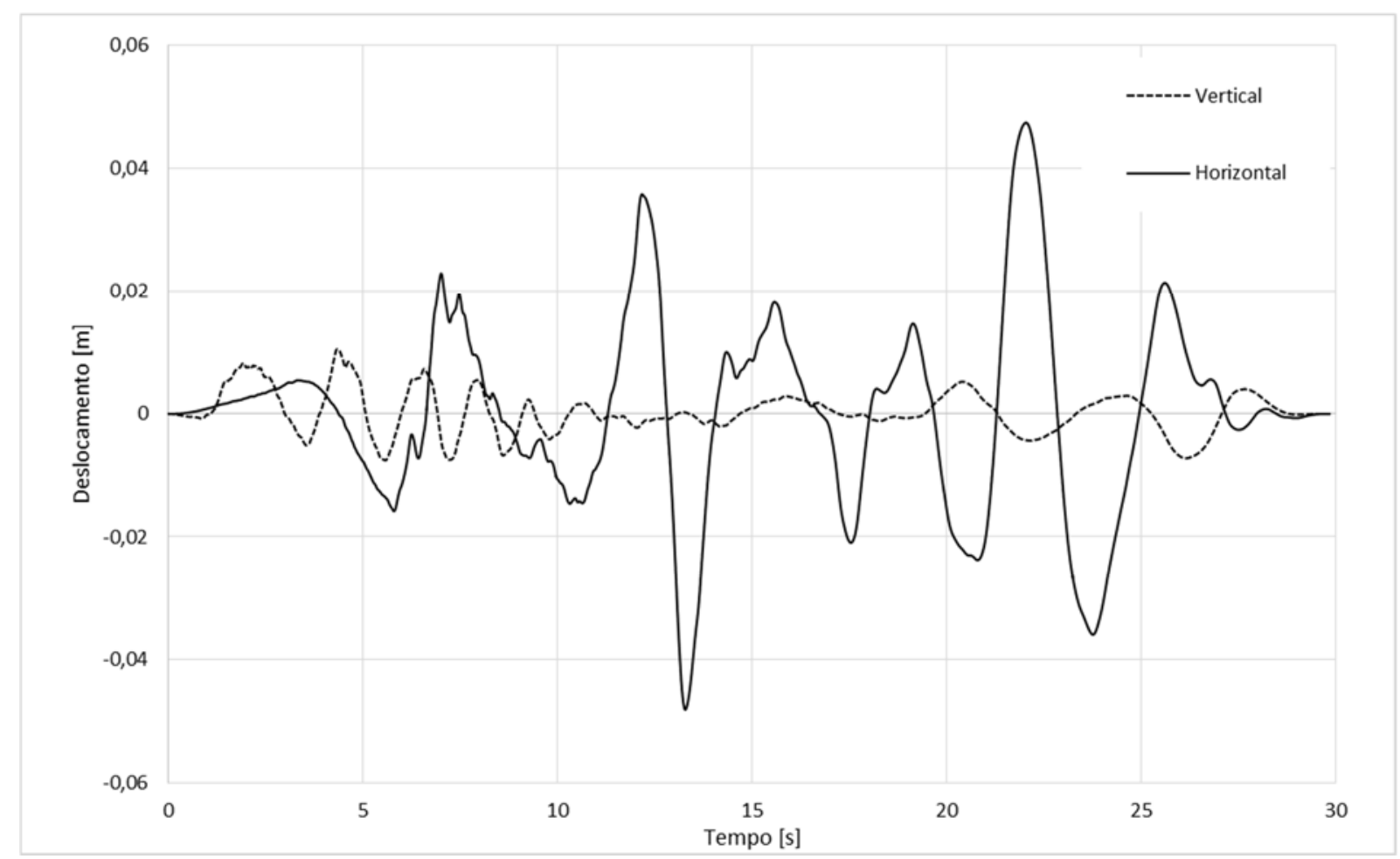

Figura 45 - Deslocamento horizontal e vertical durante o terremoto Superstition Hills, 1987.

A seguir, apresentam-se os gráficos de deslocamento e aceleração horizontal do topo da estrutura. Identificam-se os seguintes valores máximos: $17.33 \mathrm{~cm}$ e $4.926 \mathrm{~g}$, respectivamente.

Da figura 46, destaca-se o longo período de vibração da estrutura isolada, aproximadamente 2.60 segundos, quando comparado com o período de vibração mostrado na figura 38 . 


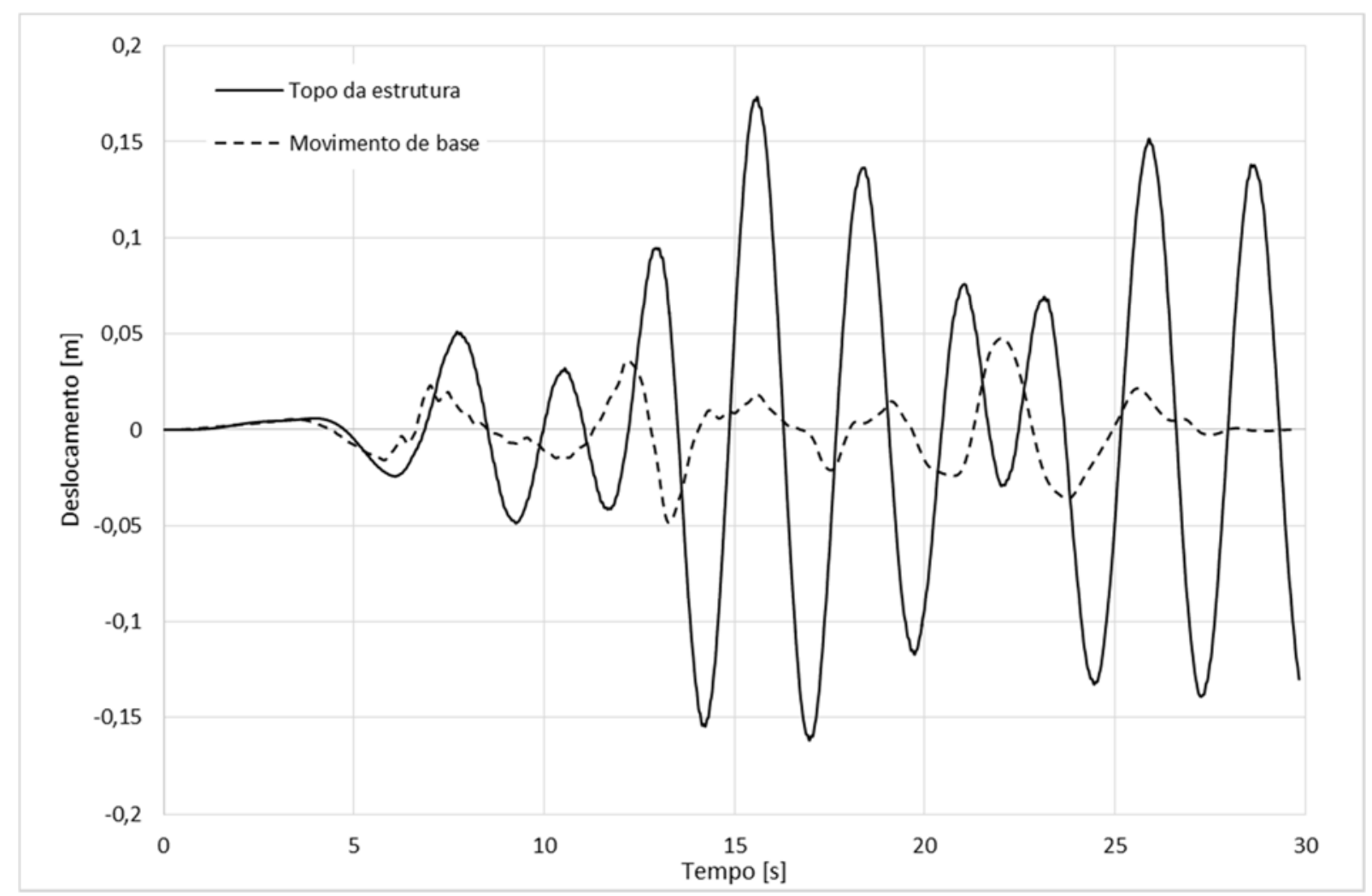

Figura 46 - Deslocamento horizontal.

A figura 47 mostra que as acelerações aumentam a medida que o tempo da análise avança, isto deve-se à desconsideração das fontes de amortecimento no problema estudado.

A figura 48 permite identificar o efeito dos dispositivos FPS na redução dos deslocamentos relativos quando comparados com a resposta estrutural do sistema convencional mostrada na figura 40. Apesar do sistema estrutural isolado com dispositivos FPS apresenta um máximo deslocamento absoluto maior do que o sistema convencional, nota-se que o controle de vibração exerce-se na redução dos deslocamentos relativos que finalmente se traduzem em uma minimização da demanda de deformação e força nos elementos da estrutura primaria.

Para ilustrar ainda mais o controle de vibração exercido, nas figuras 50, 51, 52 e 53 observa-se graficamente que a configuração deslocada da estrutura isolada encontra-se próxima à apresentada por um corpo rígido. 


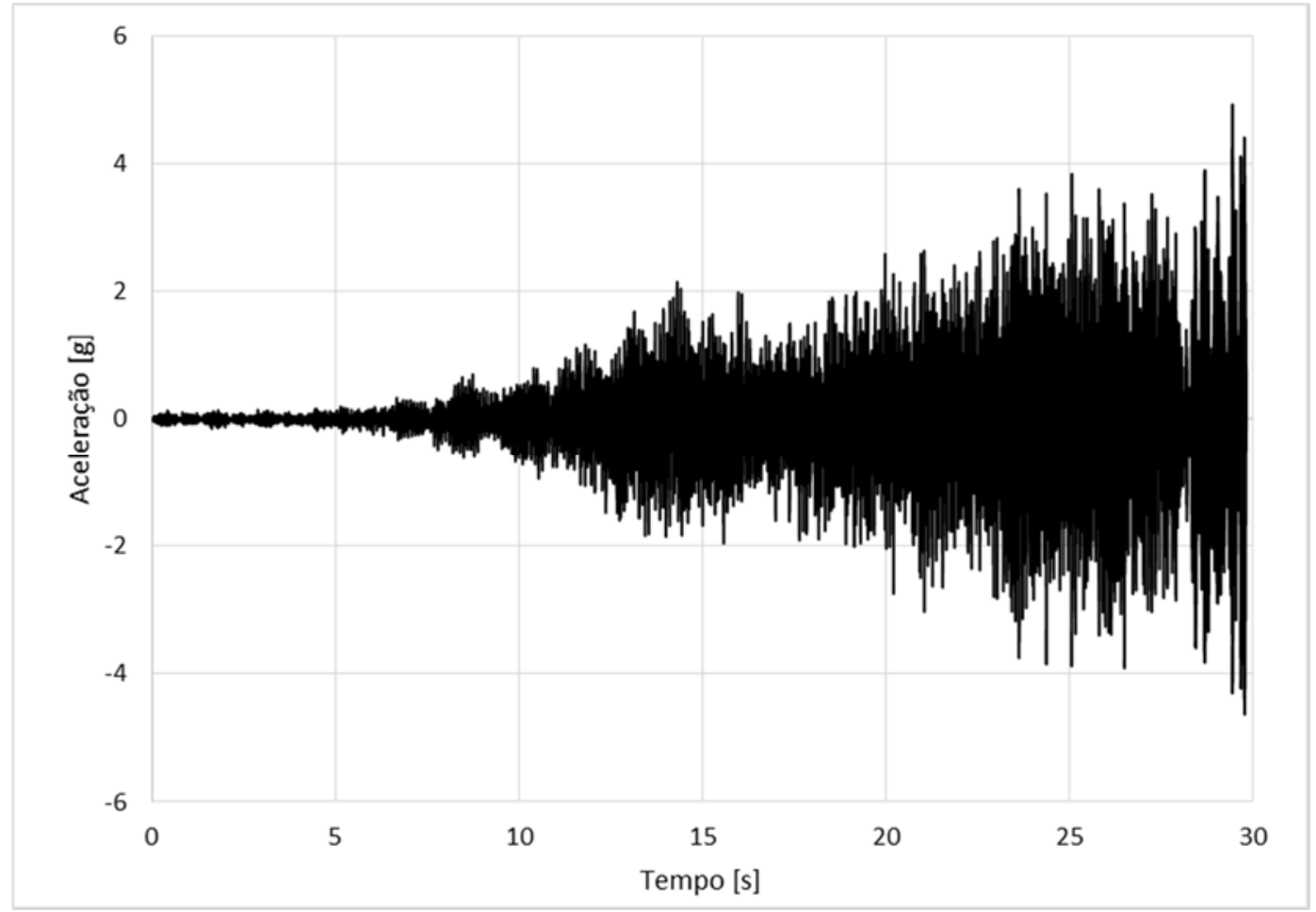

Figura 47 - Aceleração horizontal do topo da estrutura.

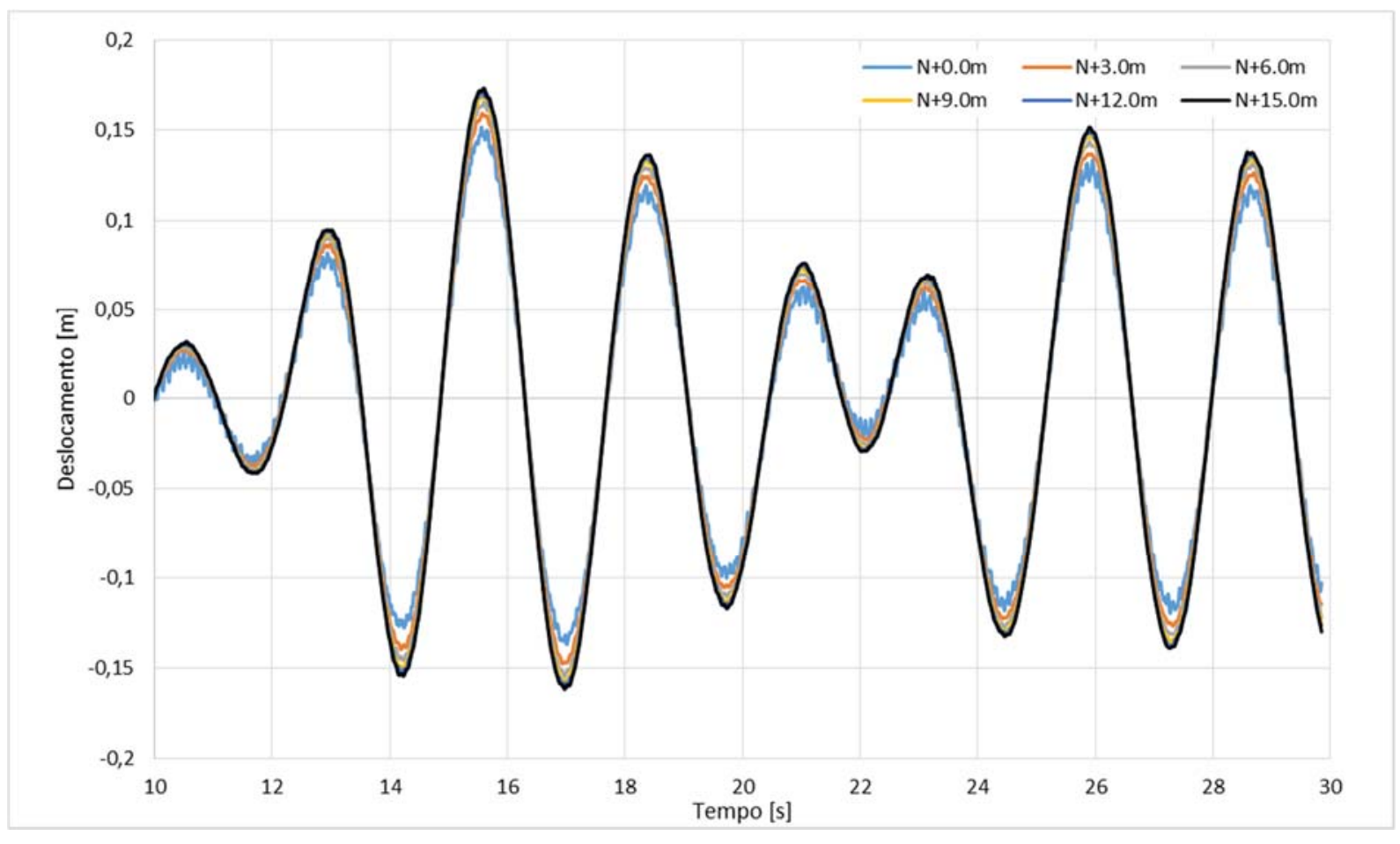

Figura 48 - Deslocamento horizontal do pilar do eixo esquerdo. 
A seguinte figura plota a força de contato obtida nos 7 nós que definem a ligação deslizante entre o deslizador articulado e a superfície côncava do isolador esquerdo para diferentes instantes de tempo.

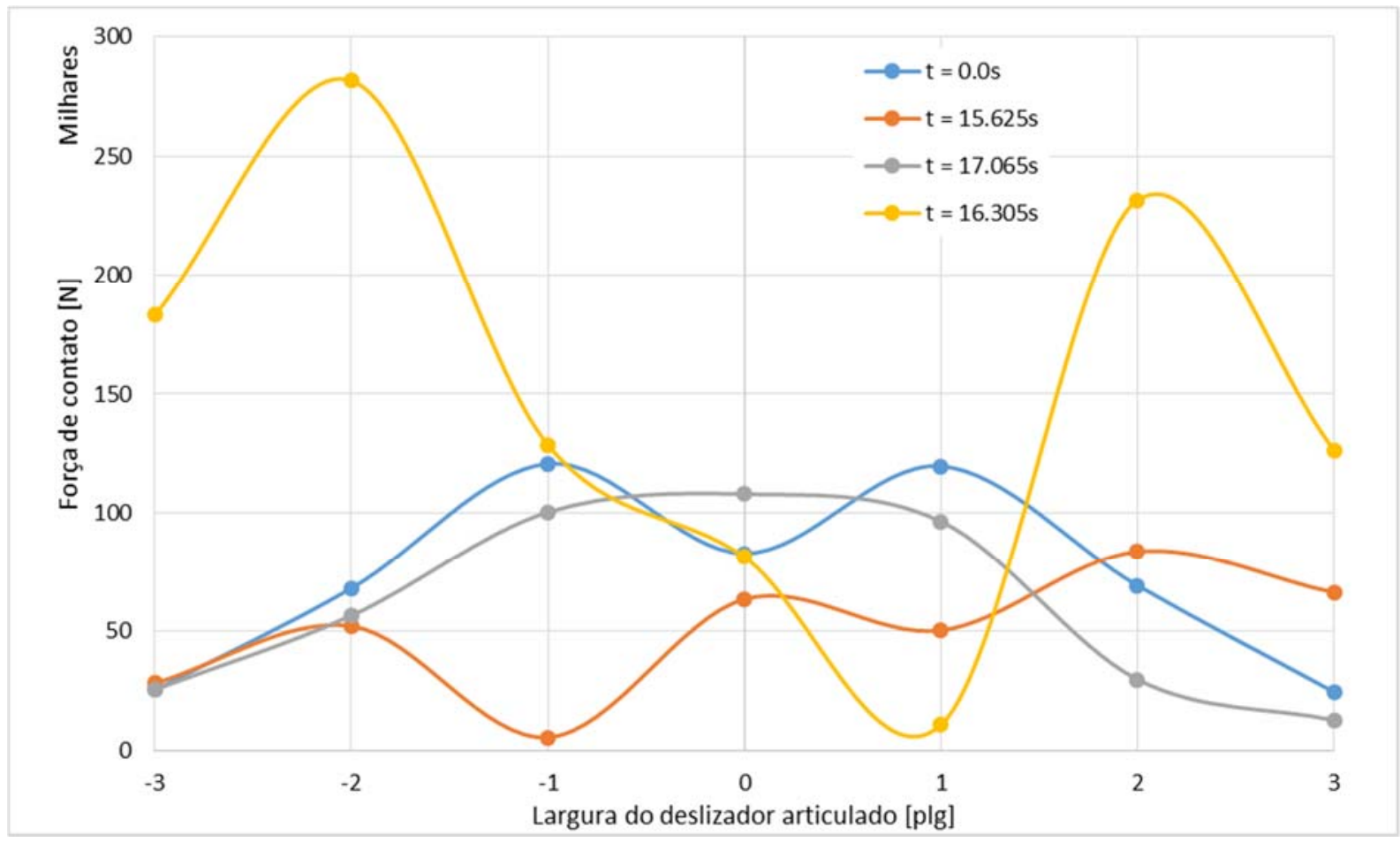

Figura 49 - Força de contato entre o deslizador articulado e a superfície côncava no FPS esquerdo.

(a) Somente carregamento estático ( $\mathrm{t}=0.0 \mathrm{~s})$. (b) Instante do máximo deslocamento horizontal 15.625 segundos (c) Instante do mínimo deslocamento horizontal 17.065 segundos (d) Instante de deslocamento horizontal nulo 16.305 segundos. 

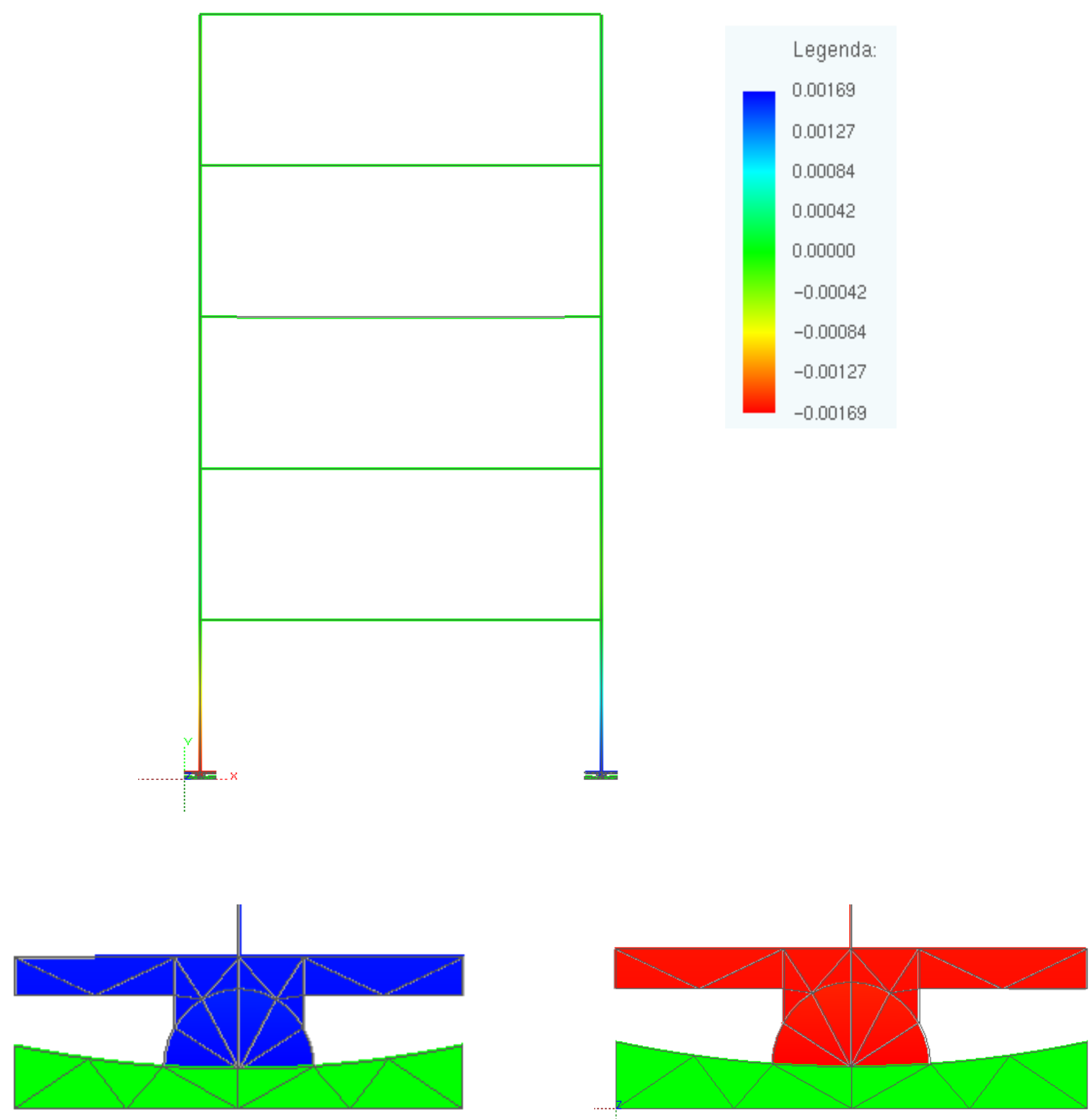

Figura 50 - Deformada após a aplicação do carregamento estático $(t=0.0 \mathrm{~s})$. (a) Isolador esquerdo (b). Isolador direito. 


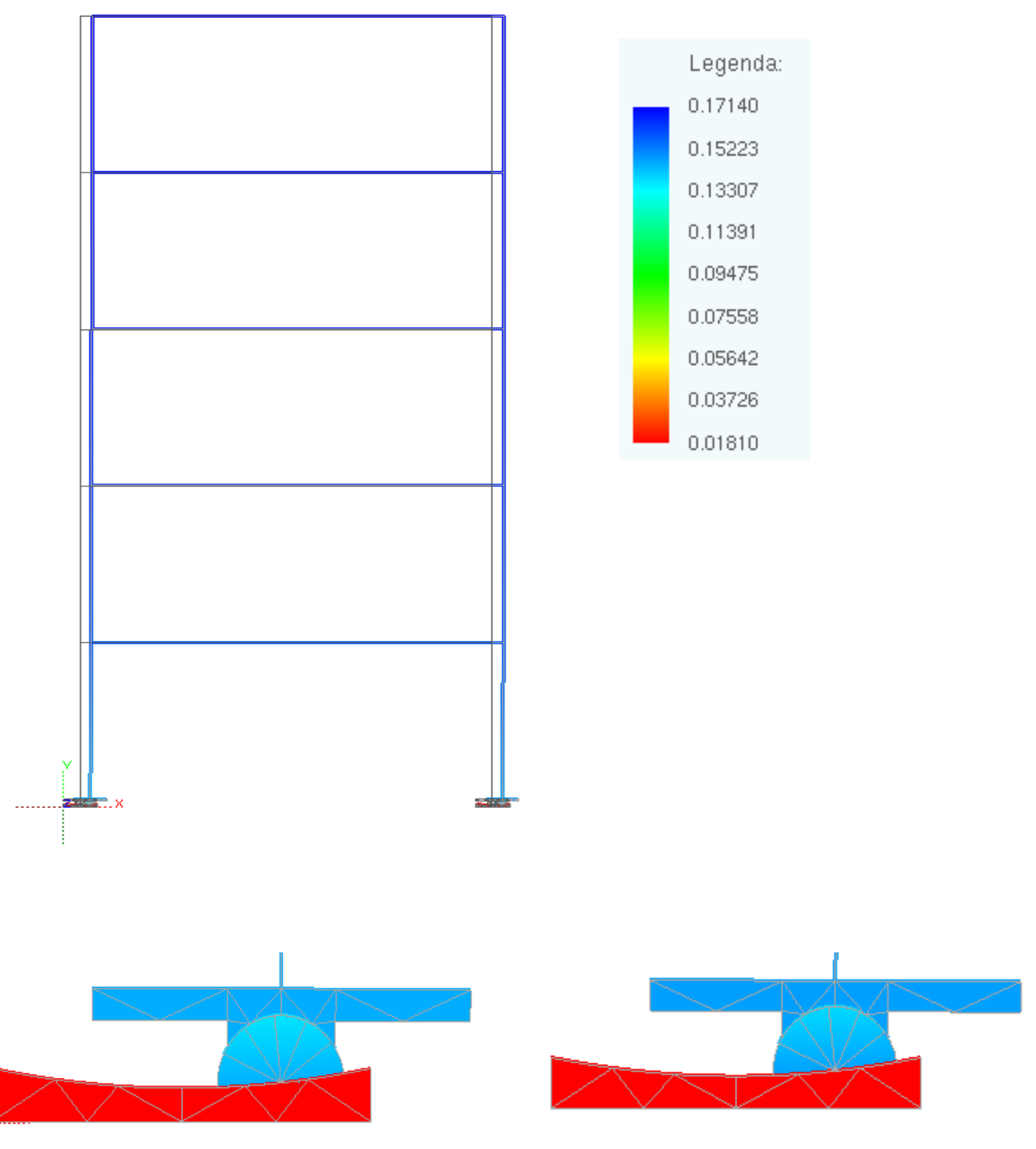

Figura 51 - Deformada instante $t=15.625 \mathrm{~s}(\mathrm{a})$ Isolador esquerdo (b) Isolador direito. 

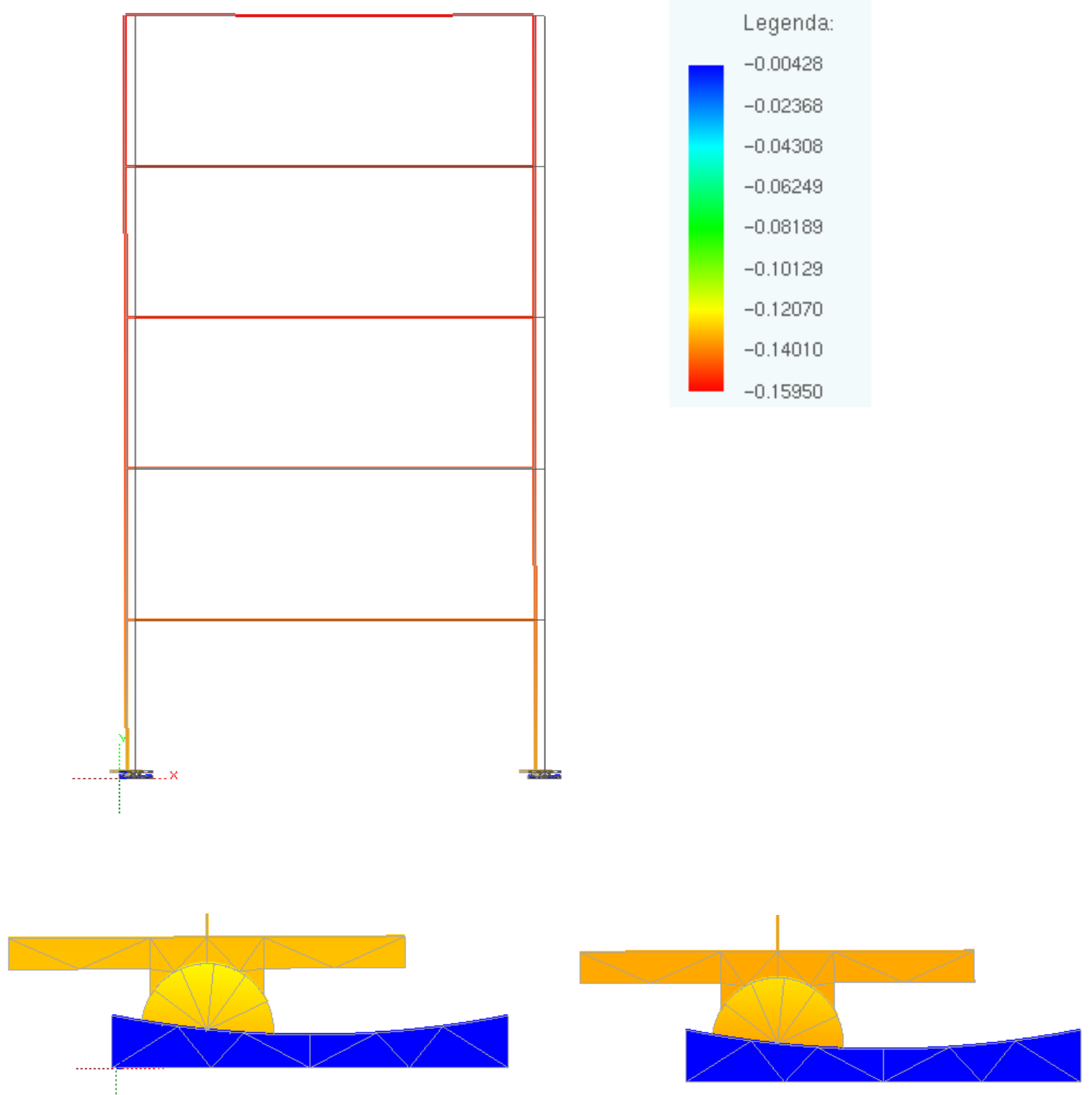

Figura 52 - Deformada instante $t=17.065 \mathrm{~s}(\mathrm{a})$ Isolador esquerdo (b) Isolador direito. 

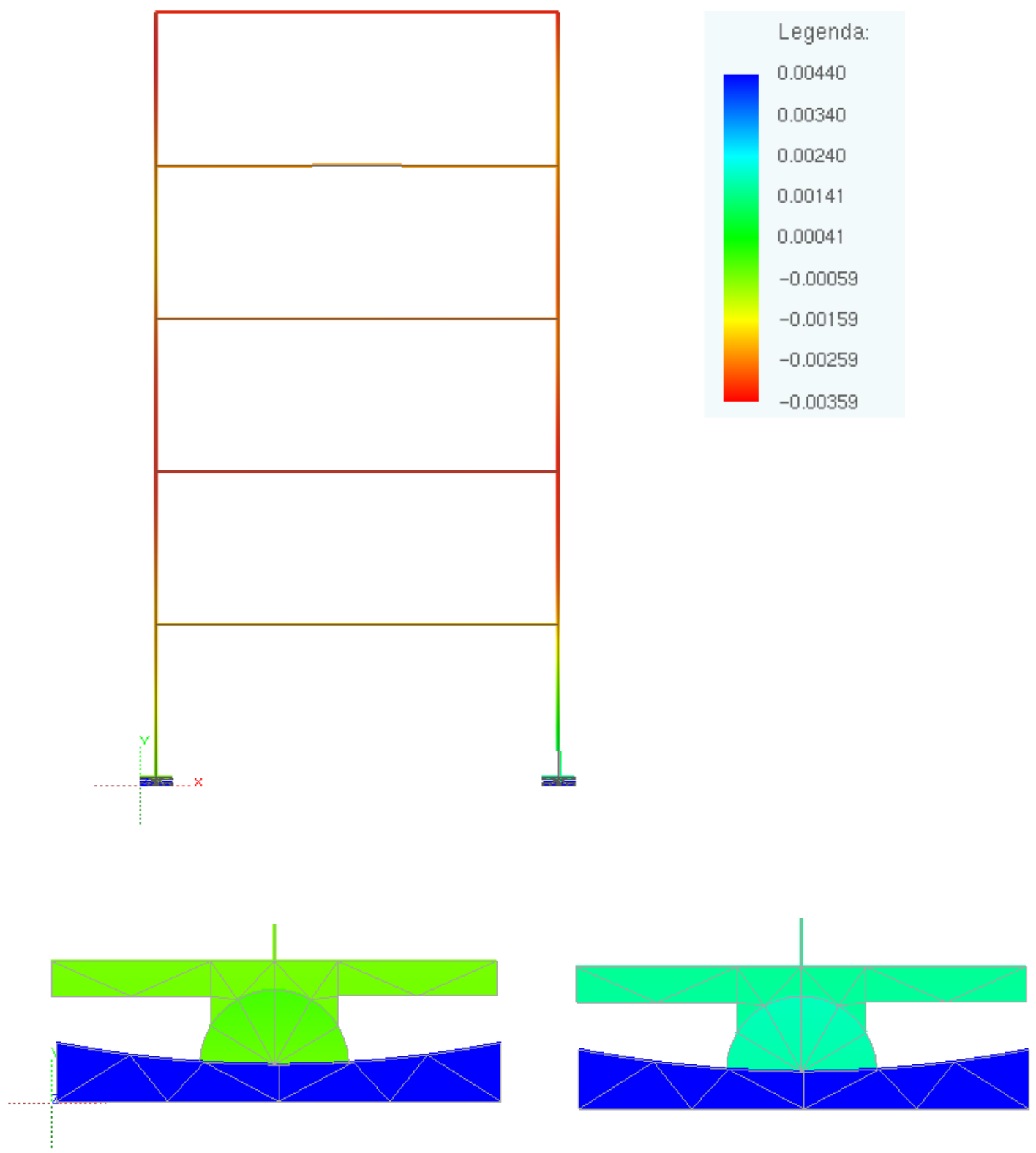

Figura 53 - Deformada instante $t=16.305 \mathrm{~s}(\mathrm{a})$ Isolador esquerdo (b) Isolador direito. 


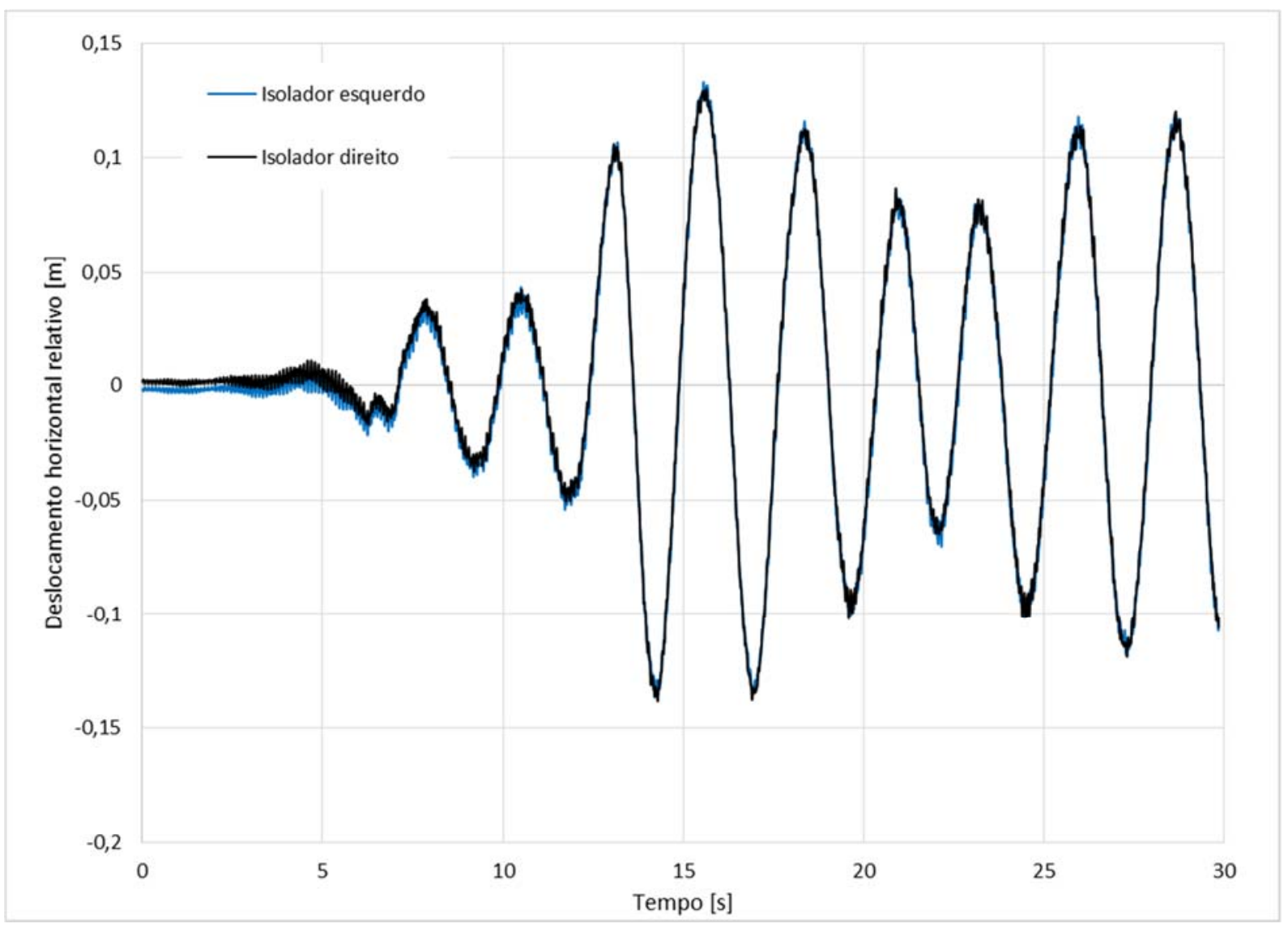

Figura 54 - Deslocamento horizontal relativo medido entre o centro do contorno da superfície côncava e o centro do contorno do deslizador articulado. 
4.2.2 Caso b. Estrutura isolada na base com dispositivos FPS submetida à componente horizontal de um terremoto real.

Nesse item aplicou-se somente a componente horizontal do terremoto com o propósito de comparar a resposta obtida, em termos de deslocamento e forças de contato, com a resposta estrutural quando considerada também a componente vertical, como feito no item 4.2.1.

Nota-se que a evolução do deslocamento horizontal do topo da estrutura (ver figura 55) fica superposta à resposta encontrada ao considerar as duas componentes do terremoto.

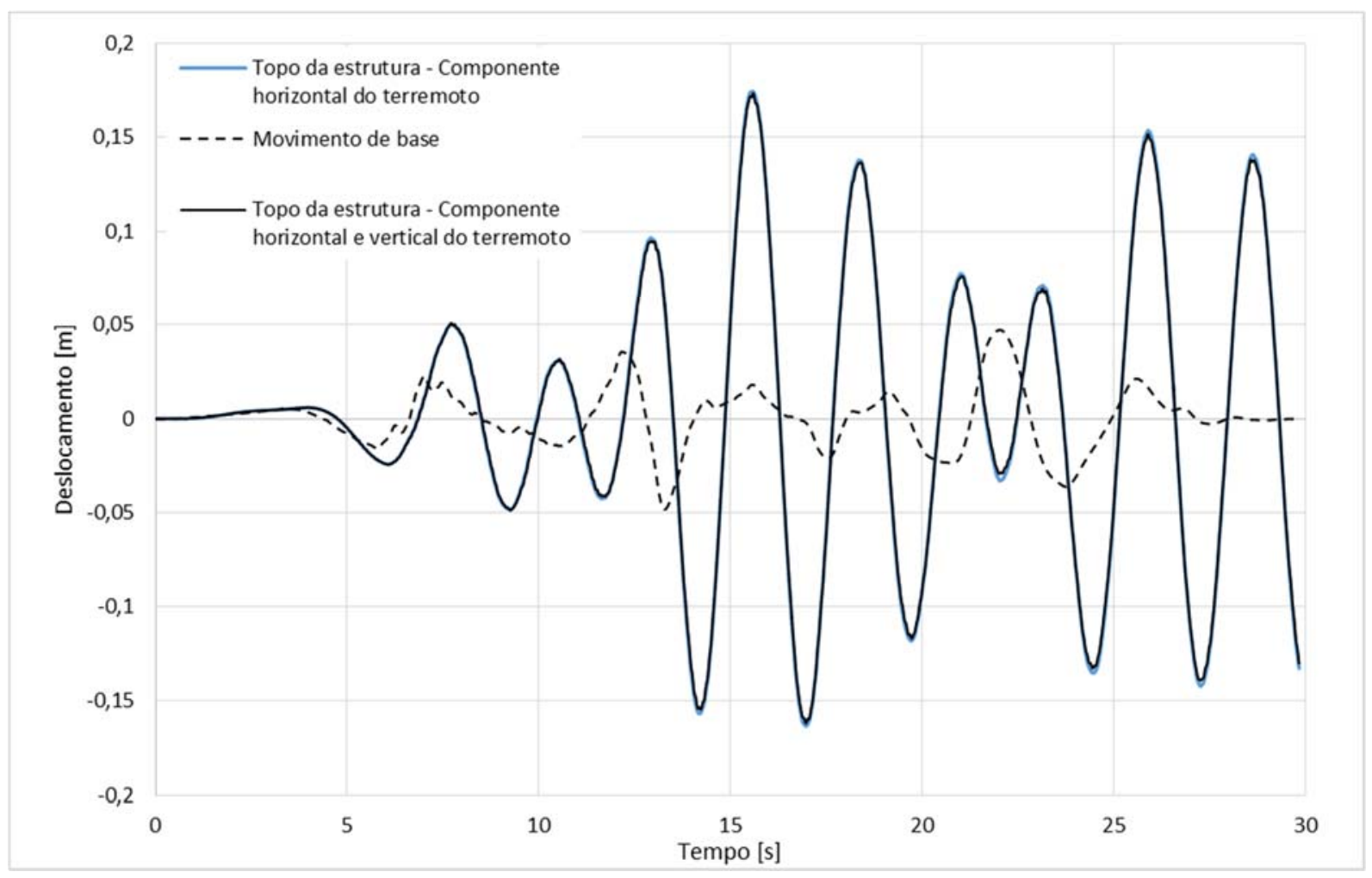

Figura 55 - Deslocamento horizontal.

No entanto, ao comparar as forças de contato desenvolvidas no FPS esquerdo para os mesmos instantes de tempo selecionados no item anterior, calculam-se diferenças que oscilam entre 1.0\% e 70\% (Ver figuras 49 e 56). 


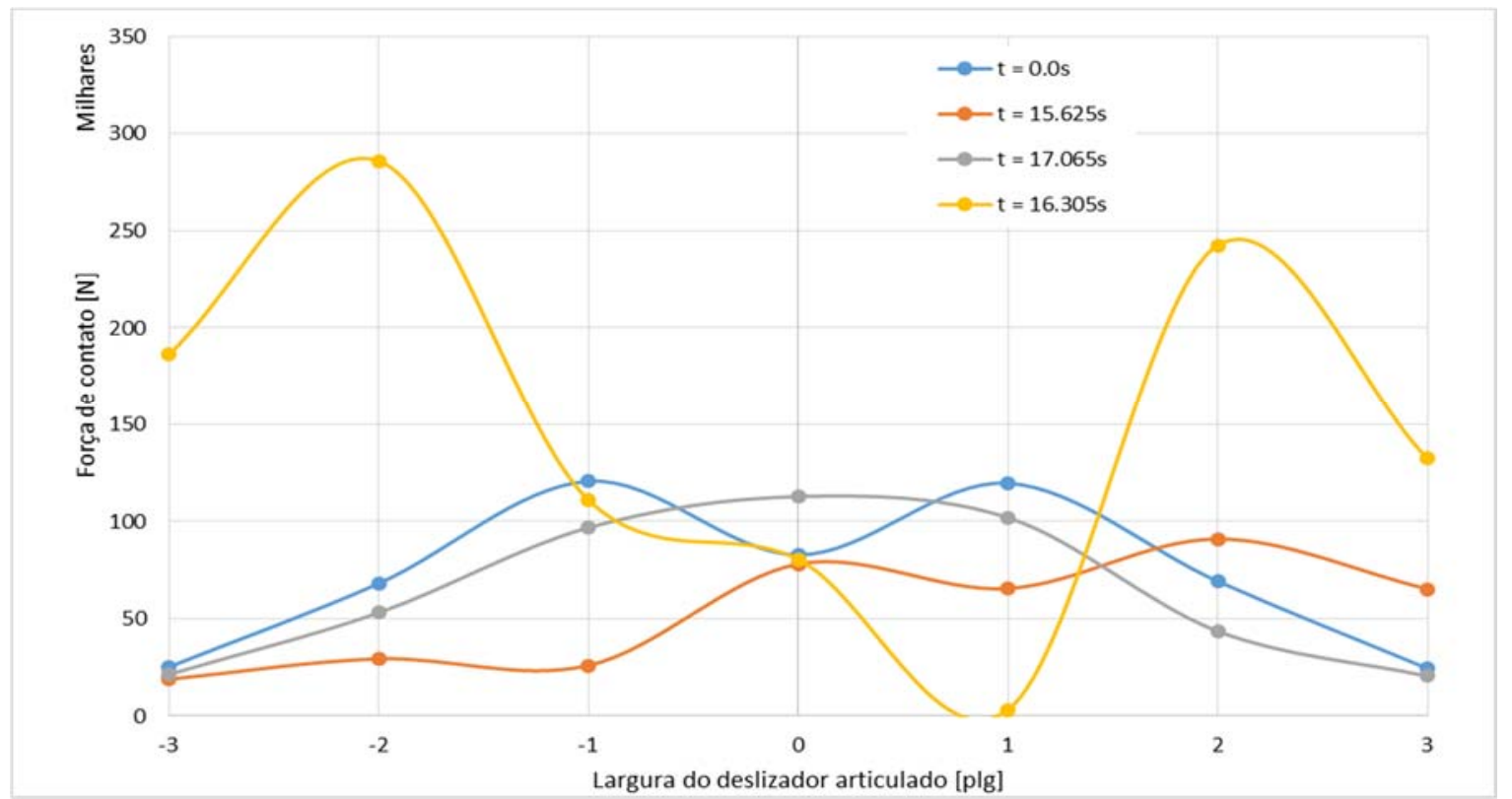

Figura 56 - Força de contato entre o deslizador articulado e a superfície côncava no FPS esquerdo.

(a) Somente carregamento estático ( $\mathrm{t}=0.0 \mathrm{~s})$. (b) Instante do máximo deslocamento horizontal 15.625 segundos (c) Instante do mínimo deslocamento horizontal 17.065 segundos (d) Instante de deslocamento horizontal nulo 16.305 segundos. 


\subsection{Estrutura com sistema TMD.}

No presente item apresenta-se a modelagem e os resultados obtidos da análise de uma estrutura com o sistema de controle TMD empregando o dispositivo massa/mola/amortecedor descrito no capítulo 2.

A figura 57 mostra o modelo de elementos finitos utilizado. No topo da estrutura, instalou-se o dispositivo massa/mola/amortecedor e atribuíram-se as seguintes propriedades geométricas e físicas: área $250 \mathrm{~cm}^{2}$, comprimento inicial 3.0m, densidade nula, modulo de elasticidade 10MPa, viscosidade dinâmica 4MPa-s e uma massa concentrada de 13 toneladas. As propriedades da estrutura primaria mantiveram-se iguais às empregadas nos itens 4.2 e 4.1.

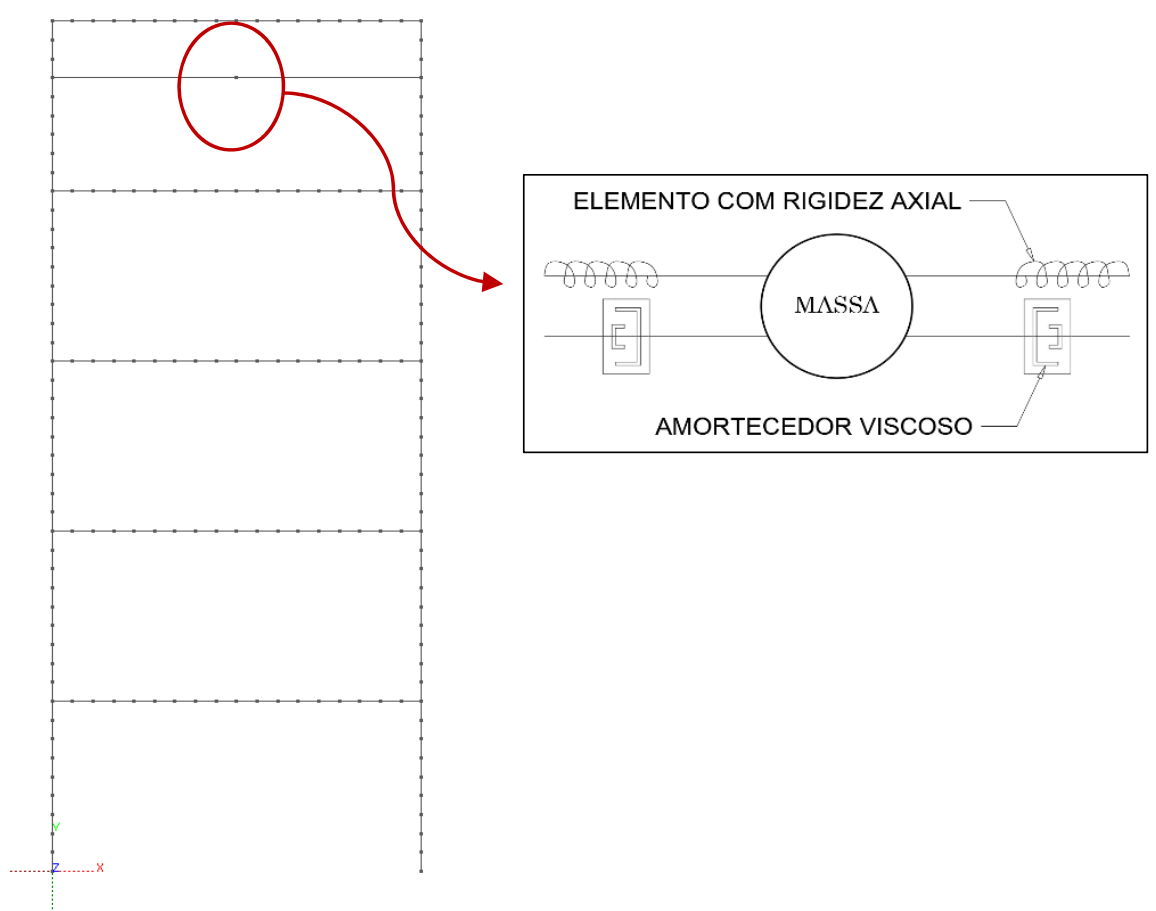

Figura 57 - Detalhe do modelo de elementos finitos da estrutura com o sistema TMD.

Os resultados obtidos em termos de deslocamento horizontal plotam-se na figura 58. Observa-se a capacidade da formulação na representação do fenômeno de absorção de vibração por parte do dispositivo TMD. O deslocamento absoluto do topo da estrutura é substancialmente reduzido, aproximadamente $40 \%$, em relação 
ao deslocamento apresentado pela estrutura sem nenhum tipo de controle de vibração.

É importante comentar que o valor escolhido para a massa concentrada do TMD, aproximadamente $10 \%$ da massa da estrutura primaria, não resultou de uma estratégia de otimização dado que foge do objetivo do presente trabalho. Simplesmente, tomando como referência a sugestão de alguns autores de utilizar relações de massa que oscilem entre o $1 \%$ e o $20 \%$ para aplicações civis (WARBURTON, 1982) (SOONG; DARGUSH, 1997), o valor definiu-se arbitrariamente. Mesmo assim, o exemplo evidencia a eficiência do TMD no controle de vibração de estruturas.

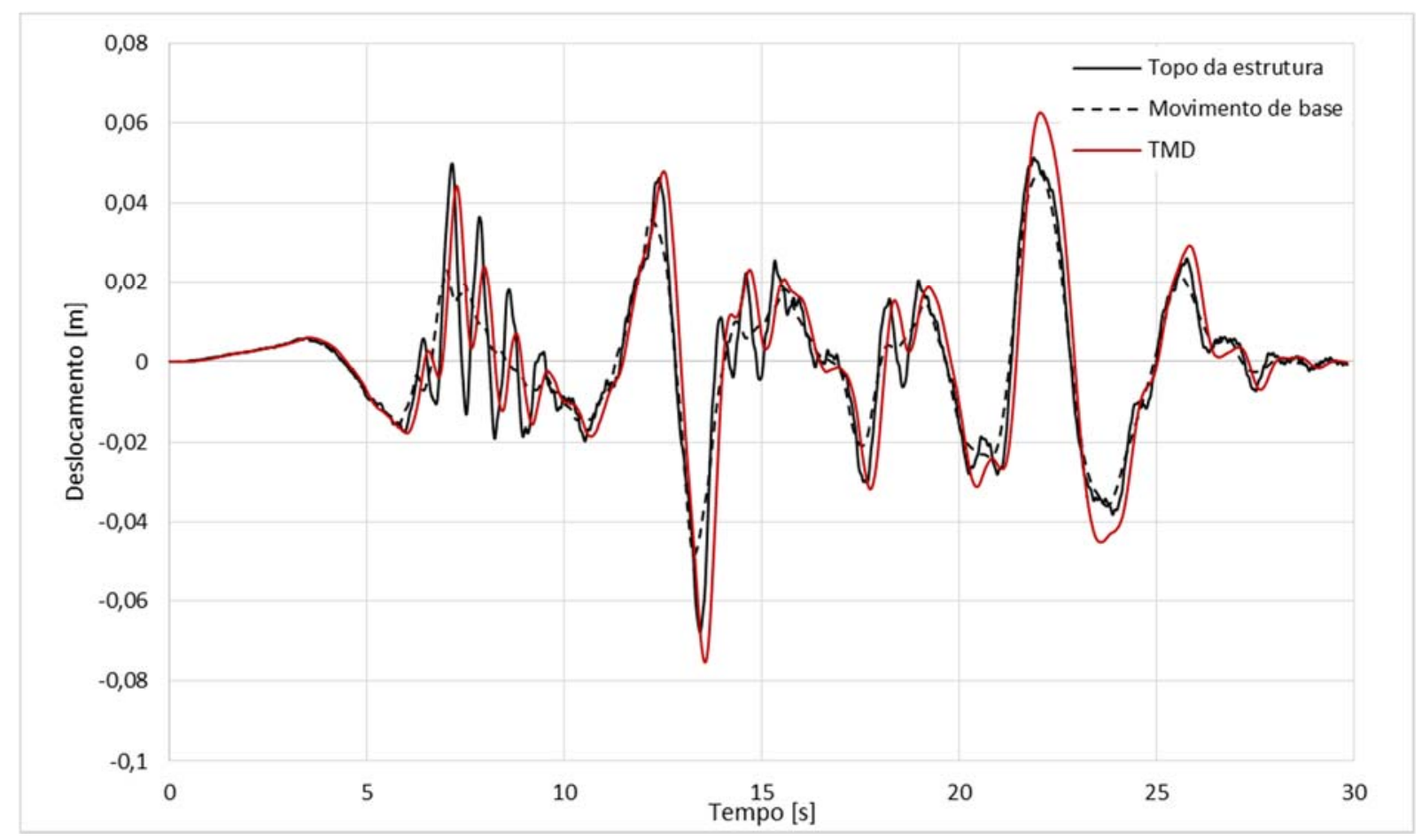

Figura 58 - Deslocamento horizontal.

A figura 60 e 61 apresentam a aceleração horizontal do topo da estrutura e da massa do TMD, respectivamente. O sistema de controle reduziu $37 \%$ a aceleração máxima do topo da estrutura. Acelerações maiores apresentaram-se no dispositivo TMD. 


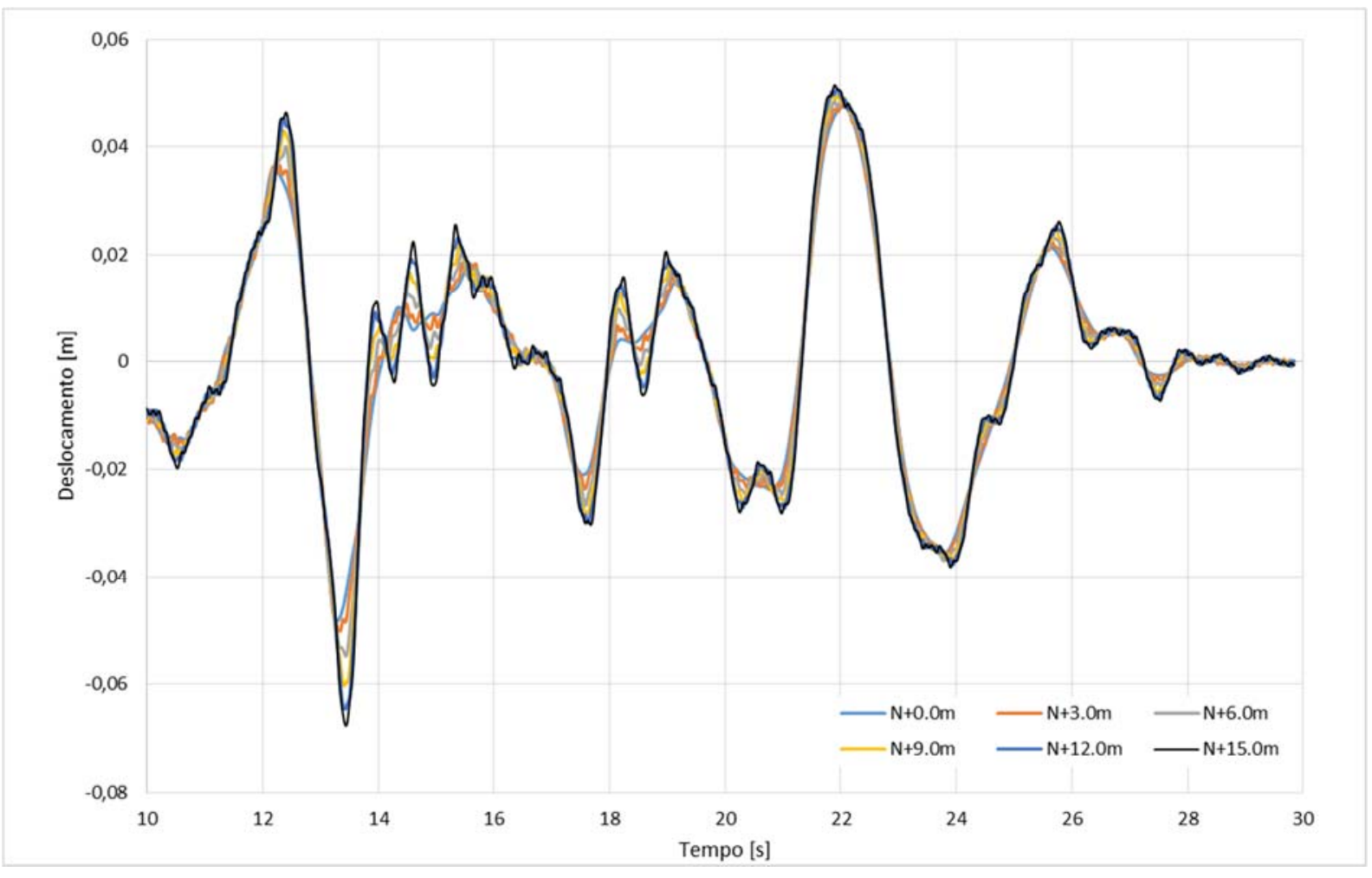

Figura 59 - Deslocamento horizontal do pilar do eixo esquerdo.

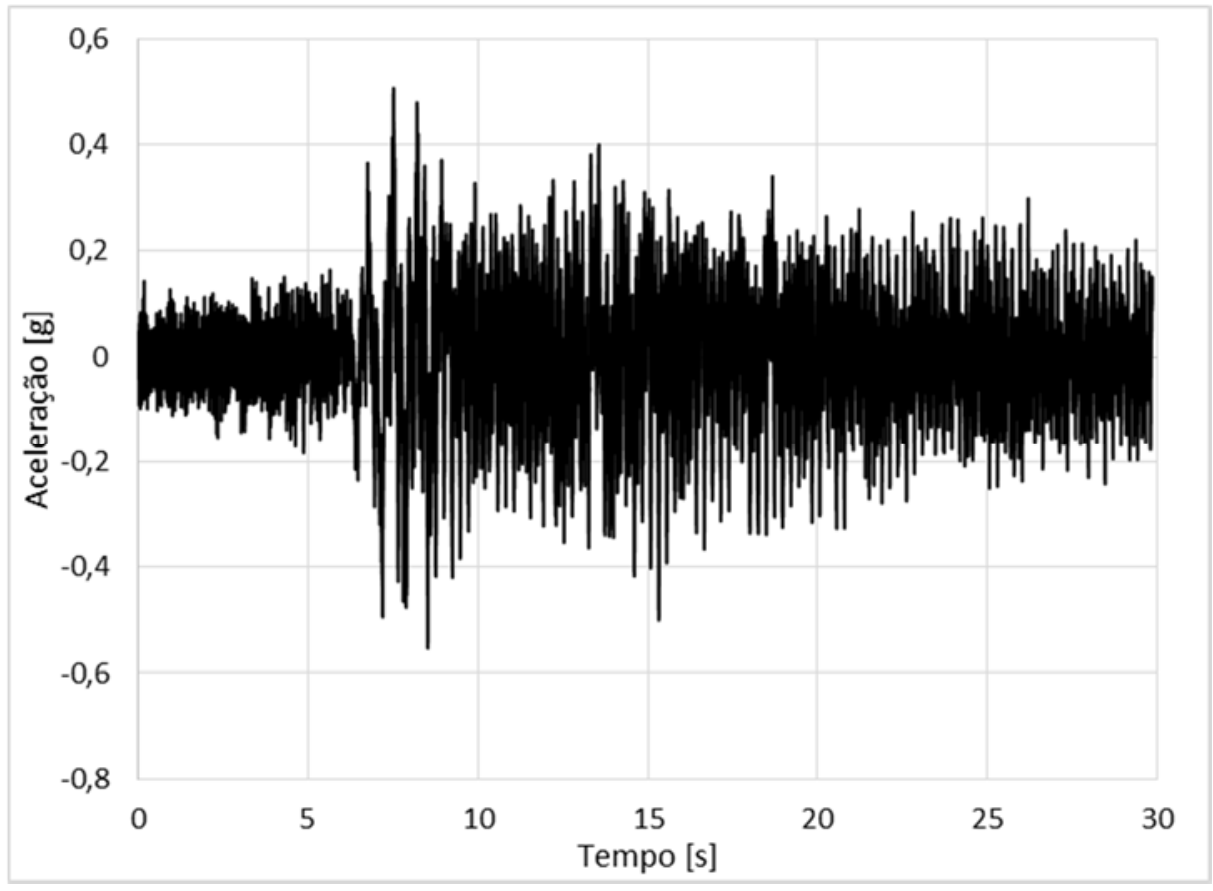

Figura 60 - Aceleração horizontal do topo da estrutura. 


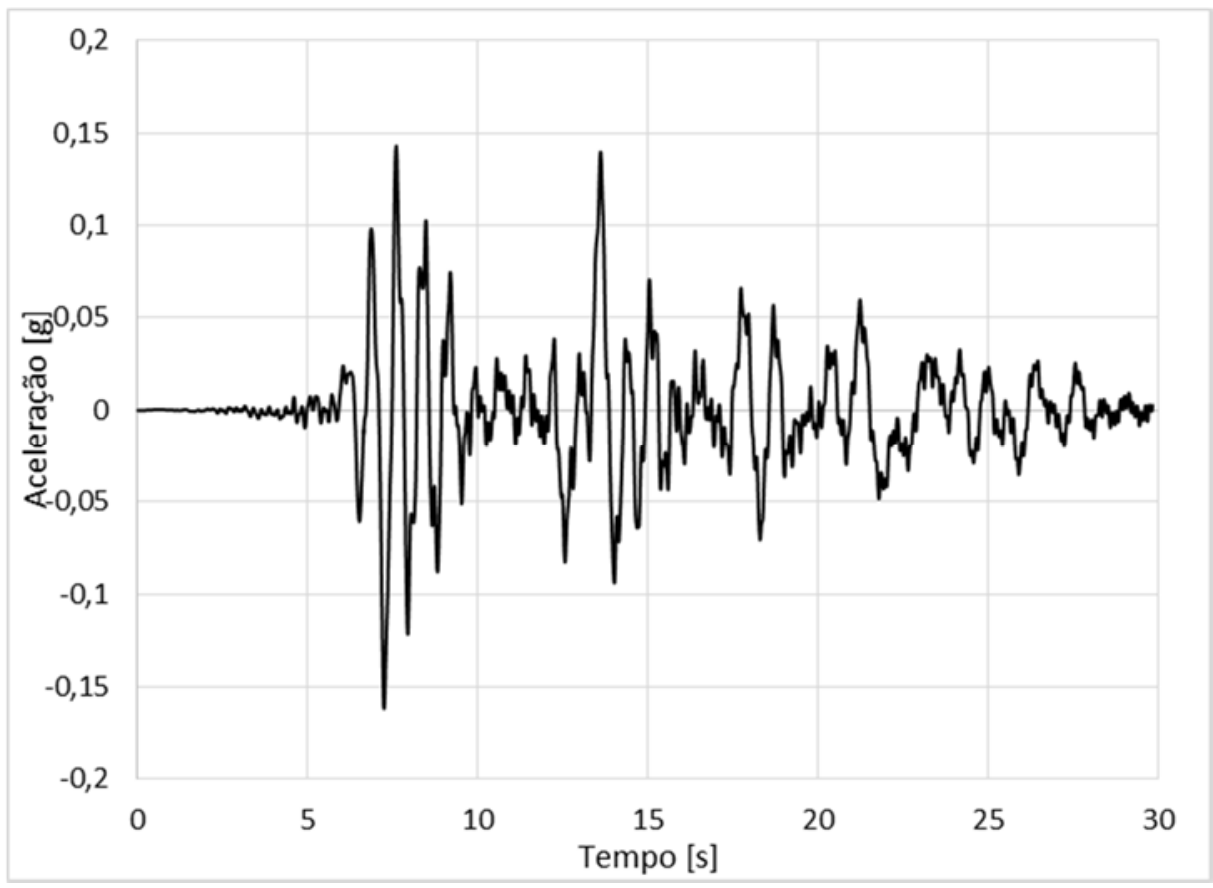

Figura 61 - Aceleração horizontal do TMD.

4.4 Estrutura com sistema TMD e isolada com dispositivos FPS.

No presente item combinam-se os dois sistemas de controle de vibração empregando as estratégias de modelagem descritas anteriormente. Mantiveram-se ainda as propriedades geométricas e físicas definidas tanto para a estrutura primaria quanto para os dispositivos de controle.

A figura 62 mostra a geometria do modelo de elementos finitos analisado.

Os deslocamentos horizontais obtidos plotam-se na figura 63. O efeito de absorção do TMD evidencia-se na redução da amplitude de movimento do topo da estrutura quando comparado com a figura 46 . Passou de $17.33 \mathrm{~cm}$ a $13.37 \mathrm{~cm}$ de deslocamento máximo horizontal. 


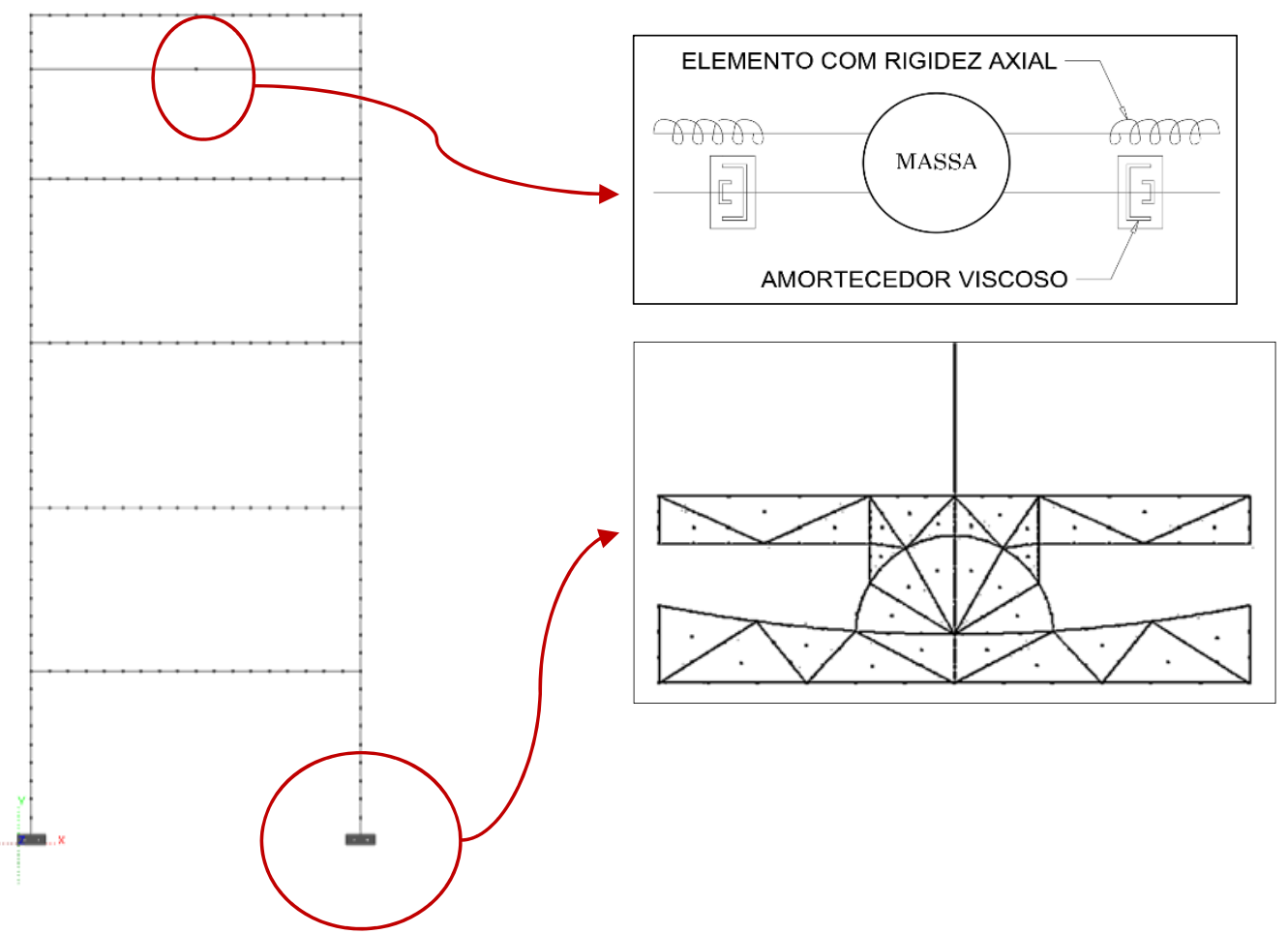

Figura 62 - Detalhe do modelo de elementos finitos.

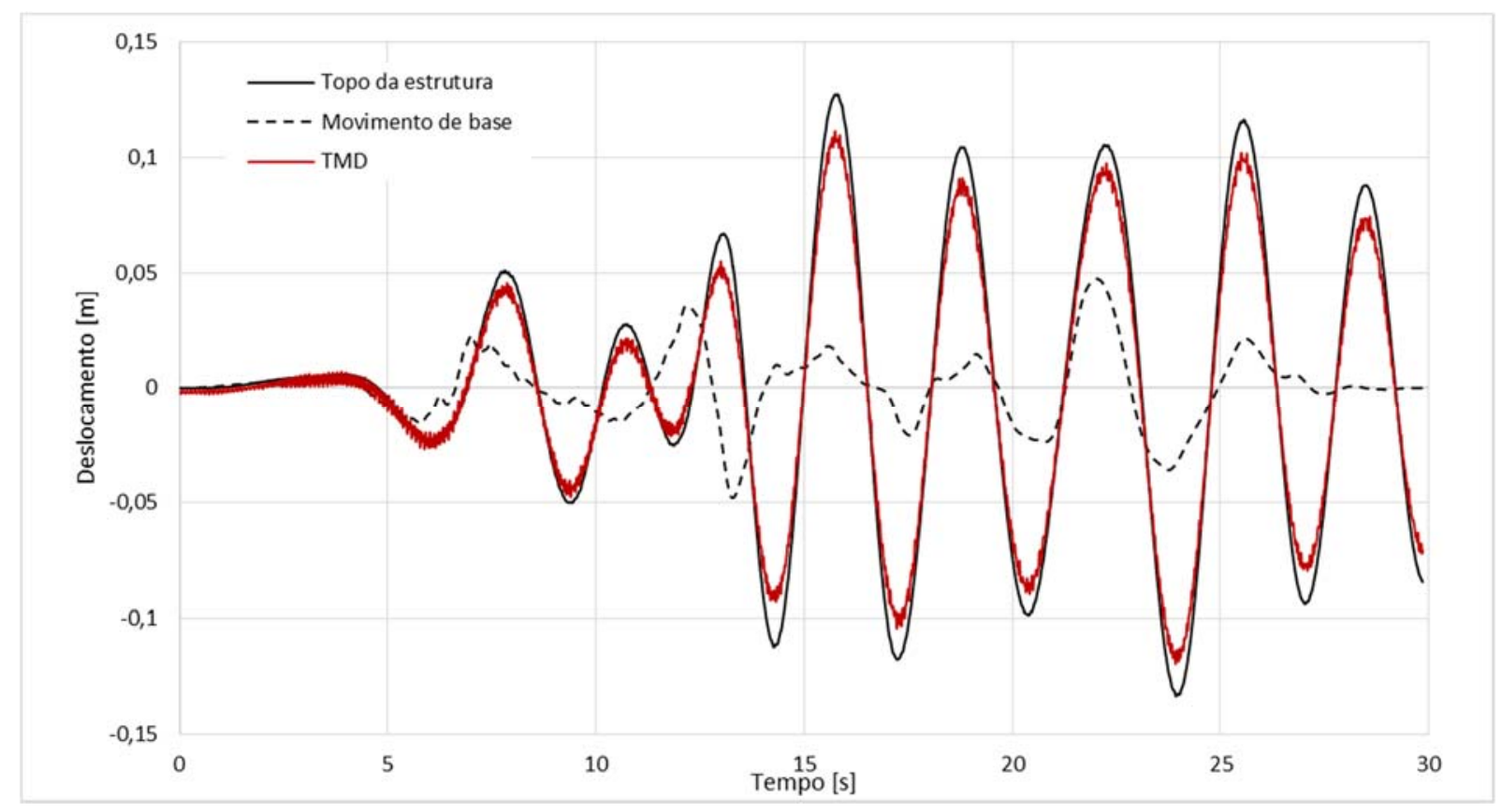

Figura 63 - Deslocamento horizontal.

Comparando o período de vibração obtido da figura $63 \mathrm{com}$ o período de vibração obtido da figura 55 nota-se uma diferencia apesar de ter utilizado o mesmo raio de curvatura do dispositivo FPS. Essa diferencia deve-se a que na análise 
descrita nesse item somente foi considerada a massa concentrada do TMD para efeitos dinâmicos e não foi considerado o seu aporte no peso próprio da estrutura. 


\section{CONCLUSÕES}

Neste trabalho implementou-se a formulação posicional do método dos elementos finitos para análise não linear geométrica estática e dinâmica de estruturas planas em conjunto com a técnica dos multiplicadores de Lagrange para a imposição de restrições cinemáticas. Propôs-se uma estratégia para resolver o problema de contato deslizante liso entre elementos sólidos bidimensionais.

Abordou-se o deslizamento entre elementos sólidos bidimensionais como um conjunto de ligações cilíndricas constituído pelos nós dos elementos deslizantes e pelas superfícies mestres definidas por linhas parametrizadas de forma análoga a elementos de pórtico. Cada nó, condicionado à geometria da superfície deslizante, representa uma parcela adicional no funcional de energia total do sistema, cuja incógnita é o multiplicador de Lagrange. A formulação implementada para resolver o deslizamento caracteriza-se por utilizar a coordenada curvilínea associada ao comprimento real da trajetória como grau de liberdade, dando significado físico às grandezas relacionadas à força e massa do nó conectado.

Empregaram-se elementos finitos, tanto de barra geral quanto de chapa, isoparamétricos de aproximação cúbica que permitem capturar facilmente geometrias curvas, aspecto preponderante na modelagem dos dispositivos do tipo FPS de isolamento de estruturas modelados.

Aplicou-se o método incremental-iterativo de Newton Raphson, escrito na sua forma clássica, para a resolução do sistema não linear de equações e o método de Newmark da aceleração média como integrador temporal. Todas as parcelas que participam no algoritmo de solução foram apresentadas e escritas de forma a facilitar sua implementação computacional. Foram introduzidas as equações de restrição via Multiplicadores de Lagrange no método de solução.

Analisaram-se vários exemplos dinâmicos no âmbito da simulação numérica de sistemas de controle de vibração de estruturas, mostrando a capacidade da formulação de representar os fenômenos de isolação e absorção, especialmente. Destaca-se a estabilidade numérica que apresentou a ferramenta computacional mesmo quando várias restrições cinemáticas foram impostas no problema estudado. 
Conclui-se, finalmente, que a formulação proposta foi capaz de modelar com precisão os dispositivos de controle de vibração abordados e que o programa resultante pode ser usado como uma ferramenta prática para a análise dinâmica não linear de estruturas e mecanismos. 


\subsection{Trabalhos futuros}

Ampliar as aplicações da formulação implementada analisando outros dispositivos de controle de vibração como os sistemas de deslizamento duplo, triplo e de frequência variável para isolamento de estruturas e sistemas a base de amortecedores viscosos. Inclusive estudar mecanismos usados nas industrias da engenharia mecânica, aeronáutica, aeroespacial e armamentista.

Estender a formulação para a modelagem de estruturas tridimensionais e incluir atrito seco nas ligações deslizantes para análises estáticas e dinâmicas. 


\section{REFERENCIAS}

ALMAZÁN, J. L.; DE LA LLERA, J. C.; INAUDI, J. A. Modelling aspects of structures isolated with the frictional pendulum system. Earthquake Engineering and Structural Dynamics, v. 27, n. 8, p. 845-867, 1998.

ALMAZÁN, J. L.; DE LA LLERA, J. C. Analytical model of structures with frictional pendulum isolators. Earthquake Engineering \& Structural Dynamics, v. 31, n. 2, p. 305, 2002.

BAUCHAU, O. A. On the modeling of prismatic joints in flexible multi-body systems. Computer Methods in Applied Mechanics and Engineering, v. 181, n. 1-3, p. 87-105, 2000.

BAUCHAU, O. A.; BOTTASSO, C. L. Contact Conditions for Cylindrical, Prismatic, and Screw Joints in Flexible Multibody Systems. Multibody System Dynamics, v. 5, n. 3, p. 251-278, 2001. Kluwer Academic Publishers.

BEHDINAN, K.; STYLIANOU, M. C.; TABARROK, B. Co-rotational dynamic analysis of flexible beams. Computer Methods in Applied Mechanics and Engineering, v. 154, n. 3-4, p. 151-161, 1998.

BISSHOPP, K. E.; DRUCKER, D. C. Large Deflection of Cantilever Beams. Quarterly of Applied Math, v. 3, n. 3, p. 272-275, 1945.

BONDONET, G.; FILIATRAULT, A. Frictional response of PTFE sliding bearings at high frequencies. Journal of Bridge Engineering, v. 2, n. November, p. 139-148, 1997. American Society of Civil Engineers.

CARDONA, A.; GERADIN, M.; DOAN, D. B. Rigid and flexible joint modelling in multibody dynamics using finite elements. Computer Methods in Applied Mechanics and Engineering, v. 89, n. 1-3, p. 395-418, 1991. 
CLARKE, C. S. J.; BUCHANAN, R.; EFTHYMIOU, M.; SHAW, C. Structural Platform Solution For Seismic Arctic Environments-Sakhalin II Offshore Facilities. Offshore Technology Conference. Anais..., 2005. Offshore Technology Conference.

CLOUGH, R. W. The Finite Element Method in Plane Stress AnalysisNo Title. Proc. 2nd ASCE Conf. On Electronic Computation, Pittsburg, Pa. Anais..., 1960.

CODA, H. B. An exact FEM geometric non-linear analysis of frames based on position description. INTERNATIONAL CONGRESS OF MECHANICAL ENGINEERING. Anais..., 2003. São Paulo.

CODA, H. B.; GRECO, M. A simple FEM formulation for large deflection 2D frame analysis based on position description. Computer Methods in Applied Mechanics and Engineering, v. 193, n. 33-35, p. 3541-3557, 2004.

CODA, H. B.; PACCOLA, R. R. Unconstrained Finite Element for Geometrical Nonlinear Dynamics of Shells. Mathematical Problems in Engineering, v. 2009, p. 132, 2009.

CODA, H. B.; PACCOLA, R. R. A total-Lagrangian position-based FEM applied to physical and geometrical nonlinear dynamics of plane frames including semi-rigid connections and progressive collapse. Finite Elements in Analysis and Design, v. 91, p. 1-15, 2014.

CONSTANTINOU, M. C.; TSOPELAS, P.; KASALANATI, A.; WOLFF, E. D. Property modification factors seismic isolation bearings. , 1999.

CONSTANTINOU, M.; MOKHA, A.; REINHORN, A. Teflon Bearings in Base Isolation II: Modeling. Journal of Structural Engineering, v. 116, n. 2, p. 455-474, 1990.

COOK, R. D.; MALKUS, D. S.; PLESHA, M. . Concepts and Applications of Finite Element Analysis. Wiley, 2002.

COURANT, R. Variational Methods for the Solution of Problems of Equilibrium and 
Vibrations. Bulletin of the American Mathematical Society, v. 49, n. 1, p. 1-24, 1943. American Mathematical Society.

CRISFIELD, M. A. A consistent co-rotational formulation for non-linear, threedimensional, beam-elements. Computer Methods in Applied Mechanics and Engineering, v. 81, n. 2, p. 131-150, 1990. North-Holland.

CRISFIELD, M. A. Non-linear Finite Element Analysis of Solids and Structures: Essentials. 1991.

ESCAlONA, J. L.; HUSSiEN, H. A.; ShabanA, A. A. APPlication OF the ABSOLUTE NODAL CO-ORDINATE FORMULATION TO MULTIBODY SYSTEM DYNAMICS. Journal of Sound and Vibration, v. 214, n. 5, p. 833-851, 1998.

GARCÍA-VALLEJO, D.; ESCALONA, J. L.; MAYO, J.; DOMÍNGUEZ, J. Describing Rigid-Flexible Multibody Systems Using Absolute Coordinates. Nonlinear Dynamics, v. 34, p. 75-94, 2003.

GÉRADIN, M.; CARDONA, A. Flexible multibody dynamics: a finite element approach. 2001.

GOTO, Y.; YOSHIMITSU, T.; OBATA, M. Elliptic integral solutions of plane elastica with axial and shear deformations. International Journal of Solids and Structures, v. 26, n. 4, p. 375-390, 1990.

GRECO, M. Análise de problemas de contato/impacto em estruturas de comportamento não linear pelo método dos elementos finitos., 2004. Tese de doutorado, EESC-USP.

GRECO, M.; CODA, H. B. Positional FEM formulation for flexible multi-body dynamic analysis. Journal of Sound and Vibration, v. 290, n. 3-5, p. 1141-1174, 2006.

HARTOG, J. P. DEN. Mechanical Vibrations. 1985.

HOUSNER, G. W.; BERGMAN, L. A.; CAUGHEY, T. K.; et al. Structural control: 
Past, present, and future. Journal of Engineering Mechanics-Asce, v. 123, n. 9, p. 897-971, 1997. American Society of Civil Engineers.

JIANG, L.; CHERNUKA, M. W.; PEGG, N. G. A co-rotational, updated Lagrangian formulation for geometrically nonlinear finite element analysis of shell structures. Finite Elements in Analysis and Design, v. 18, n. 1-3, p. 129-140, 1994.

KANE, C.; MARSDEN, J. E.; ORTIZ, M.; WEST, M. Variational integrators and the Newmark algorithm for conservative and dissipative mechanical systems. International Journal for Numerical Methods in Engineering, v. 49, n. 10, p. 12951325, 2000.

KERR, C. N. LARGE DEFLECTIONS OF A SQUARE FRAME. The Quarterly Journal of Mechanics and Applied Mathematics, v. 17, n. 1, p. 23-38, 1964. Oxford University Press.

LEE, S.-H.; PARK, T.-W.; SEO, J.-H.; YOON, J.-W.; JUN, K.-J. The development of a sliding joint for very flexible multibody dynamics using absolute nodal coordinate formulation. Multibody System Dynamics, v. 20, n. 3, p. 223-237, 2008.

M. B. WONG AND F. TIN-LOIS. Geometrically Nonlinear Analysis of Elastic. , v. 34, n. 4, p. 633-640, 1990.

MACIEL, D. N. Análise de problemas elásticos não-lineares geométricos empregando o método dos elementos finitos posicional. , 2008. Departamento de Engenharia de Estruturas.

MADEIRA, R. H.; CODA, H. B.; MADEIRA, R. H.; CODA, H. B. Kelvin Viscoelasticity and Lagrange Multipliers Applied to the Simulation of Nonlinear Structural Vibration Control. Latin American Journal of Solids and Structures, v. 13, n. 5, p. 964-991, 2016. Associação Brasileira de Ciências Mecânicas.

MARQUES, G. C. DOS S. C. Estudo e desenvolvimento de código computacional 
baseado no MEF para análise dinâmica não linear geométrica de sólidos bidimensionais. , 2006. Departamento de Engenharia de Estruturas.

MATTIASSON, K. Numerical results from large deflection beam and frame problems analysed by means of elliptic integrals. International Journal for Numerical Methods in Engineering, v. 17, n. 1, p. 145-153, 1981.

MOKHA, A.; CONSTANTINOU, M. C.; REINHORN, A. M.; ZAYAS, V. A. Experimental Study of Friction-Pendulum Isolation System. Journal of Structural Engineering, v. 117, n. 4, p. 1201-1217, 1991.

NAGARAJAIAH, S.; REINHORN, A. M.; CONSTANTINOU, M. C. Nonlinear Dynamic Analysis of 3-D-Base-Isolated Structures. Journal of Structural Engineering, v. 117, n. 7, p. 2035-2054, 1991.

PACCOLA, R.; CODA, H. AcadView: Software para pós-processamento em elementos finitos 2D e 3D. , 2005. São Carlos: Escola de Engenharia de São Carlos, Universidade de São Paulo.

PASCON, J. P. Sobre modelos constitutivos não lineares para materiais com gradação funcional exibindo grandes deformações: implementação numérica em formulação não linear geométrica. , 2012. Departamento de Engenharia de Estruturas.

PAULTRE, P. Dynamics of structures. ISTE, 2010.

ROMERO, I. Formulation and performance of variational integrators for rotating bodies. Computational Mechanics, v. 42, n. 6, p. 825-836, 2008.

ROMERO, I.; ARMERO, F. An objective finite element approximation of the kinematics of geometrically exact rods and its use in the formulation of an energymomentum conserving scheme in dynamics. International Journal for Numerical Methods in Engineering, v. 54, n. 12, p. 1683-1716, 2002. 
SANCHES, R. A. K. Sobre o acoplamento fluido-casca utilizando o Método dos Elementos. Finitos. , 2011. Departamento de Engenharia de Estruturas.

SANCHES, R. A. K.; CODA, H. B. Unconstrained vector nonlinear dynamic shell formulation applied to Fluid Structure Interaction. Computer Methods in Applied Mechanics and Engineering, v. 259, p. 177-196, 2013.

SIMO, J. C.; TARNOW, N. The discrete energy-momentum method. Conserving algorithms for nonlinear elastodynamics. ZAMP Zeitschrift $f \bullet r$ angewandte Mathematik und Physik, v. 43, n. 5, p. 757-792, 1992.

SIQUEIRA, T. M. Análise dinâmica não linear geométrica de estruturas e mecanismos reticulados planos com ligações deslizantes. , 2016. Departamento de Engenharia de Estruturas.

SOONG, T.; DARGUSH, G. F. Passive energy dissipation systems in structural engineering by T. T. Soong and G. F. Dargush John Wiley \&amp; Sons Chichester, 1997 ISBN 0-471-96821-8. Wiley, 1997.

SUGIYAMA, H.; ESCALONA, J. L.; SHABANA, A. A. Formulation of ThreeDimensional Joint Constraints Using the Absolute Nodal Coordinates. Nonlinear Dynamics, v. 31, n. 2, p. 167-195, 2003. Kluwer Academic Publishers.

TURNER, M. J.; CLOUGH, R. W.; MARTIN, H. C.; TOPP, L. J. Stiffness and Deflection Analysis of Complex Structures. Journal of the Aeronautical Sciences (Institute of the Aeronautical Sciences), v. 23, n. 9, p. 805-823, 1956.

WARBURTON, G. B. Optimum absorber parameters for various combinations of response and excitation parameters. Earthquake Engineering \& Structural Dynamics, v. 10 , n. 3, p. 381-401, 1982. John Wiley \& Sons, Ltd.

YAO, J. T. P. Concept of Structural Control. , v. 98, n. 7, p. 1567-1574, 1972.

ZACCARIA, D.; BIGONI, D.; NOSELLI, G.; MISSERONI, D. Structures buckling 
under tensile dead load. Proceedings of the Royal Society of London A: Mathematical, Physical and Engineering Sciences, v. 467, n. 2130, 2011.

ZAYAS, V. A.; LOW, S. S.; MAHIN, S. A. A Simple Pendulum Technique for Achieving Seismic Isolation. Earthquake Spectra, v. 6, n. 2, p. 317-333, 1990. ZIENKIEWICZ, O. .; TAYLOR, R. . The Finite Element Method. 5 ed. ed. 1988. 